\title{
Direction Finding for Unmanned Aerial Systems Using Rhombic Antennas and Amplitude Comparison Monopulse
}

\author{
by \\ Ryan Kuiper
}

A thesis submitted to the Faculty of Graduate and

Postdoctoral Affairs in partial fulfillment of the requirements for the degree of

Master of Applied Science in

Electrical and Computer Engineering

Carleton University

Ottawa, Ontario

(C) 2017

Ryan Kuiper 


\section{Abstract:}

The purpose of this thesis is to design an antenna for a UAS (Unmanned Aerial System) comprised of an aircraft and a two channel transceiver/spectrum recording device for a DF (Direction Finding) application with an error of 6.12 degrees or less. The UAS had to work in a bandwidth from $0.6-6 \mathrm{GHz}$ and under the size constraints imposed by the bottom face of the transceiver/spectrum recording device $(19.4 \times 32.4 \mathrm{~cm})$. Due to the large operating spectrum of the DF UAS and size constraints imposed by the aircraft, a multi-antenna rhombic antenna solution is used.

The DF portion of the thesis is done using amplitude comparison monopulse with a 45 degree squint angle. Therefore, the rhombic antenna elements were designed to have a radiation pattern which allowed for this squint angle.

Once the requirements were accounted for and simulated, the rhombic antenna elements were built and tested with Carleton University's anechoic chamber. 


\section{Acknowledgments:}

I would like to thank my supervisor Dr. Jim Wight for helping me with my thesis topic and Carleton University for supporting me financially during my studies. 


\section{Table of Contents}

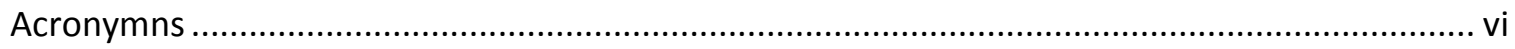

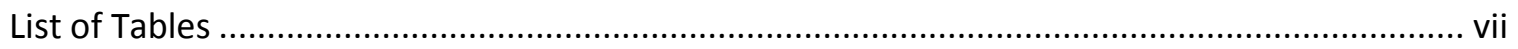

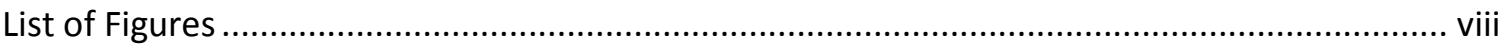

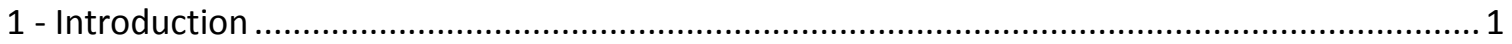

$1.1 \quad$ Problem Statement and Proposed Solution ......................................................... 1

1.2 UAS

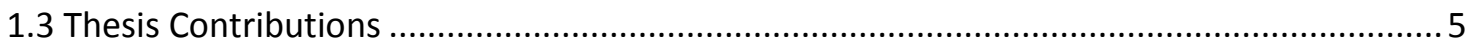

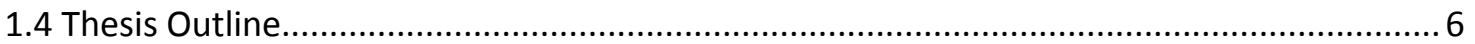

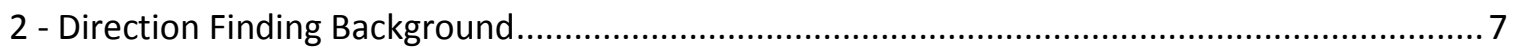

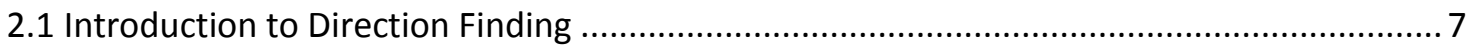

2.2 Types of Direction Finding in Two Channel Systems ........................................................ 8

2.2 Amplitude Comparison Monopulse Theory .................................................................... 9

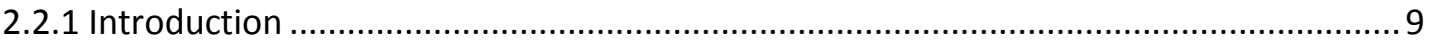

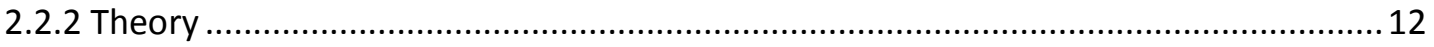

2.2.3 Comparing Amplitude Comparison Monopulse to Other Monopulse Methods .......... 19

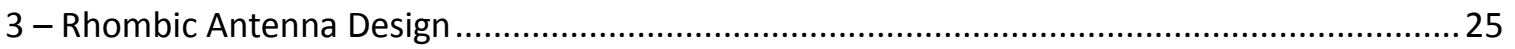

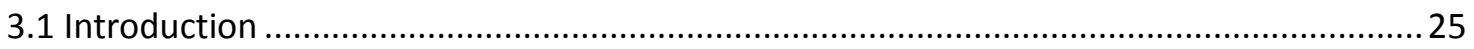

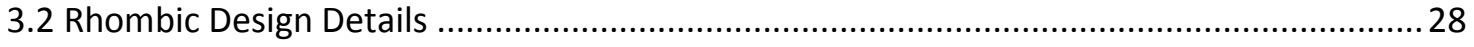

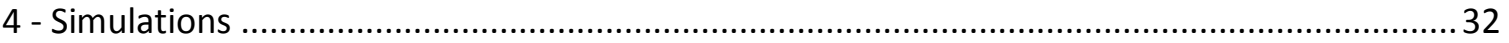

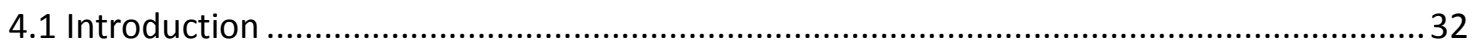

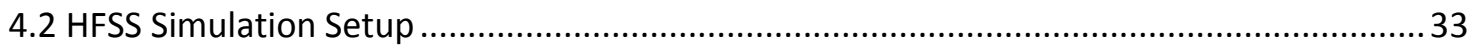

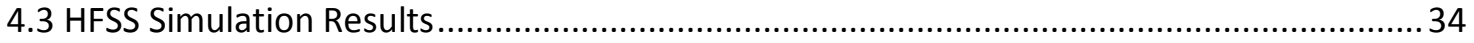

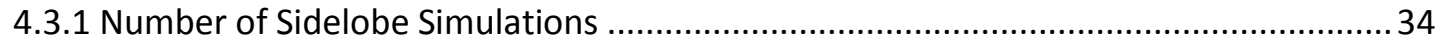

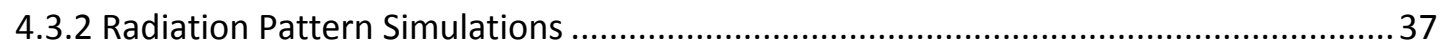

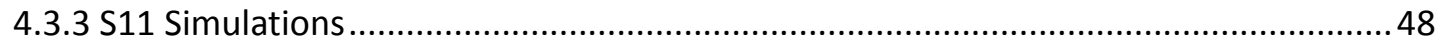

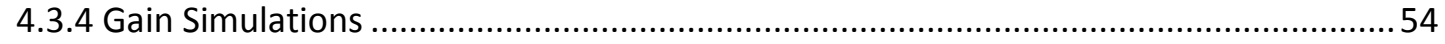

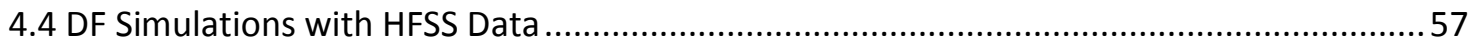

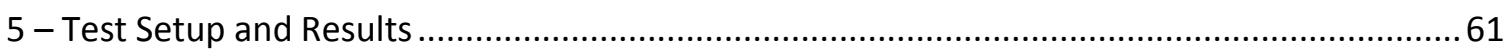

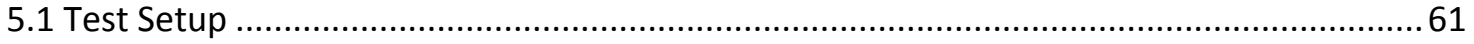

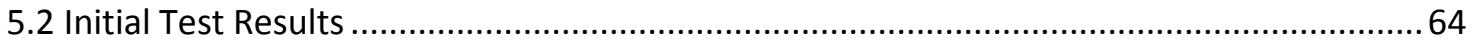


5.2.1 Upper Band Test Results

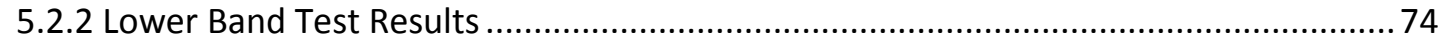

5.3 DF Simulations with Assembled Rhombic Measurements ................................................. 83

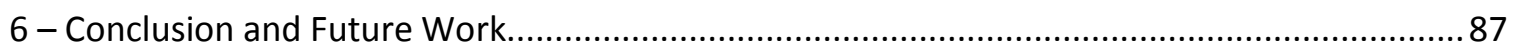

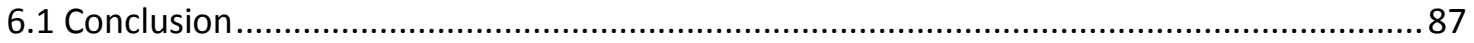

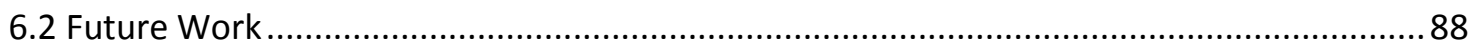

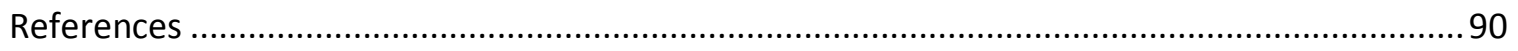

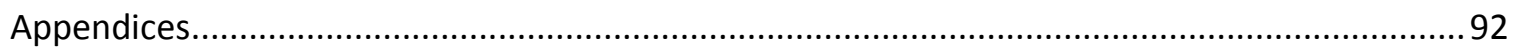

Appendix A: Lower Frequency Band ～Radiation Pattern Simulations ...................................92

Appendix B: Upper Frequency Band Radiation Pattern Simulations ...................................97

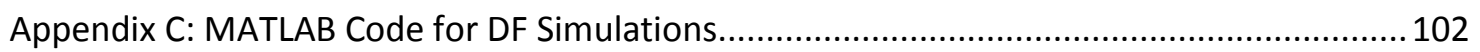




\section{Acronymns}

Add White Gaussian Noise (AWGN)

Amplitude Modulation (AM)

Antenna Under Test (AUT)

Automatic Gain Control (AGC)

Bandwidth (BW)

Comma-Separated Values (CSV)

Direction Finding (DF)

Frequency Modulation (FM)

Half-Power Beamwidth (HPBW)

High Frequency (HF)

High Frequency Structure Simulator (HFSS)

Matrix Laboratory (MATLAB)

Perfectly Matched Layer (PML)

Phase Modulation (PM)

Radio Frequency (RF)

Signal to Noise Ratio (SNR)

SubMiniature Version A (SMA)

Ultra High Frequency (UHF)

Unmanned Aerial System (UAS)

Unmanned Aerial Vehicle (UAV)

Very High Frequency (VHF) 


\section{List of Tables}

Table 1: Amplitude comparison monopulse bearing error by system type [10]

Table 2: Effect of SNR on monopulse error signal at $800 \mathrm{MHz}$ for a

12.8

$\mathrm{cm}$ leg length rhombic

Table 3: Effect of UAV height on monopulse error signal at $800 \mathrm{MHz}$ for a

$12.8 \mathrm{~cm}$ leg length rhombic 60

Table 4: Monopulse Error Results from -15 to $15^{\circ}$ rhombic offsets in the azimuth plane........... 86 


\section{List of Figures}

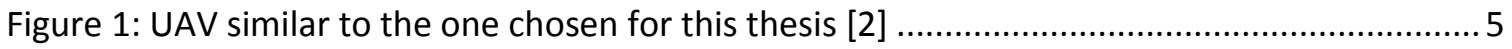

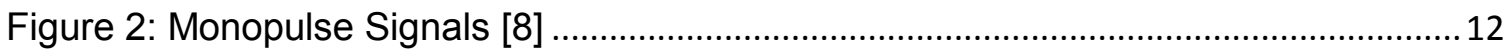

Figure 3: Amplitude comparison monopulse example [9] ...................................................... 14

Figure 4: Zoomed in error signal (Error signal voltage vs Azimuth angle in ${ }^{\circ}$ )............................... 16

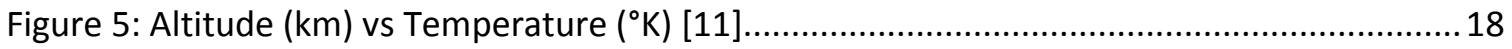

Figure 6: RMS angular error vs target angle-off-boresight for changing SNR [10] ......................21

Figure 7: Block diagram of a monopulse hardware based system [10] ....................................... 22

Figure 8: Open loop vs closed loop monopulse system block diagrams ......................................23

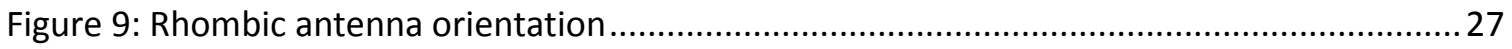

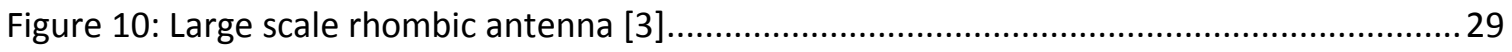

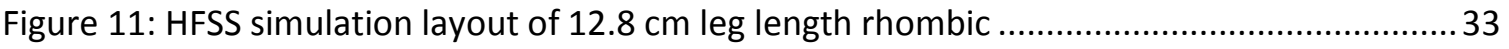

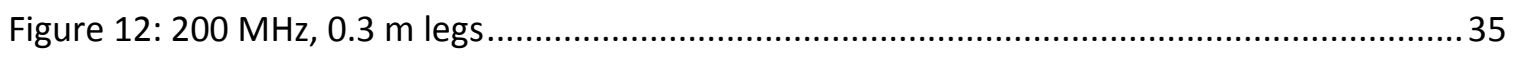

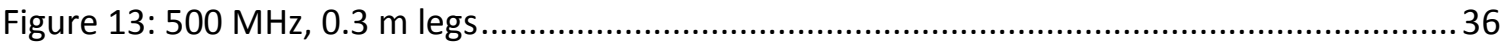

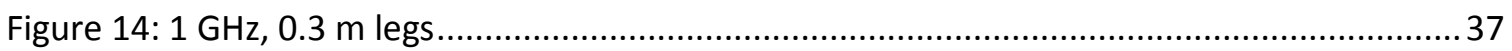

Figure 15: $5 \mathrm{~mm}$ leg length at $6 \mathrm{GHz}$ with $2 \mathrm{~mm}$ wire thickness and $1 \mathrm{~mm}$ separation .................38

Figure 16: $5 \mathrm{~mm}$ leg length at $5 \mathrm{GHz}$ with $2 \mathrm{~mm}$ wire thickness and $1 \mathrm{~mm}$ separation ................39

Figure 17: $1.2 \mathrm{~cm}$ leg length at $3.5 \mathrm{GHz}$ with $2 \mathrm{~mm}$ wire thickness and $1 \mathrm{~mm}$ separation ........... 40

Figure 18: $1.8 \mathrm{~cm}$ leg length at $3.5 \mathrm{GHz}$ with $2 \mathrm{~mm}$ wire thickness and $1 \mathrm{~mm}$ separation ........... 41

Figure 19: $2.2 \mathrm{~cm}$ leg length at $3.5 \mathrm{GHz}$ with $2 \mathrm{~mm}$ wire thickness and $1 \mathrm{~mm}$ separation ........... 42

Figure 20: $2.5 \mathrm{~cm}$ leg length at $3.5 \mathrm{GHz}$ with $2 \mathrm{~mm}$ wire thickness and $1 \mathrm{~mm}$ separation ........... 43

Figure 21: $2.3 \mathrm{~cm}$ leg length at $3.5 \mathrm{GHz}$ with $2 \mathrm{~mm}$ wire thickness and $1 \mathrm{~mm}$ separation ........... 44

Figure 22: $12.8 \mathrm{~cm}$ leg length at $800 \mathrm{MHz}$ with $2 \mathrm{~mm}$ wire thickness and $1 \mathrm{~mm}$ separation........ 45

Figure 23: $12.5 \mathrm{~cm}$ leg length at $800 \mathrm{MHz}$ with $2 \mathrm{~mm}$ wire thickness and $1 \mathrm{~mm}$ separation........ 46

Figure 24: $12.7 \mathrm{~cm}$ leg length at $800 \mathrm{MHz}$ with $2 \mathrm{~mm}$ wire thickness and $1 \mathrm{~mm}$ separation........ 47

Figure 25: S11 for $12.8 \mathrm{~cm}$ leg length rhombic with a $600 \Omega$ terminating resistance ................... 48

Figure 26: $\mathrm{S} 11$ for $12.8 \mathrm{~cm}$ leg length rhombic with a $700 \Omega$ terminating resistance ................... 49

Figure 27: S11 for $12.8 \mathrm{~cm}$ leg length rhombic with a $500 \Omega$ terminating resistance .................... 49

Figure 28: S11 for $2.3 \mathrm{~cm}$ leg length rhombic with a $600 \Omega$ terminating resistance ......................50

Figure 29: S11 for $2.3 \mathrm{~cm}$ leg length rhombic with a $50 \Omega$ terminating resistance ........................5 51

Figure 30: S11 for $2.3 \mathrm{~cm}$ leg length rhombic with a $200 \Omega$ terminating resistance .....................51

Figure 31: S11 for $2.3 \mathrm{~cm}$ leg length rhombic with a $100 \Omega$ terminating resistance .....................5 52

Figure 32: S11 for $6 \mathrm{~cm}$ leg length rhombic with a $600 \Omega$ terminating resistance ........................53

Figure 33: $6 \mathrm{~cm}$ leg length rhombic gain with a $600 \Omega$ terminating resistance ..............................55

Figure $34: 2.3 \mathrm{~cm}$ leg length rhombic gain with a $100 \Omega$ terminating resistance ..........................55

Figure $35: 2.5 \mathrm{~cm}$ leg length rhombic gain with a $100 \Omega$ terminating resistance ...........................56

Figure 36: $2 \mathrm{~cm}$ leg length rhombic gain with a $100 \Omega$ terminating resistance ............................56

Figure 37: Magnitude of monopulse error signal vs phi with no noise or path loss .....................58

Figure 38: Zoomed magnitude of monopulse error signal vs phi with no noise or path loss ....... 59

Figure 39: Anechoic chamber with transmit and receive antennas ........................................... 62 
Figure 40: Carleton University's anechoic chamber lab. The spectrum analyzer is on the left and the turntable controller is on the bottom right 63

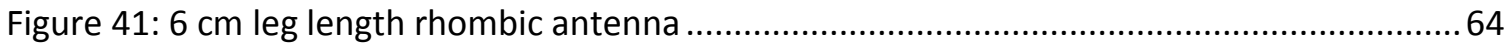

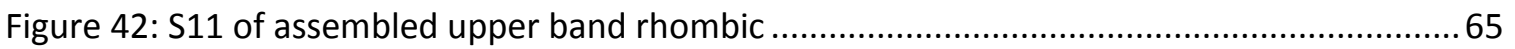

Figure 43: $2 \mathrm{GHz}$ S21 (dB) in azimuth plane for $2 \mathrm{~cm}$ leg length rhombic ..................................6 66

Figure 44: $2.5 \mathrm{GHz}$ S21 (dB) in azimuth plane for $2 \mathrm{~cm}$ leg length rhombic ................................ 67

Figure 45: $3 \mathrm{GHz}$ S21 (dB) in azimuth plane for $2 \mathrm{~cm}$ leg length rhombic .................................67

Figure 46: $3.5 \mathrm{GHz}$ S21 (dB) in azimuth plane for $2 \mathrm{~cm}$ leg length rhombic ..............................68

Figure 47: $4 \mathrm{GHz}$ S21 (dB) in azimuth plane for $2 \mathrm{~cm}$ leg length rhombic .................................. 68

Figure 48: $4.5 \mathrm{GHz}$ S21 (dB) in azimuth plane for $2 \mathrm{~cm}$ leg length rhombic ................................69

Figure 49: $5 \mathrm{GHz}$ S21 (dB) in azimuth plane for $2 \mathrm{~cm}$ leg length rhombic .................................69

Figure 50: $5.5 \mathrm{GHz}$ S21 (dB) in azimuth plane for $2 \mathrm{~cm}$ leg length rhombic ............................... 70

Figure 51: $6 \mathrm{GHz}$ S21 (dB) in azimuth plane for $2 \mathrm{~cm}$ leg length rhombic .................................. 70

Figure 52: Gain of the EM6961 gain standard vs frequency [17] ........................................... 72

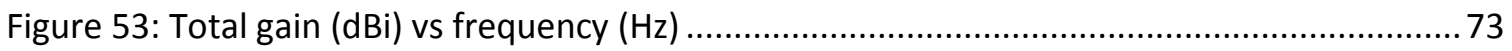

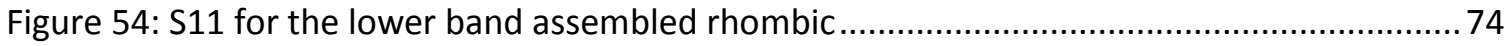

Figure 55: $0.6 \mathrm{GHz}$ S21 (dB) in azimuth plane for $6 \mathrm{~cm}$ leg length rhombic ................................ 75

Figure 56: $0.8 \mathrm{GHz}$ S21 (dB) in azimuth plane for $6 \mathrm{~cm}$ leg length rhombic ................................ 76

Figure 57: $1 \mathrm{GHz}$ S21 (dB) in azimuth plane for $6 \mathrm{~cm}$ leg length rhombic ..................................77

Figure 58: $1.2 \mathrm{GHz}$ S21 (dB) in azimuth plane for $6 \mathrm{~cm}$ leg length rhombic ................................ 78

Figure 59: $1.4 \mathrm{GHz}$ S21 $(\mathrm{dB})$ in azimuth plane for $6 \mathrm{~cm}$ leg length rhombic .............................. 79

Figure $60: 1.6 \mathrm{GHz}$ S21 (dB) in azimuth plane for $6 \mathrm{~cm}$ leg length rhombic ............................... 80

Figure $61: 1.8 \mathrm{GHz}$ S21 (dB) in azimuth plane for $6 \mathrm{~cm}$ leg length rhombic .............................. 81

Figure $62: 2 \mathrm{GHz}$ S21 (dB) in azimuth plane for $6 \mathrm{~cm}$ leg length rhombic .................................. 82

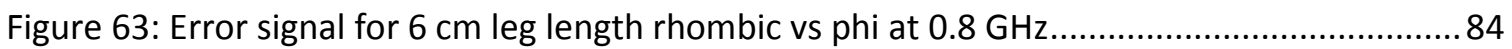

Figure 64: Zoomed in error signal of $6 \mathrm{~cm}$ leg length rhombic vs phi at $0.8 \mathrm{GHz} \ldots \ldots \ldots \ldots \ldots \ldots \ldots \ldots . . . . . . . . . . . . .85$

Figure 65: $6 \mathrm{~cm}$ leg length at $0.6 \mathrm{GHz}$ with $2 \mathrm{~mm}$ wire thickness and $1 \mathrm{~mm}$ separation...............92

Figure $66: 6 \mathrm{~cm}$ leg length at $0.8 \mathrm{GHz}$ with $2 \mathrm{~mm}$ wire thickness and $1 \mathrm{~mm}$ separation...............93

Figure 67: $6 \mathrm{~cm}$ leg length at $1 \mathrm{GHz}$ with $2 \mathrm{~mm}$ wire thickness and $1 \mathrm{~mm}$ separation..................93

Figure $68: 6 \mathrm{~cm}$ leg length at $1.2 \mathrm{GHz}$ with $2 \mathrm{~mm}$ wire thickness and $1 \mathrm{~mm}$ separation...............94

Figure 69: $6 \mathrm{~cm}$ leg length at $1.4 \mathrm{GHz}$ with $2 \mathrm{~mm}$ wire thickness and $1 \mathrm{~mm}$ separation...............94

Figure 70: $6 \mathrm{~cm}$ leg length at $1.6 \mathrm{GHz}$ with $2 \mathrm{~mm}$ wire thickness and $1 \mathrm{~mm}$ separation...............95

Figure $71: 6 \mathrm{~cm}$ leg length at $1.8 \mathrm{GHz}$ with $2 \mathrm{~mm}$ wire thickness and $1 \mathrm{~mm}$ separation...............95

Figure $72: 6 \mathrm{~cm}$ leg length at $2 \mathrm{GHz}$ with $2 \mathrm{~mm}$ wire thickness and $1 \mathrm{~mm}$ separation.................96

Figure $73: 2 \mathrm{~cm}$ leg length at $2 \mathrm{GHz}$ with $2 \mathrm{~mm}$ wire thickness and $1 \mathrm{~mm}$ separation..................97

Figure 74: $2 \mathrm{~cm}$ leg length at $2.5 \mathrm{GHz}$ with $2 \mathrm{~mm}$ wire thickness and $1 \mathrm{~mm}$ separation...............98

Figure 75: $2 \mathrm{~cm}$ leg length at $3 \mathrm{GHz}$ with $2 \mathrm{~mm}$ wire thickness and $1 \mathrm{~mm}$ separation..................98

Figure $76: 2 \mathrm{~cm}$ leg length at $3.5 \mathrm{GHz}$ with $2 \mathrm{~mm}$ wire thickness and $1 \mathrm{~mm}$ separation..............99

Figure 77: $2 \mathrm{~cm}$ leg length at $4 \mathrm{GHz}$ with $2 \mathrm{~mm}$ wire thickness and $1 \mathrm{~mm}$ separation.................99

Figure $78: 2 \mathrm{~cm}$ leg length at $4.5 \mathrm{GHz}$ with $2 \mathrm{~mm}$ wire thickness and $1 \mathrm{~mm}$ separation............. 100

Figure 79: $2 \mathrm{~cm}$ leg length at $5 \mathrm{GHz}$ with $2 \mathrm{~mm}$ wire thickness and $1 \mathrm{~mm}$ separation................ 100

Figure 80: $2 \mathrm{~cm}$ leg length at $5.5 \mathrm{GHz}$ with $2 \mathrm{~mm}$ wire thickness and $1 \mathrm{~mm}$ separation............. 101 
Figure $81: 2 \mathrm{~cm}$ leg length at $6 \mathrm{GHz}$ with $2 \mathrm{~mm}$ wire thickness and $1 \mathrm{~mm}$ separation........ 


\section{1 - Introduction}

\subsection{Problem Statement and Proposed Solution}

In the scope of electronic warfare there have been several large-scale improvements over time. This type of warfare started in the $20^{\text {th }}$ century when a need for long-range communications and tracking vehicles arose during war. These requirements led to the development of what are now staples of electronic warfare in radar, FM/AM/PM (Frequency Modulation/Amplitude Modulation/Phase Modulation) communications, jamming, and spoofing. Now that most countries have either become experts in these topics, or have started to get involved with them there is a need for something new that can combat them effectively.

With the recent rise of drone based technologies a solution to this problem can be found. The signals that an adversary is sending can be recorded and played back at a later time using a UAV (Unmanned Aerial Vehicle) carrying a receiver and a recording device. Using a spectrum recording device allows the DF algorithm to be run after a UAS's flight path in post-processing. With this existing technology, a DF technique could be implemented which could find the direction and frequency at which a signal is transmitted. An antenna could be developed which can receive signals to interface with a DF algorithm for this type of UAS.

There are known theoretical techniques to find both what a signal's characteristics are and where the signal's source is located. This is incredibly useful in electronic warfare. One side of a conflict could take control of the usable spectrum by flying a drone over an area of interest, finding where their adversary's transmitters are located and neutralizing 
them before an organized attack. This could lead to a large reduction in lives lost for the side who chooses to implement this technology.

Some classified techniques have been implemented which have achieved DF results with an approximate error of 10 degrees in a UAS implementation. This is done without the use of spectrum recording devices or post-processing DF. The error for this implementation is far too high to be practical in a real world UAS implementation. This thesis aims to improve this DF error to be better than 6.12 degrees.

The equipment necessary to record the RF (Radio Frequency) spectrum from $0.6-6 \mathrm{GHz}$ for this application exists. What is needed is a suitable DF algorithm with an antenna which can minimize the error of that algorithm in a UAS implementation. This thesis will focus on solving the issues related to DF for this system making use of existing antennas and a novel DF approach which occupies a small footprint. The caveat to this system is that the antennas need to be mounted at the bottom of a small UAS in a small area. Once a suitable drone was found, it was determined that the space available for the DF antennas would be approximately 19.4 x $32.4 \mathrm{~cm}$. Most conventional techniques for DF and antenna systems are not usable because of this size restriction.

Using the concept of a UAS for DF, several advantages can be gained. For example, since UAS's fly several thousand feet in the air (up to about 10000), multipath will be greatly reduced or eliminated. Without multipath there are amplitude based methods used in radar which can find the direction of a transmitter with less error. For this reason, a tracking radar algorithm called open loop amplitude comparison monopulse is chosen as the DF technique for this thesis. 
Other advantages that can be gained in a UAS with post-processing capabilities for amplitude based DF methods include higher SNR (Signal to Noise Ratio) due to a need for less equipment, lower equipment temperatures than normal (since the UAS will be in the upper portion of the troposphere) [1], and half the path loss. Less equipment is needed in a post-processing DF UAS than a tracking radar since amplitude comparison monopulse in a tracking radar requires circuitry to find the direction of a radar target in real time, whereas this UAS does not. This, in combination with the lower operating temperature of the equipment on the UAS will result in better noise conditions than amplitude comparison monopulse would have in a radar application. There is also half the path loss of a radar system since a radar system must transmit and receive a signal and this DF UAS is only receiving. Chapters 2 and 4 of this thesis will show why this is the case and illustrate the ramifications of lower noise in an amplitude comparison monopulse system.

Once the DF method was determined, a suitable antenna was chosen for it. Due to the size constraints of the UAS chosen, the desired radiation pattern characteristics, and broad spectrum of this application rhombic antennas were chosen. Rhombic antennas provide good radiation pattern characteristics for amplitude comparison monopulse since they concentrate a large portion of their radiation pattern into the main lobe. The remainder of the power is largely concentrated in the back lobe as rhombics can be designed to have no sidelobes. These rhombic characteristics will be shown in chapters 3 and 4 of this thesis. 


\subsection{UAS}

A UAS is a UAV with some components added which allow the UAV to perform a task it otherwise would not be able to. In the case of this thesis, the UAS allows its operator to both receive and record signals of interest from $0.6-6 \mathrm{GHz}$. DF techniques are used in post-processing to find where the signal came from.

The type of aircraft that is selected for this thesis is a UAV which has propellers similar to a helicopter. This helps reduce the aircraft jitter which in turn improves the DF capabilities of the UAS because it can be assumed that the UAV does not tilt in the elevation plane. If the UAV does not tilt, it can then be assumed that the antenna is always looking horizontally at an area or signal of interest. Figure 1: UAV similar to the one chosen for this thesis [2] shows a UAV similar to the one that is used for this thesis. Note that a spectrum recording device and a receiver would be suspended below the UAV as a payload. The rhombic antennas would be connected below the equipment. 


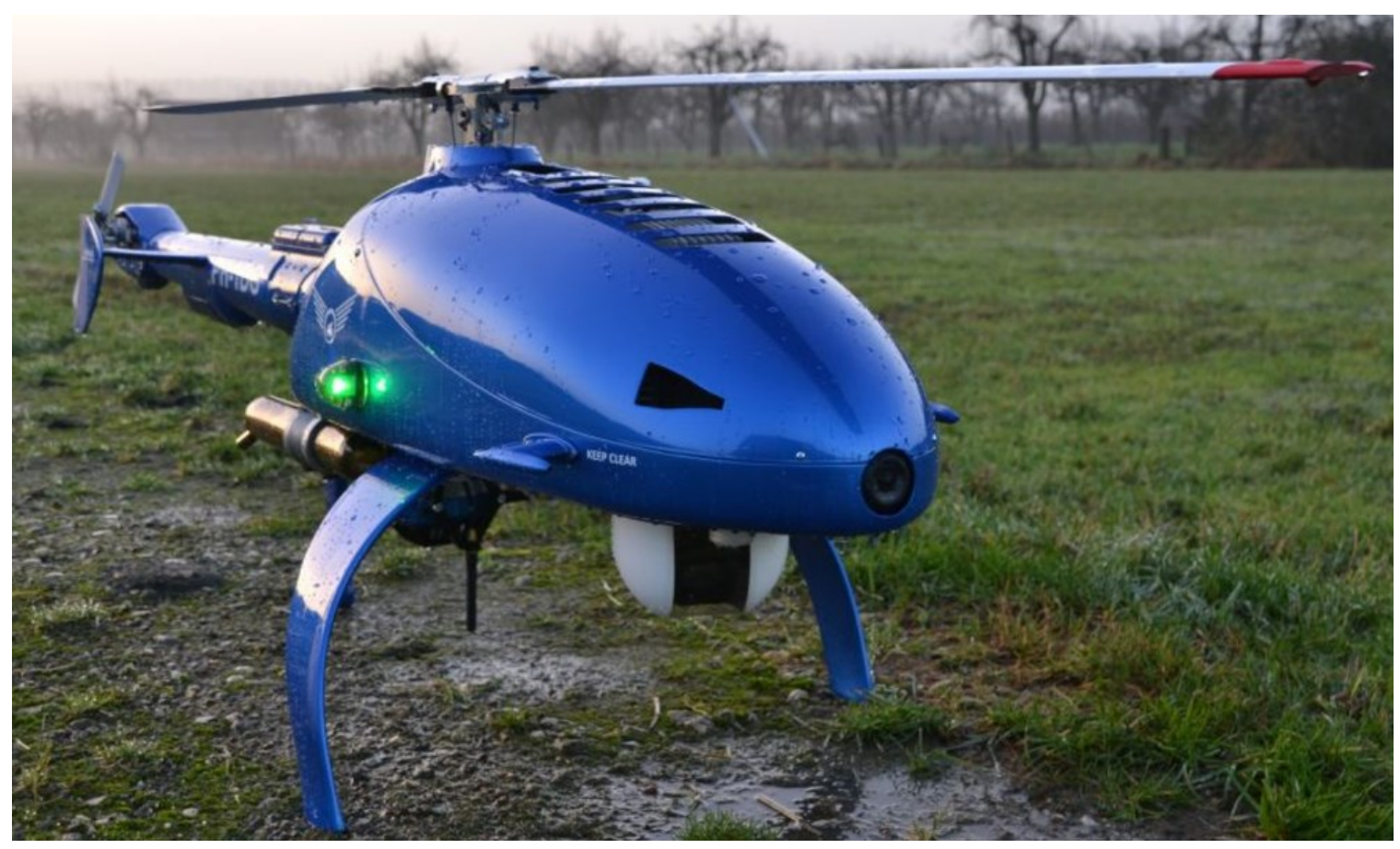

Figure 1: UAV similar to the one chosen for this thesis [2]

\subsection{Thesis Contributions}

The goal of this thesis is to design a DF system for a $0.6-6 \mathrm{GHz}$ UAS. According to the requirements of this UAS, the DF method must provide an error less than 6.12 degrees.

If a DF system could be made with an error far below 10 degrees for a UAS it could revolutionize the way spectral warfare is done. This low error UAS DF system could be used to find the sources of a jamming or interfering signal in a warzone.

This thesis offers a DF solution from $0.6-6 \mathrm{GHz}$ assuming a two channel transceiver/spectrum recorder with a reasonable gain. A DF system has not previously been implemented with open-loop amplitude comparison monopulse. The DF signal processing algorithms are done in MATLAB (Matrix Laboratory).

Rhombic antennas were used as the receiving antennas for this thesis. Prototypes of these antennas are developed and tested using Carleton University's anechoic chamber. 
Rhombics were used in the HF/VHF (High Frequency/Very High Frequency) bands [1], but are used in a more broad $0.6-6 \mathrm{GHz}$ spectrum in this thesis. They are also being used as a DF antenna on a UAS, both of which have not been done before.

A spectral surveillance UAS which records spectrum and utilizes DF to find the location of a transmitter of interest currently does not exist. UAS's which perform a DF algorithm have been implemented, however they have not been implemented with equipment which is capable of recording spectrum. This allows for some post-processing to be performed on the spectrum to gather additional information about a transmitted signal and reduce the SNR (Signal to Noise Ratio) of the system.

\subsection{Thesis Outline}

This thesis is broken down into 5 chapters after the first introduction chapter. Chapter 2 introduces DF, some methods used in DF implementations, and provides an in-depth analysis of the DF method used in this thesis as well as reasons why it is used instead of other methods. Chapter 3 introduces rhombic antennas and describes how they are designed. In Chapter 4, simulations were performed to determine what the ideal rhombic antenna size would be given the constraints of this thesis. In addition to this, DF simulations were performed using the ideal rhombic's radiation pattern. Chapter 5 covers the rhombic test equipment, test results, and DF simulation results using those test results. The final chapter (Chapter 6) concludes this thesis. 


\section{2 - Direction Finding Background}

\subsection{Introduction to Direction Finding}

DF is a method used to find where a signal of interest is coming from. A DF system contains a receiver, receiving antenna(s), and an algorithm which can be implemented using hardware or software. Uses for this type of technology include spectral warfare, search and rescue, etc. A DF system will estimate the direction of a transmitting element by measuring and performing an algorithm on the received signal.

Errors can be introduced to a DF system due to interference, sources of multipath at a receiver, and others depending on the type of DF algorithm that is used. In the case of this thesis, these DF system errors are insignificant since the DF is done with a UAS. This eliminates multipath and greatly reduces the likelihood of having to deal with any interference. Multipath is eliminated since the UAS is flown thousands of feet above the ground. Since the UAS is flown at this height, it also reduces the chances of interference since several transmitters that could cause interference will not be radiating upwards.

In the general case of using DF at ground level, finding the direction of an emitter in the azimuth direction is usually sufficient. However, in the case of this thesis, the elevation direction becomes important as well since the signals of interest are being received by a UAS. If a UAS is thousands of feet in the air, the elevation angle will matter far more than it would on the ground since the UAS needs to know if the transmitter is directly below it, or at some offset from the elevation axis. However, for the sake of a proof of concept of the accuracy of the DF technique used (and limitations of the test equipment used), the elevation angle is not addressed. 


\subsection{Types of Direction Finding in Two Channel Systems}

There are several types of DF that are used today. DF can be done with a single channel or multiple channels. When more channels are used, DF in general becomes more accurate. Since this thesis uses a 2 channel UAS, this section will focus on DF methods that are valid when using 2 channels. Some of these DF methods include polarization based DF, Doppler based DF, and monopulse DF.

Polarization based DF systems are implemented with dipole or loop antennas. They are used in applications in the HF band with small antennas. One of the first DF systems called the rotating-loop direction finder is a polarization based DF system. The system works by rotating the loop until a minimum received signal is found such that the incident wave is perpendicular to the antennas. The Watson-Watt method is then used to find the bearing of a target. This method in a two (co-located) antenna system takes the vector difference of the received signal from each antenna. The vector difference is then used to produce north-south and east-west signals. A bearing angle is then computed by taking the arctangent of the ratio of the north-south and east-west signals [4].

Doppler based DF systems are typically used in the VHF and UHF (Ultra High Frequency) bands for air traffic control. These systems are sensitive and are not greatly affected by multipath. They take a fairly long time relative to other methods in order to obtain a transmitter's direction. This is because a full scanning cycle of the systems antenna is needed to obtain a valid measurement [4]. A scanning cycle can take several milliseconds depending on the rotation speed of the antenna.

Monopulse based tracking radar systems can be implemented by measuring the phase or the amplitude of a received signal. Measurements can be taken using one set of sampled 
data (or one pulse). More samples can be used to improve the accuracy of the bearing measurement.

Amplitude comparison monopulse was chosen as the DF technique for this thesis. This is because polarization and Doppler DF systems are not suitable for a UAS implementation. Polarization DF systems are not suitable since the UAV has a propeller and no room for a servo system that would allow the antennas to properly rotate. Doppler DF systems use a more complex and time intensive algorithm to determine a transmitters bearing. A main advantage of using a Doppler DF system is that it is fairly resistant to multipath (which is not an issue in this thesis) which makes other DF methods more accurate in comparison to the Doppler method than they otherwise would be. The following section will describe why amplitude comparison monopulse was used instead of phase comparison monopulse. It will also provide a more detailed description of how amplitude comparison monopulse works.

\subsection{Amplitude Comparison Monopulse Theory}

\subsubsection{Introduction}

Monopulse, by definition is a technique that allows for making an angular measurement in two coordinates (elevation and azimuth) based on information from one pulse [5]. Hence, the name monopulse, where 'mono' means one and 'monopulse' means one pulse. This one pulse angular measurement can be performed using the phase and/or the amplitude characteristics of a received signal on multiple channels. While measurements can be performed on one pulse, angular measurements can be improved upon by taking additional measurements and taking the average result to increase the SNR and accuracy [5]. 
It is important to note that monopulse techniques are typically used in tracking radars and have not been used as a means of DF. Monopulse tracking radar systems would have a transmitter and a receiver such that a pulse could be sent towards a target and the reflection off of the target could be received. In tracking radar systems the algorithm is done in real-time with a set of circuits which calculate the error in the bearing of the received signal and try to minimize the error. However, with this thesis' application there is only a receiver with a passive DF algorithm which is done in post-processing.

Amplitude comparison monopulse is different from common modern DF techniques which rely on analyzing the phase of an incoming signal. Instead, amplitude comparison monopulse makes use of the amplitude of an incoming signal over two channels and the radiation pattern of the antennas that are receiving a signal. This is done by squinting the channels received signals and taking the ratio of the difference and summation signals. The difference and summation signals are generated by taking a point by point difference and summation of the two channels.

Note that amplitude or phase comparison monopulse analysis can be performed in either a closed loop or open loop configuration. In a closed-loop configuration, a servo uses a ratio (covered in the theory section) called an error signal. It then attempts to null the perceived error by moving the beam axis towards a desired target until its boresight is pointed towards the target. In an open-loop configuration, the direction the beam points in is assumed to be constant in response to the indicated off-axis angle. For a closed loop system, the target's direction is then found by finding the point at which the monopulse ratio is 0 . In an open loop system, the error signal is 0 when the angle where the $-3 \mathrm{~dB}$ points of the two receiving antennas intersect [6]. 
When using amplitude comparison monopulse as a means of DF, at least two received signals on two different channels must be used. The antennas connected to the two channels should be squinted such that the $-3 \mathrm{~dB}$ point of the two radiation patterns intersect. Since the $-3 \mathrm{~dB}$ points must be known, the radiation pattern of the receiving antennas must be well defined. Therefore, a significant disadvantage of amplitude comparison monopulse comes from multipath. However, since the antennas are to be mounted on a UAV, the effects of multipath will be minimal to non-existent depending on the operating height of the UAV and its surrounding environment.

It is important for amplitude comparison monopulse to use known antenna patterns for each frequency that it operates at. This means that if amplitude comparison monopulse is used as a DF technique, a lookup table is needed to determine the point of the radiation pattern the signal is received at.

Since multiple signals are received on separate channels at the same time with amplitude comparison monopulse, it uses a technique called simultaneous lobing. Simultaneous lobing receives signals from two partially overlapping antenna lobes at the same time. This helps prevent DF errors that are caused due to fluctuations in the received signal strength.

Important advantages of monopulse include a reduced vulnerability to jamming when compared to other tracking methods, better measurement efficiency, and reduced effects of target scintillation [7]. Target scintillation is reduced and measurement efficiency is increased since multiple measurements can be gathered from multiple channels using sequential lobing over several pulses or measurements. 


\subsubsection{Theory}

When two received signals (one signal received by two separate channels) overlap, the relative amplitude of the two signals can be used to determine the direction of the source of a transmitted signal. Either in real time or in post processing, the two signals acquired by receiving antennas are broken down into three other signals. One of which is the summation of the two signals, another is the difference between the two signals, and the last is the ratio of the sum and difference signals. From the ratio of the two signals, the direction of arrival of the transmitted signal can be found. All of these signals are shown in Figure 2: Monopulse Signals [8] below:
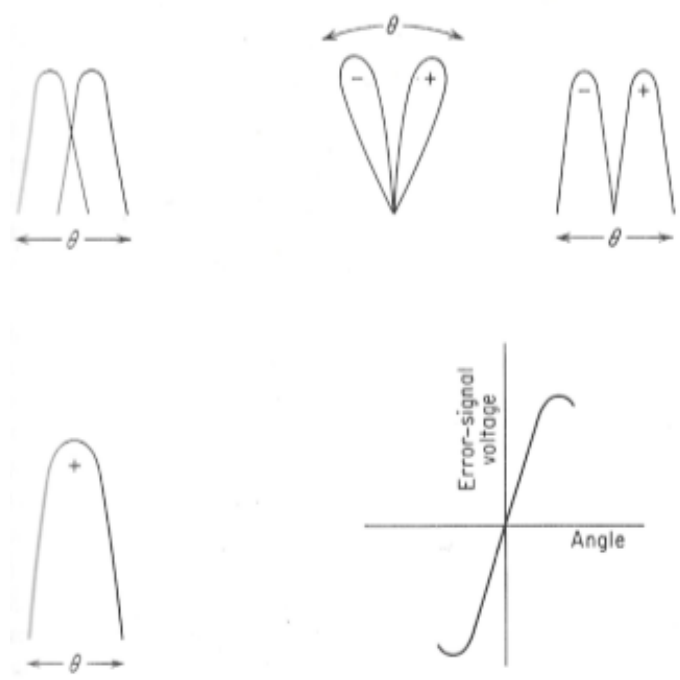

Figure 2: Monopulse Signals [8]

In Figure 2: Monopulse Signals [8], the top-left shows two overlapping antenna patterns. The bottom-left portion shows a sum pattern and the top-right portions show difference patterns. The bottom-right graph shows the error signal vs the azimuth or elevation angle. Note that the error signal is the ratio of the difference and summation signals. 
The overlapping antenna patterns have main lobes pointing in different directions. The difference in the boresight angle of the two main lobes are said to be squinted. This difference in boresight angle is called the squint angle [5]. In this thesis, the minimium squint angle over the frequency range of $0.6-6 \mathrm{GHz}$ must be at least 45 degrees since there is an elemental offset of 45 degrees between antenna elements. The 45 degree squint number is used since it is a convenient squint angle for a UAS which turns to get 360 degree azimuth coverage. However, in a typical open or closed loop monopulse system, the optimum squint angle is found using the following equation [5]:

$$
\theta_{q}=0.31 * \theta_{B}
$$

Where $\theta_{B}$ is the HPBW (Half Power Beamwidth) and $\theta_{q}$ is the optimum squint angle. A different optimum squint angle of $0.46 \theta_{B}$ is given by Rhodes and Sherman [5]. The 0.31 number corresponds to a beam crossover $1.2 \mathrm{~dB}$ down from the peak, while the 0.46 number corresponds to a beam crossover $2.6 \mathrm{~dB}$ down from the peak. In chapter 4 , it will be shown that the HPBW of the rhombic antennas is usually over 90 degrees and when multiplied by 0.46 or 0.31 often yields an optimum squint angle of 45 degrees or slightly less.

Figure 3: Amplitude comparison monopulse example [9] below shows the squint angle in relation to the received beam patterns is shown below: 


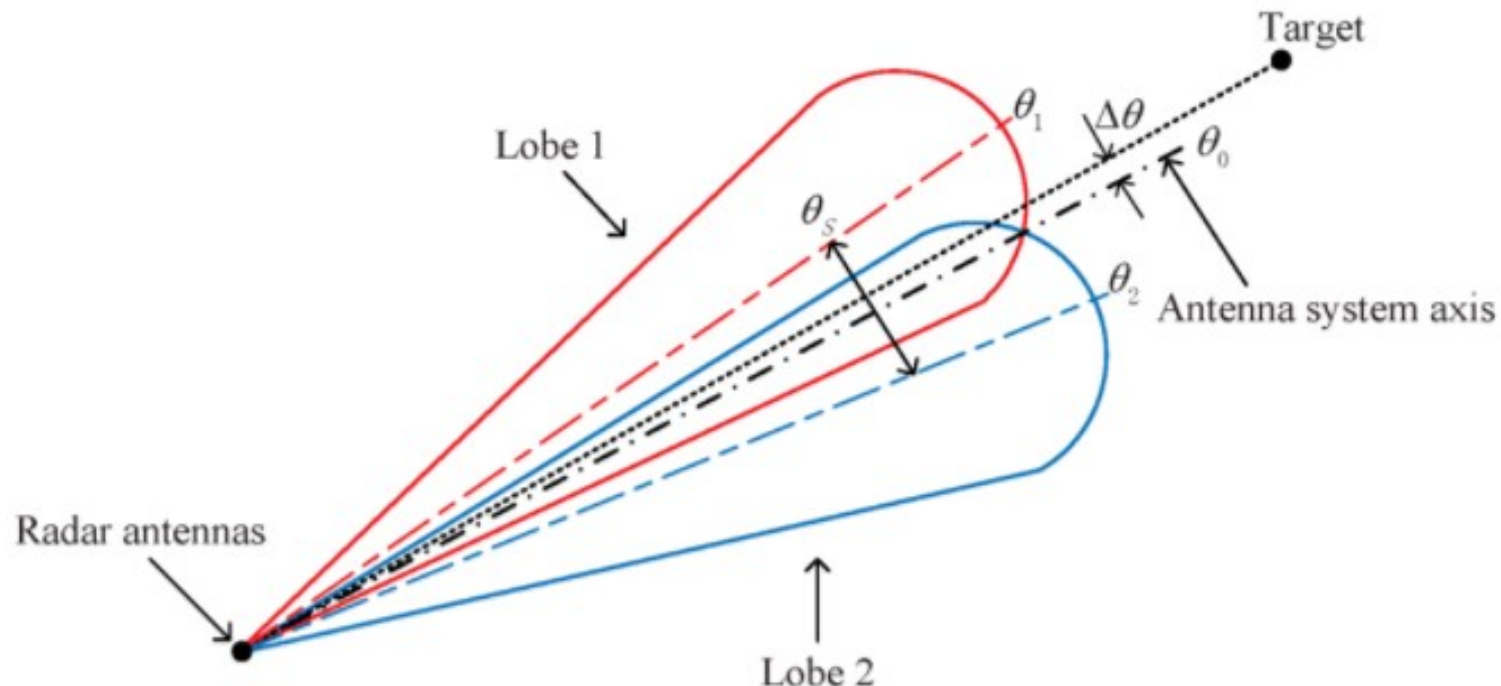

Figure 3: Amplitude comparison monopulse example [9]

Here, the angle $\theta_{\mathrm{s}}$ is the squint angle, which is 45 degrees in the case of this thesis. The tracking axis of the antenna system is along the dotted line from the radar antennas to the target as shown in Figure 3: Amplitude comparison monopulse example [9].

The math behind finding the ratio of the difference signal to the summation signal is shown below:

$$
\begin{aligned}
& d=\frac{v_{1}-v_{2}}{\sqrt{2}} \\
& s=\frac{v_{1}+v_{2}}{\sqrt{2}}
\end{aligned}
$$

Where $\mathrm{d}$ is the difference signal, $\mathrm{s}$ is the summation signal, and $\mathrm{v} 1$ and $\mathrm{v} 2$ are the signals received by two antennas connected to two different channels. If $v_{1} \geq v_{2}$, both channels have the same phase. Alternatively, if $v_{1}<v_{2}$, the channels have a $180^{\circ}$ phase difference. Here, the term phase does not necessarily refer to the phase domain. Instead, it refers to whether or not a target is on the antenna's tracking axis. If both channels have 
the same phase, the target is on the tracking axis and if the phase is 180 degrees the target is not on the tracking axis.

The $\sqrt{2}$ term in the equations above comes from assuming a hybrid junction circuit with passive lossless devices that form the sum and difference signals in real time. However, since the DF is done using the ratio of these two signals, the $\sqrt{2}$ term will come out of the ratio as shown below [6]:

$$
\frac{d}{s}=\frac{\frac{v_{1}-v_{2}}{\sqrt{2}}}{\frac{v_{1}+v_{2}}{\sqrt{2}}}=\frac{v_{1}-v_{2}}{v_{1}+v_{2}}=\frac{1-\frac{v_{2}}{v_{1}}}{1+\frac{v_{2}}{v_{1}}}
$$

The ratio of the difference and summation signals is proportional to the target's angle off the boresight axis. Therefore, this ratio can then be used to determine the resultant angular displacement between the antennas boresight and the line of sight to the source of the received signal. The ratio is known as the error signal. This signal is used by closed loop monopulse systems to move a servo in the direction of a target. In open loop monopulse systems this ratio provides an indicator of where the target is, but it will not change the positioning of the antennas boresight [6]. In the case of this thesis, since amplitude comparison monopulse is done in post-processing, it does not make sense to use closed-loop monopulse since the error signal is not calculated in real time and can't move a servo in the direction of a target in real time. Therefore, open-loop amplitude comparison monopulse is used as the DF method for this thesis.

Figure 4: Zoomed in error signal (Error signal voltage vs Azimuth angle in ${ }^{\circ}$ ) below shows the ratio of the difference signal to the sum signal. 


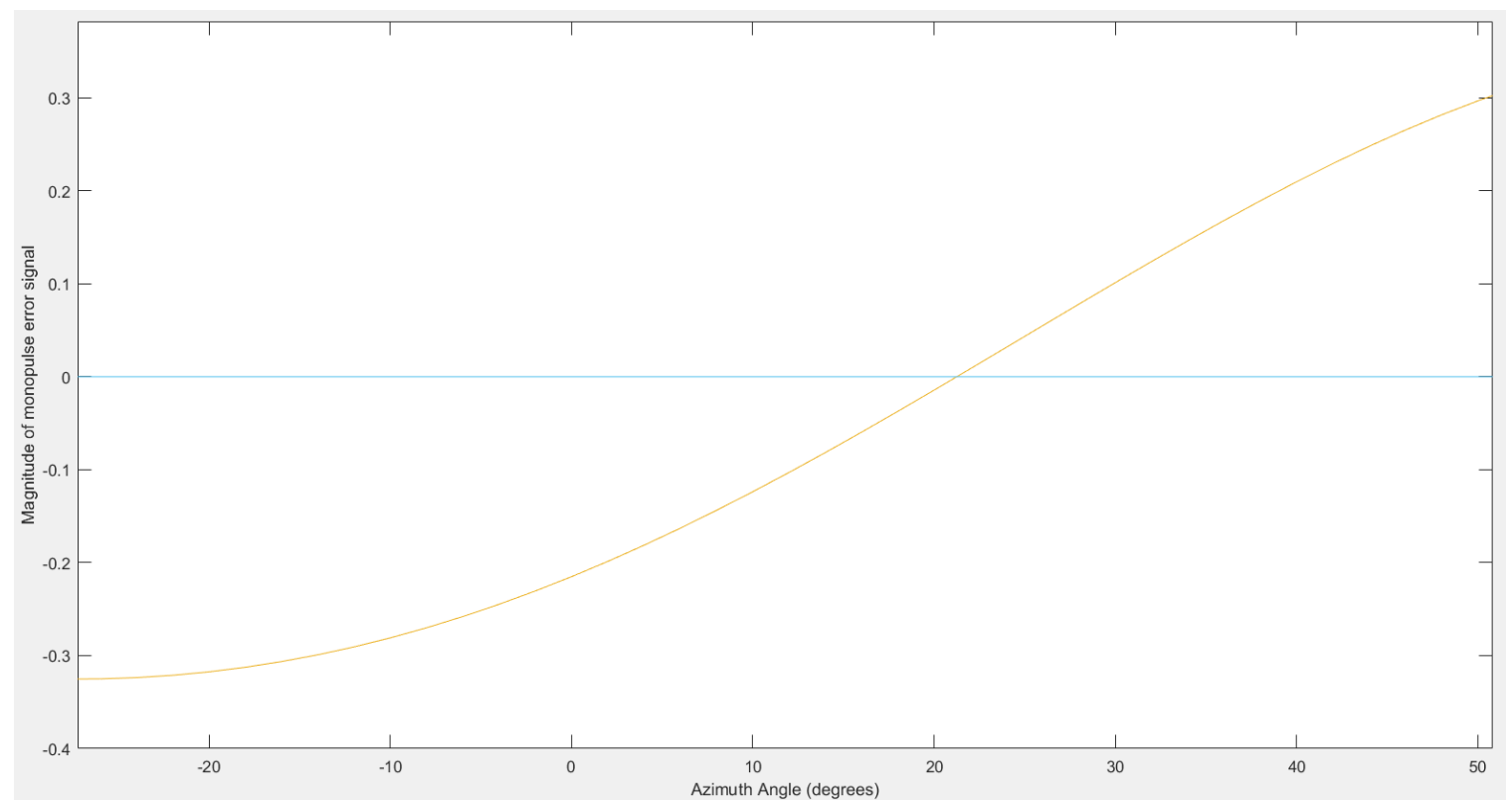

Figure 4: Zoomed in error signal (Error signal voltage vs Azimuth angle in ${ }^{\circ}$ )

The point where the error signal reaches 0 is the angle where the antenna tracking axis is located in both closed and open-loop monopulse. In a closed-loop system, the receiving antennas are moved until the target is located somewhere on the antenna tracking axis. In an open-loop system, the receiving antennas are stationary. The error using this method can be determined experimentally by setting up a transmitting antenna in an anechoic chamber at a known angle or by using the known ratio of the receiving antennas radiation pattern at each angle in the plane of interest.

The error associated with amplitude comparison monopulse can come from the antenna being used (beamwidth or phase center variation with polarization and/or frequency), errors in the manufacturing process when building the antenna, and losses in cables and ports. There are also some system based errors such as thermal noise and SNR which can affect the accuracy of amplitude comparison monopulse. 
The accuracy of the error signal can be qualitatively defined as more accurate when the error gradient is larger [10]. The error signal's gradient is a measure of the ability of a monopulse system to track incremental changes in the error signal such that it can accurately specify the target's bearing angle.

Amplitude comparison monopulse can be affected by the ambient temperature of its systems surrounding environment. This is important to note when introducing amplitude comparison monopulse to a UAS. Since UAS's fly up to altitudes around 10000 feet high, they rise through the troposphere. As shown in the Figure 5: Altitude $(\mathrm{km})$ vs Temperature $\left({ }^{\circ} \mathrm{K}\right)[11]$, this leads to a large reduction in ambient temperature from a system that would operate on the ground, or at a 10000 feet altitude. 


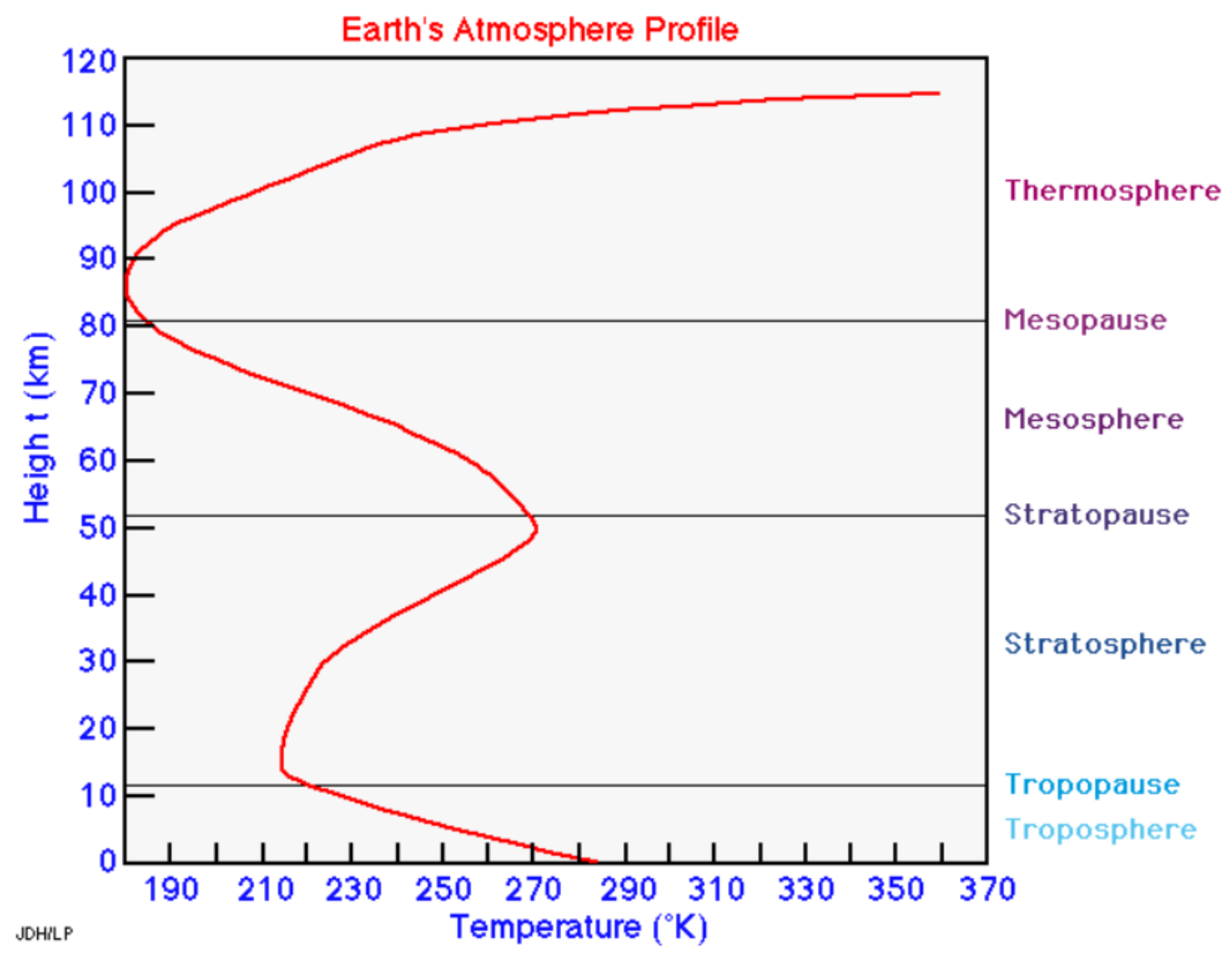

Figure 5: Altitude ( $\mathrm{km})$ vs Temperature ( $\left.{ }^{\circ} \mathrm{K}\right)$ [11]

There is approximately a $30{ }^{\circ} \mathrm{K}$ difference between ground level and a $10000 \mathrm{feet} / 3 \mathrm{~km}$ altitude. This means that an amplitude comparison monopulse UAS flying at an altitude around $3 \mathrm{~km}$ above the Earth's surface would be operating in an ambient temperature approximately $30{ }^{\circ} \mathrm{K}$ cooler than a tracking radar amplitude comparison monopulse system.

This reduces the thermal noise power and therefore the total noise of the receiver described by kTB, where $\mathrm{k}$ is Boltzmann's constant $\left(1.38 \times 10^{-23} \mathrm{~J} /{ }^{\circ}\right), \mathrm{T}$ is the temperature in ${ }^{\circ} \mathrm{K}$, and $\mathrm{B}$ is the bandwidth [5]. With a receiver that has an instantaneous bandwidth of $20 \mathrm{MHz}$ for example, the difference in noise power is shown below: 


$$
\begin{gathered}
10 * \log \left(1.38 * 10^{-23} * 20 * 10^{6} * 280\right)-10 * \log \left(1.38 * 10^{-23} * 20 * 10^{6} * 250\right) \\
\cong 0.4922 d B
\end{gathered}
$$

Therefore, in a UAS flying at an altitude of about 10000 feet, the thermal noise of a receiver is reduced by approximately half a $\mathrm{dB}$.

\subsubsection{Comparing Amplitude Comparison Monopulse to Other Monopulse Methods}

To get an idea of what the theoretical error of a rhombic amplitude comparison monopulse system would be, a table is given below outlining several different monopulse systems tracking errors.

\begin{tabular}{|c|c|c|c|c|c|c|c|c|c|c|c|c|c|c|c|}
\hline \multirow{3}{*}{$\begin{array}{c}\text { System Type } \\
\text { Average Error } \\
\text { Gradient Over a } \\
\pm 30^{\circ} \text { Field of View }\end{array}$} & \multirow{2}{*}{\multicolumn{3}{|c|}{$\begin{array}{l}\text { 2-Channel Amplitude } \\
\text { and Phase Comparison }\end{array}$}} & \multirow{2}{*}{\multicolumn{3}{|c|}{$\begin{array}{l}\text { 3-Channel Amplitude } \\
\text { and Phase Comparison }\end{array}$}} & \multirow{2}{*}{\multicolumn{3}{|c|}{$\begin{array}{l}\text { Amplitude } \\
\text { Comparison }\end{array}$}} & \multicolumn{6}{|c|}{ Phase Comparison } \\
\hline & & & & & & & & & & \multicolumn{3}{|c|}{$\begin{array}{l}\text { 0.85-Wavelength } \\
\text { Baseline }\end{array}$} & \multicolumn{3}{|c|}{$\begin{array}{l}\text { 3-Wavelength } \\
\text { Baseline }\end{array}$} \\
\hline & \multicolumn{3}{|c|}{$0.79 \mathrm{~dB} / \mathrm{deg}$} & \multicolumn{3}{|c|}{$\begin{array}{c}0.85 \mathrm{~dB} / \mathrm{deg} \\
(0.8 \text {-wavelength } \\
\text { element spacing) }\end{array}$} & \multicolumn{3}{|c|}{$\begin{array}{c}0.44 \mathrm{~dB} / \mathrm{deg} \\
\left(70^{\circ} 3-\mathrm{dB}\right. \\
\mathrm{BW} \text { and } 45^{\circ} \\
\text { element offset })\end{array}$} & \multicolumn{3}{|c|}{$5.1 \mathrm{deg} / \mathrm{deg}$} & \multicolumn{3}{|c|}{$18 \mathrm{deg} / \mathrm{deg}$} \\
\hline Error Parameter & $\mathrm{dB}$ & $\begin{array}{c}\text { spatial } \\
\text { deg }\end{array}$ & $(\mathrm{deg})^{2}$ & $\mathrm{~dB}$ & $\begin{array}{l}\text { spatial } \\
\text { deg }\end{array}$ & $(\mathrm{deg})^{2}$ & $\mathrm{~dB}$ & $\begin{array}{c}\text { spatial } \\
\text { deg }\end{array}$ & $(\mathrm{deg})^{2}$ & $\begin{array}{c}\text { electrical } \\
\text { deg }\end{array}$ & $\begin{array}{c}\text { spatial } \\
\text { deg }\end{array}$ & $(\mathrm{deg})^{2}$ & $\begin{array}{l}\text { electrical } \\
\text { deg }\end{array}$ & $\begin{array}{c}\text { spatial } \\
\text { deg }\end{array}$ & $(\mathrm{deg})^{2}$ \\
\hline $\begin{array}{l}\text { Polarization and Antenna } \\
\text { Pattern Errors }\end{array}$ & - & 2.45 & 6.0 & 1.3 & 1.53 & 2.35 & 2.2 & 5.00 & 25.0 & 4.0 & 0.79 & 0.62 & 1.3 & 0.83 & 0.07 \\
\hline $\begin{array}{l}\text { RF Beam-Forming } \\
\text { Network Errors }\end{array}$ & 0.5 & 1.39 & 1.92 & 0.5 & 0.59 & 0.35 & - & - & - & - & - & - & - & - & - \\
\hline $\begin{array}{l}\text { Thermal Noise Error } \\
\text { (Signal-to-Noise Ratio } \\
\text { of } 30 \mathrm{~dB} \text { and } 1 \text { Pulse) }\end{array}$ & - & 1.17 & 1.36 & - & 0.75 & 0.56 & - & 2.73 & 7.47 & 0.05 & 0.01 & 0.0001 & 0.05 & 0.0028 & - \\
\hline $\begin{array}{l}\text { Calibrated Receiver } \\
\text { Imbalance Error } \\
\text { (Includes } 0.25 \mathrm{~dB} \\
\text { Quantization Error) }\end{array}$ & 1.0 & 2.76 & 7.63 & 1.0 & 1.18 & 1.39 & 1.0 & 2.27 & 5.15 & 10.0 & 2.5 & 6.25 & 10.0 & 0.55 & 0.31 \\
\hline $\begin{array}{l}\text { Mechanical Alignment } \\
\text { Errors }\end{array}$ & - & 0.1 & 0.01 & & 0.1 & 0.01 & - & 0.1 & 0.01 & - & 0.1 & 0.01 & - & 0.1 & 0.01 \\
\hline Sum of the Variances & & & 16.92 & & & 4.66 & - & - & 37.63 & - & - & 6.88 & - & - & 0.39 \\
\hline Rms (1 $\sigma)$ Bearing & & $4.12^{\circ}$ & & & $2.16^{\circ}$ & & & $6.12^{\circ}$ & & & $2.62^{\circ}$ & & & $0.63^{\circ}$ & \\
\hline
\end{tabular}

Table 1: Amplitude comparison monopulse bearing error by system type [10] 
Looking at the table above, the closest situations to this thesis are the 2-channel amplitude and phase comparison case and the amplitude comparison case. It is important to note that the angles given in the bottommost row of the table are standard deviations. Therefore, it is fair to assume that most of the time the bearing error of the methods in the table above will be on the order of the given angle, or less. All of the values in the table above are based on systems that use one pulse, or one data point to find the bearing of a target. In this thesis, the same method is used. However, if this thesis topic becomes a product, an average of multiple pulses/data points would be used, further improving the bearing accuracy.

The amplitude comparison case in the table above has a 45 degree elemental offset which is the same as this thesis. However, in the following sections, a variable HPBW over a large frequency range will be presented. With a lower HPBW than the 70 degrees in the table above, the received signal strength should be stronger in the main lobe at the $-3 \mathrm{~dB}$ point (assuming a rhombic were used for both HPBWs). If this is the case, using rhombics should provide a slight improvement on the 6.12 degree error given in the table above.

A visual representation of the effect of the SNR and target angle-off-boresight is shown below for an amplitude comparison monopulse system: 


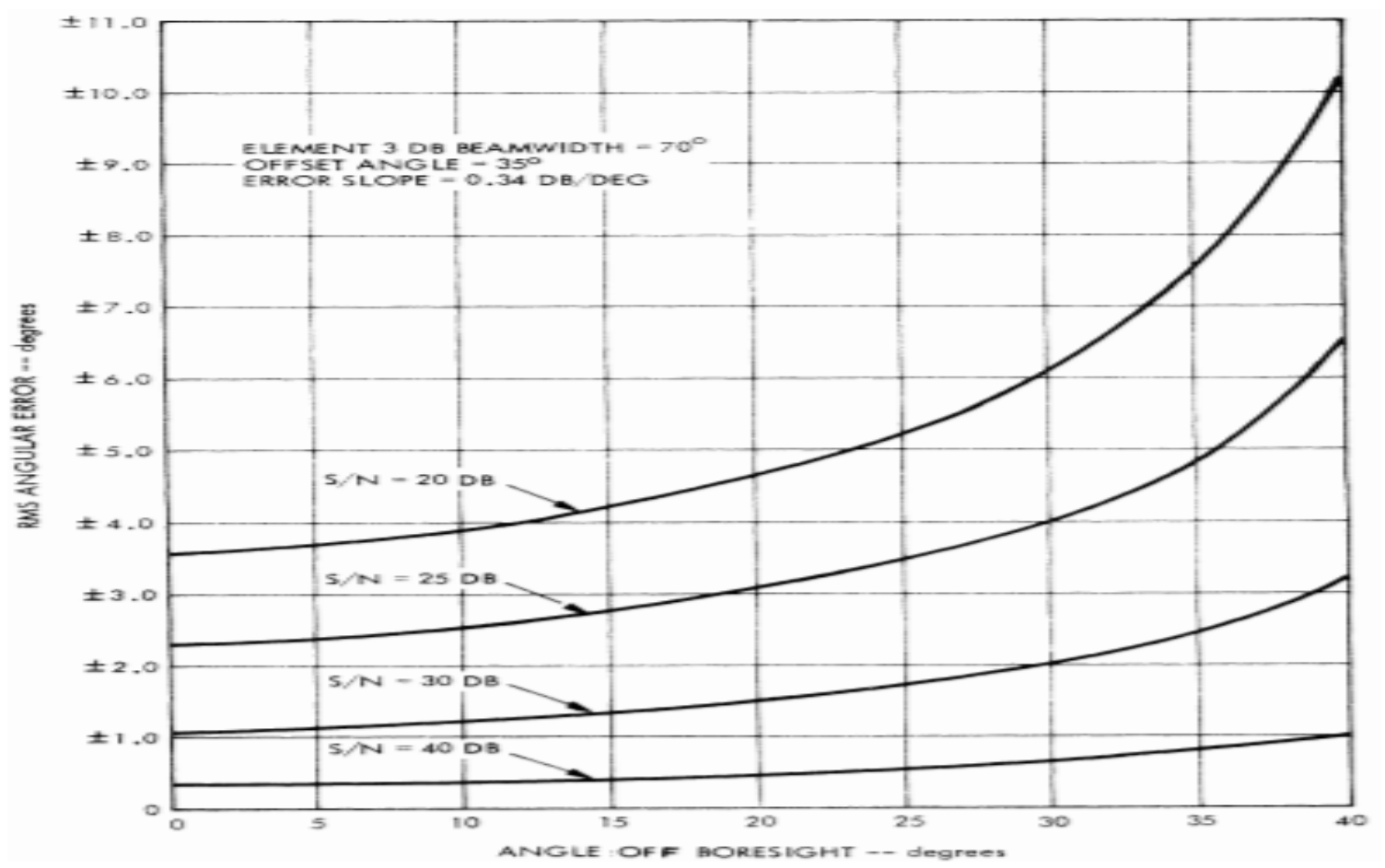

Figure 6: RMS angular error vs target angle-off-boresight for changing SNR [10]

This shows a heavy dependency of amplitude comparison monopulse's bearing accuracy on SNR. This behaviour makes sense since noise can have a large impact on the amplitude measurements from an antenna if the SNR is low enough. The same could also be said about phase noise in phase comparison monopulse.

While the Figure 6: RMS angular error vs target angle-off-boresight for changing SNR [10] is a good illustration of how important SNR is to the accuracy of amplitude comparison monopulse it is also important to note that for this UAS application, there will be less noise thus improving the SNR and bearing accuracy. There is less noise because the SNR curves in Figure 6: RMS angular error vs target angle-off-boresight for changing SNR [10] are under the assumption that there is a set of circuit components which are doing the error signal calculation. This introduces several additional sources of noise that do not exist in this thesis' implementation since the error signal calculations are 
done in post-processing. A block diagram is shown below which contains these circuit components for a 4 channel system.

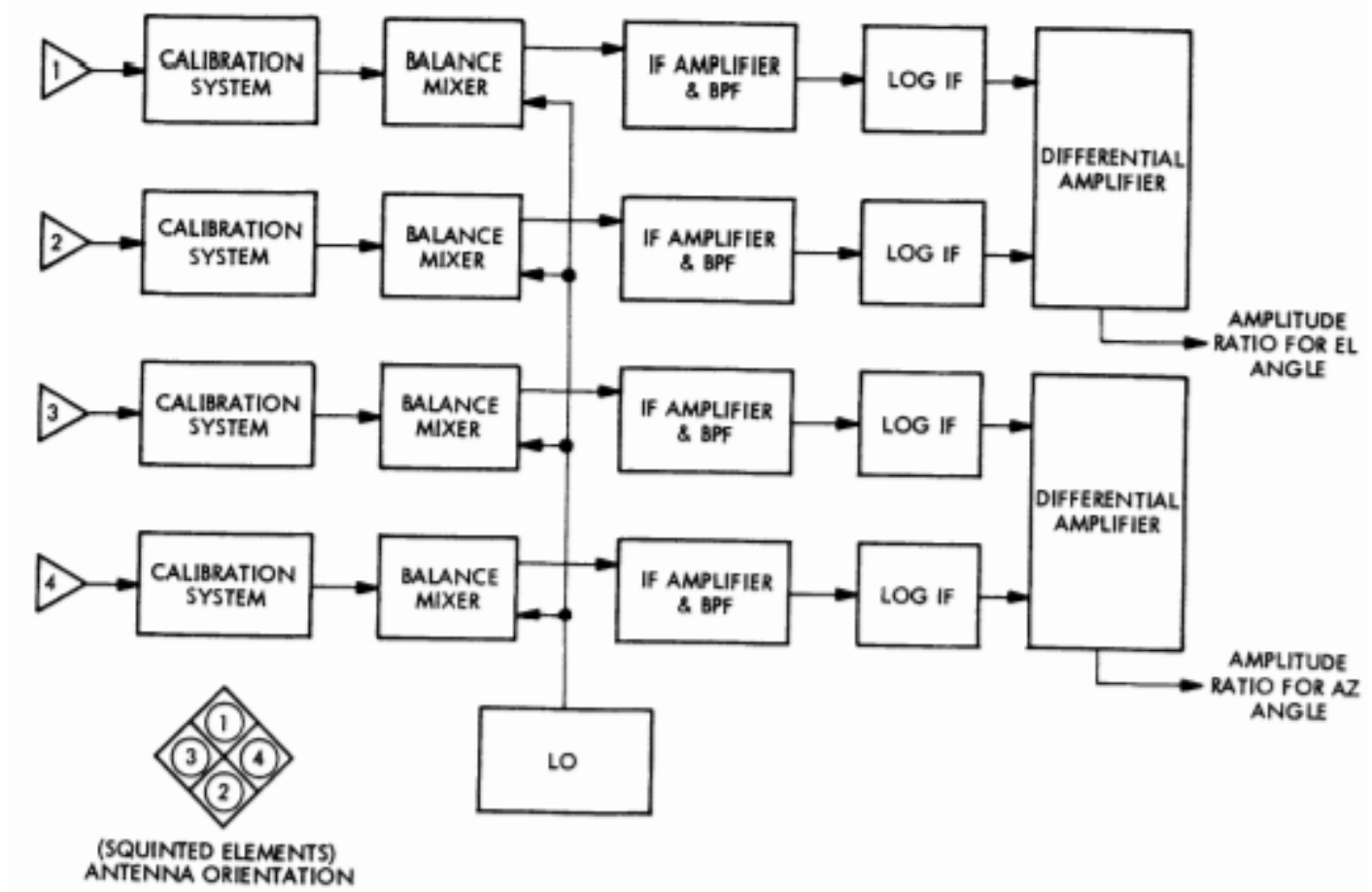

Figure 7: Block diagram of a monopulse hardware based system [10]

The circuit components shown in Figure 7: Block diagram of a monopulse hardware based system [10] are not needed in this thesis' amplitude comparison monopulse configuration since it is an open-loop configuration. Another required circuit in the closed-loop configuration is called an AGC (Automatic Gain Control) circuit. The AGC circuit is responsible for ensuring that the closed-loop servo system is not affected by a changing received signal amplitude [5]. Figure 8: Open loop vs closed loop monopulse system block diagrams shows a block diagram comparison between this thesis' open-loop monopulse configuration and a close-loop configuration in terms of system components. 
Open-loop monopulse:

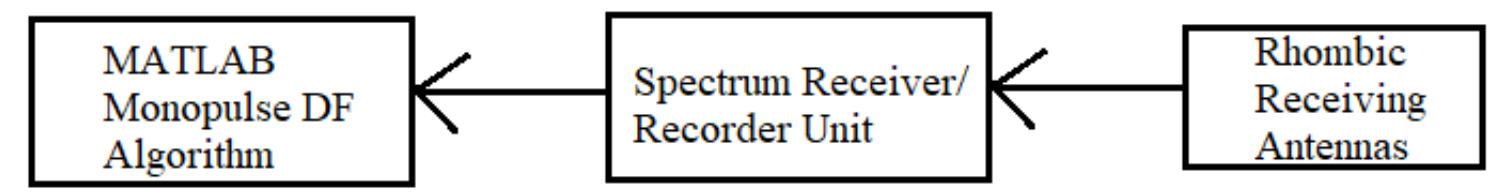

Closed-loop monopulse:

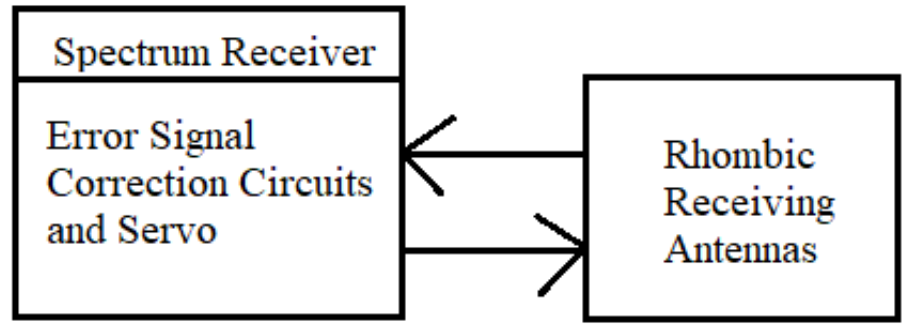

Figure 8: Open loop vs closed loop monopulse system block diagrams

The circuit components are not needed in an open-loop configuration because the summation, difference, and error signals can be produced in post-processing with a tool such as MATLAB instead of the error signal correction circuits. This not only reduces the amount of circuitry required on board a UAS (reducing size and weight), but also reduces the noise which can improve the performance of amplitude comparison monopulse.

Noise, multipath, and path loss in this UAS is improved when compared to a tracking radar monopulse system. Therefore, it is fair to say that in a 2 channels system, there should not be a large discrepancy in bearing accuracy between amplitude comparison monopulse and phase comparison monopulse. This makes amplitude comparison monopulse an attractive option for DF of a 2 channel UAS.

It is important to note that there is no strict minimum signal or noise floor for an amplitude comparison monopulse application. This is because the bearing measurement is done based on a ratio of the difference to summation signals. If a received signal is low in amplitude for both channels, the method would still work just as well as it would if 
those signals were high in amplitude assuming the signal stays above the systems noise floor. However, the systems noise floor does not necessarily need to have a minimum. 


\section{3 - Rhombic Antenna Design}

\subsection{Introduction}

The design of the rhombic antennas must fit in a $32.4 \mathrm{~cm}$ x $19.4 \mathrm{~cm}$ area and make use of amplitude comparison monopulse as a means of DF. In order to accomplish this, design practices and characteristics of rhombic antennas must be understood. Once these practices are understood, the size constraints of the UAS and the DF requirements of the thesis can be considered.

Rhombics are wire antennas in the shape of a rhombus with a terminating resistance connected to one end and a connection to a transmitter or receiver at the other. They can be fed from either side if there is a need, as they can be made bidirectional [12]. However, in the case of this thesis, only one direction at a time will be needed, therefore the rhombic is terminated. Typically rhombics are used at lower frequencies (such as the HF band) and are tens of feet long, wide, and tall [3].

Another important benefit of rhombic antennas as it relates to DF is that they do not require constant calibration as most DF antennas do. DF antennas typically require calibration based on temperature change either on an aircraft and/or the air below the aircraft. Since rhombic antennas are wire antennas, their performance is not greatly affected by changes in temperature. Therefore, rhombic antennas do not need to be regularly calibrated. A disadvantage of rhombics is that there is a considerably large secondary or back lobe even under good design conditions [3]. Another disadvantage is that there is a loss of power in the terminating load of a rhombic [3]. This makes the received power a bit lower, but the relatively high gain of rhombics helps offset this issue. 
Rhombic antennas are made up of two $\mathrm{V}$ antennas. $\mathrm{V}$ antennas have radiation patterns which are controlled by the length of each side. When two $\mathrm{V}$ antennas are connected together (usually with a terminating resistor), a rhombic antenna is formed. Rhombic antennas are more desirable than $\mathrm{V}$ antennas for nonresonant and unidirectional radiation patterns [13]. Since amplitude comparison monopulse works better with a higher amplitude resolution (more power concentrated in the main lobe), rhombics are a better choice when compared to $\mathrm{V}$ antennas since rhombics typically have a more directional radiation pattern.

The rhombic antennas need to be designed in such a way that the 45 degree squint requirement for the amplitude comparison monopulse DF technique is met. The gain of the rhombic should also be as reasonable as it can be given the size and bandwidth constraints imposed by the thesis requirements.

Since such a large bandwidth is to be covered by the rhombics, the band was split into two bands. This includes an upper band from 1-6 GHz and a lower band from 0.6-1 GHz. The antennas had to be designed for a two channel receiver to achieve a solution for the amplitude comparison monopulse algorithm. This means that there needs to be four antennas. Two of the antennas are for the two channels in the upper band and two are for the two channels in the lower band. A software-defined method for switching between upper and lower frequency bands for the antennas is left as future work. This softwaredefined method of switching would be implemented by sending a digital signal from the UAS' receiver to a switch controlling which antennas are being used. Figure 9: Rhombic antenna orientation showing the rhombic antenna orientation is shown below: 


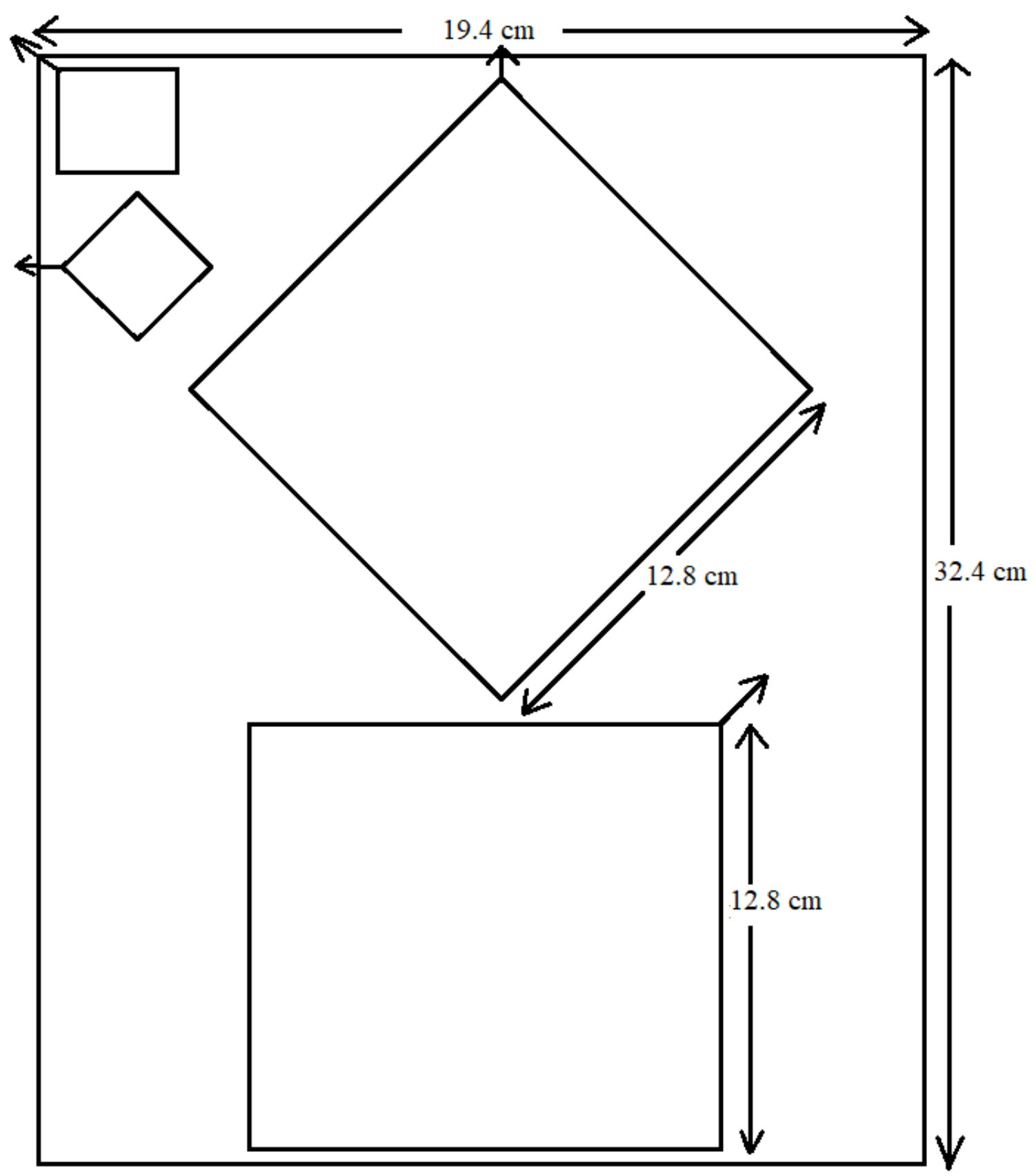

Figure 9: Rhombic antenna orientation

Here, the rhombic antennas are oriented such that there is a 45 degree squint angle between rhombics of the same size. For example, the bottommost rhombic has a main lobe pointing upwards and to the right 45 degrees while the other $12.8 \mathrm{~cm}$ leg length rhombic has its main lobe pointing directly upwards. This will give a squint angle of approximately 45 degrees, which allows the rhombic antennas to satisfy the squint angle 
requirement outlined in chapter 2. The $12.8 \mathrm{~cm}$ size shown in Figure 9: Rhombic antenna orientation is slightly less than the maximum size given the size constraints of this thesis. This is to allow room for any cables that are necessary to connect the components of the UAS together. Note that the smaller antennas can have leg lengths no larger than $3.5 \mathrm{~cm}$ for the same reason.

\subsection{Rhombic Design Details}

The main problem that had to be solved when designing the rhombic antenna was how to take the typical large HF band (3-30 MHz) rhombic design and fit it into a $32.4 \mathrm{~cm} \times 19.4$ $\mathrm{cm}$ area. To do this, the process of how to design the radiation pattern of a typical rhombic antenna and how to optimize its gain had to be understood. Since amplitude comparison monopulse is being used as the DF technique, the radiation pattern becomes more important than the gain assuming the gain is reasonable. Therefore, this section will focus largely on the design of the rhombic's radiation pattern.

Since a wide main lobe is wanted for amplitude comparison monopulse, the number of sidelobes in the radiation pattern should be minimized in order to improve the accuracy of a DF measurement. For typical rhombics, there are no sidelobes when the leg length L (or side length) of the rhombic are half a wavelength long. There are $\mathrm{n}$ additional sidelobes for each half wavelength increase in the leg length L of the rhombic [3]. Figure 10: Large scale rhombic antenna [3] shows a model of a large rhombic with labelled leg length L. 


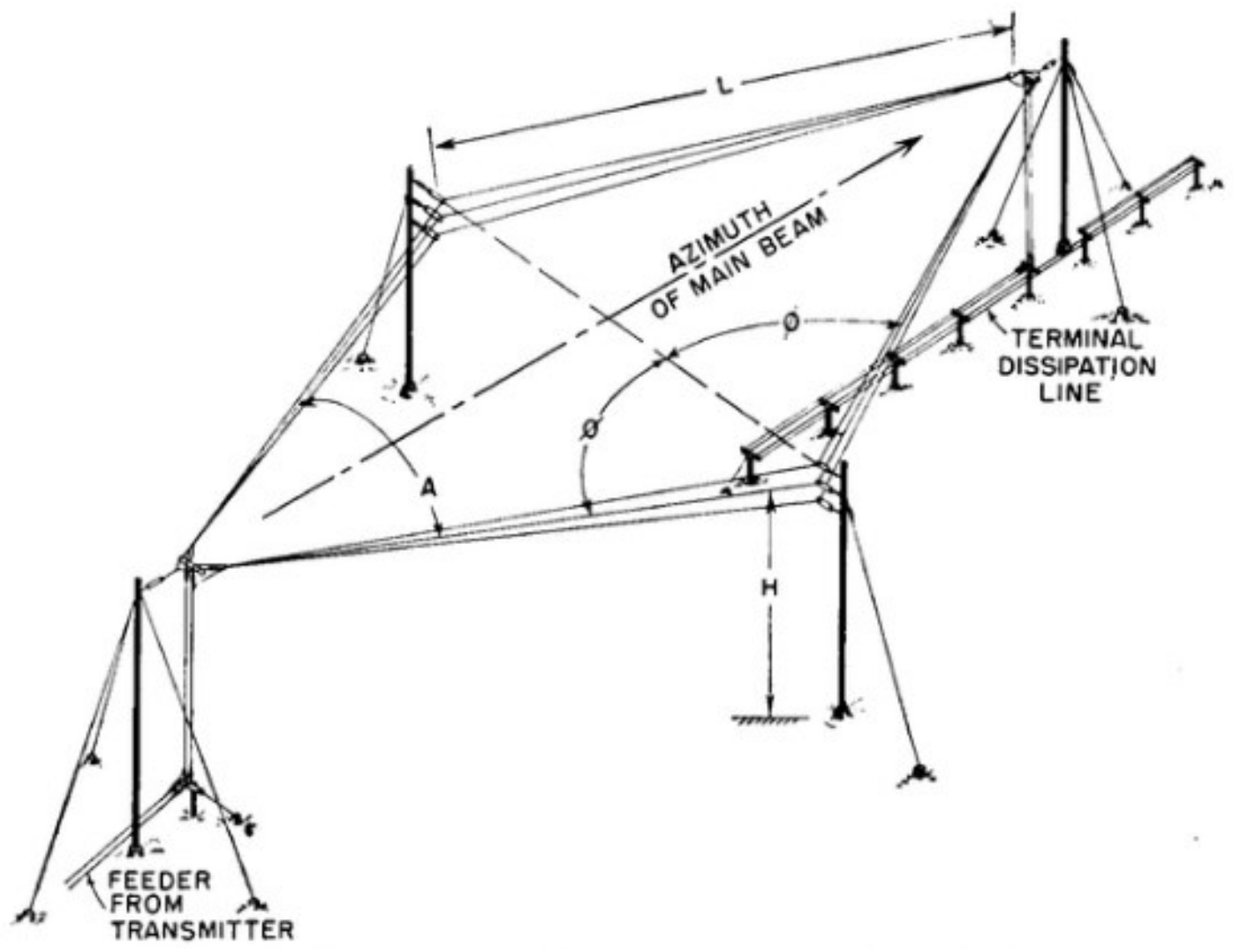

Figure 10: Large scale rhombic antenna [3]

The terminal dissipation line shown in Figure 10: Large scale rhombic antenna [3] is replaced in the case of this thesis by a terminating resistance. The terminal dissipation line typically had a non-inductive resistance of $600 \Omega$ [3]. This value is used as a starting terminating resistance for the rhombic simulations in chapter 4 . The feeder from the transmitter portion of Figure 10: Large scale rhombic antenna [3] in the UAS's case goes to an SMA (SubMiniature Version A) connector which is hooked up to the UAS's receiver. There will be more details regarding the terminating resistance in the testing section.

The angle between the legs of each rhombic was chosen to be 90 degrees. This angle is called the apex angle given by A in Figure 10: Large scale rhombic antenna [3]. As the 
apex angle is increased, the main lobe's HPBW increases [14]. It will be shown in chapter 4, that a 90 degree apex angle is larger than necessary to obtain a HPBW suitable for a 45 degree squint angle. The fine tuning of the apex angle is left as future work.

It should also be noted that there are three wires in Figure 10: Large scale rhombic antenna [3] along the perimeter of the rhombic. This is a more optimal way of designing the rhombics in general [3]. However it is not a practical design to use with a UAS since there can't be three symmetric wires as shown in Figure 10: Large scale rhombic antenna [3] without taking up far more space in the $\mathrm{z}$ direction and putting the rhombic antennas on an angle. There is only about $12 \mathrm{~cm}$ of space in the $\mathrm{z}$ direction to work with (the space available between the bottom of the UAV and the ground when grounded), so all of the space that can be saved in the $\mathrm{z}$ direction becomes relevant. It is relevant since a half wavelength separation should be between the rhombic and the bottom of the UAV. This separation is standard practice when using multiple antennas in a system since the closer antennas are together the more they couple. In order to find the minimum frequency at which there is a half wavelength separation while allowing a small separation of $2 \mathrm{~cm}$ between the ground and the bottom of the UAS, a half wavelength of $10 \mathrm{~cm}$, or $0.1 \mathrm{~m}$ can be used as follows:

$$
0.5 \lambda=0.1 \mathrm{~m} \rightarrow f=\frac{c}{\lambda}=\frac{3 \times 10^{8} \mathrm{~m} / \mathrm{s}}{0.2 \mathrm{~m}}=1.5 \mathrm{GHz}
$$

Therefore, with a $2 \mathrm{~cm}$ gap between the ground and the UAS a half wavelength separation between the rhombic antennas and the UAS's ground plane can be achieved at $1.5 \mathrm{GHz}$ or higher. 
It is important to note that this design is based on a single wire rhombic design. In many rhombic designs "curtain" rhombics are used. These are rhombics which have two or three wires per leg of the rhombic [15]. Curtain rhombics have a smaller termination loss making them more efficient for transmitting and receiving. However, for this thesis, the single wire-type was used in order to save space on the UAV. 


\section{4 - Simulations}

\subsection{Introduction}

There are two major points of interest that need to be simulated for the UAS; the rhombic antenna design and the approximate expected DF accuracy. The rhombic antenna was simulated using HFSS (High Frequency Structure Simulator) which allows users to design and simulate pre-built or custom antennas. DF simulations were performed in MATLAB which allowed for importing radiation pattern data from HFSS in CSV (Comma-Separated Values) format and creating custom DF scenarios.

For the HFSS simulations, the radiation pattern characteristics were simulated first. This was done to ensure that the rhombic antenna will have a large portion of the received power in the main lobe for the amplitude comparison monopulse DF. Having no sidelobes in the rhombics radiation pattern allows for this to occur. Therefore, the task was split into two parts; finding a way to eliminate sidelobes and simulating the radiation pattern with a known number of sidelobes. Then, the gain and S11 were optimized for the upper and lower frequency bands.

The DF simulations are performed to give the reader a representation of what parameters affect the target bearing error when using amplitude comparison monopulse. These simulations utilize data from the HFSS simulations. Error sources that could be incorporated into the MATLAB DF simulation include the turning error of the aircraft $(+/-0.1$ degree per turn of the UAV, it is assumed that this error averages to 0$)$, the free space path loss, and noise. This was modeled in MATLAB using its phased array toolbox and the antenna toolbox. 


\subsection{HFSS Simulation Setup}

Figure 11: HFSS simulation layout of $12.8 \mathrm{~cm}$ leg length rhombic below shows the HFSS layout of the rhombic antenna that was simulated.

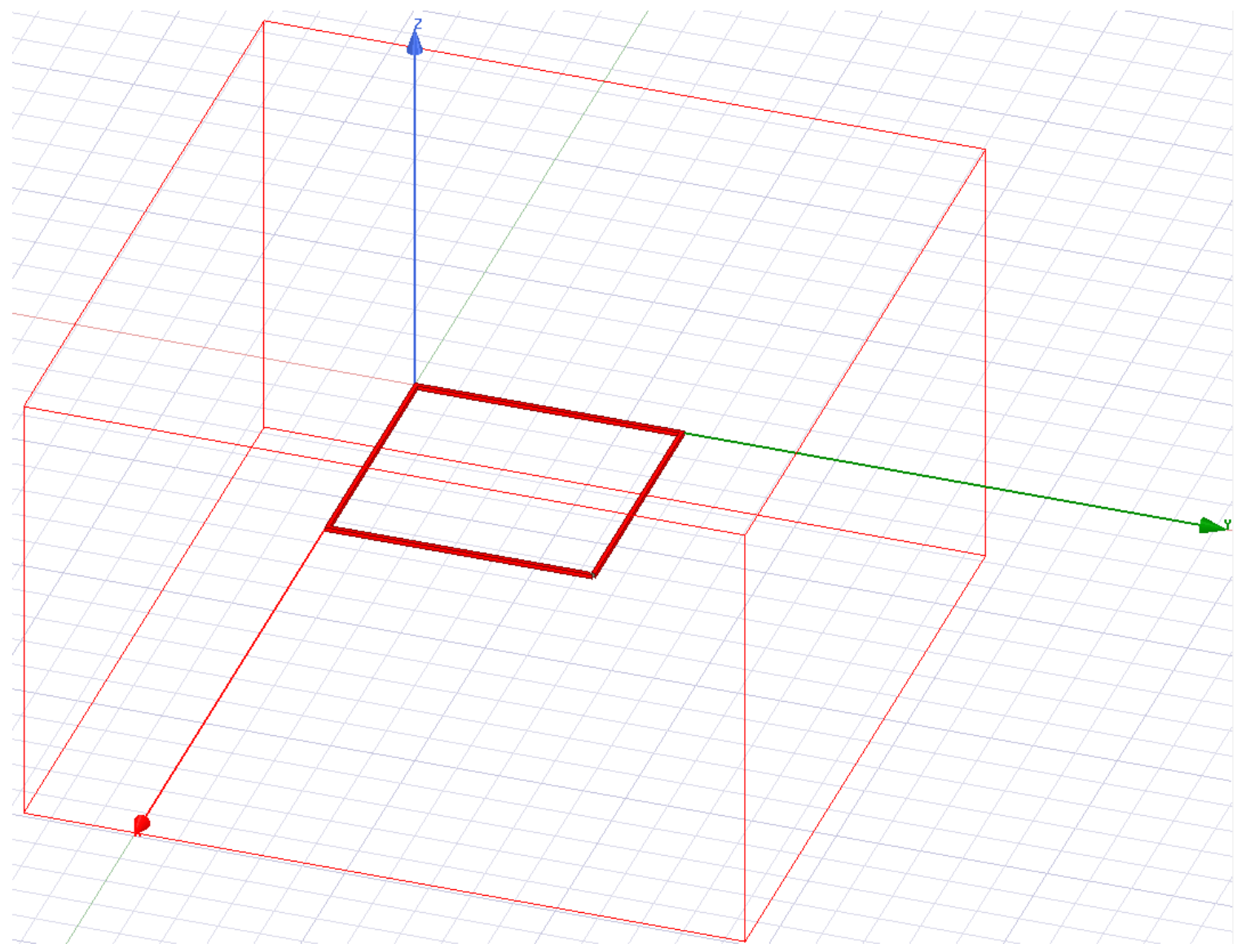

Figure 11: HFSS simulation layout of $12.8 \mathrm{~cm}$ leg length rhombic

As seen in Figure 11: HFSS simulation layout of $12.8 \mathrm{~cm}$ leg length rhombic, the rhombic simulations were performed with a rhombic antenna sitting on the azimuth axis. The rhombic antenna was configured to receive a signal at 45 degrees in the azimuth direction and 90 degrees in elevation.

The rhombus in Figure 11: HFSS simulation layout of $12.8 \mathrm{~cm}$ leg length rhombic represents the wires/legs of the rhombic antenna. The resistance of that port was initially set to $600 \Omega$ for the radiation pattern simulations since values between 600 and $800 \Omega$ were historically used as the terminating resistance for rhombics [3]. 
The red lines surrounding the antenna are from a PML (Perfectly Matched Layer) boundary. A PML is used to simulate a transmitted signal at a frequency specified by an HFSS user. Once a PML is added to a simulation, it can provide results for the radiation pattern and gain of the antenna.

Gain simulations were run based on a frequency sweep from $0.6-6 \mathrm{GHz}$ in steps of 10 MHz. Radiation pattern simulations were done at varying frequencies in the $0.6-6 \mathrm{GHz}$ range. The thickness of the wires of the antennas was set to $2 \mathrm{~mm}$ and the separation between the wires at the port was set to $1 \mathrm{~mm}$.

The elevation angle $\theta$ is set to 90 degrees for each simulation since the antenna will be flat at the bottom of the UAV. The radiation pattern simulations were two dimensional with 360 degrees of azimuth coverage at several different frequencies in the $0.6-6 \mathrm{GHz}$ range.

\subsection{HFSS Simulation Results}

\subsubsection{Number of Sidelobe Simulations}

First, simulations were run to confirm whether or not a standard method for determining the number of sidelobes of a rhombic antenna is valid in the frequency range of interest. This was important to check since rhombics have not previously been used in the upper portion of the $0.6-6 \mathrm{GHz}$ spectrum. This method finds the number of sidelobes by comparing half of the wavelength of a particular frequency of interest to the leg length of the rhombic. If the rhombics leg length is less than or equal to half a wavelength, there are no sidelobes. However, at integer multiples of half a wavelength the number of sidelobes increases by 1 [3]. For example, if a rhombics leg length is $0.7 \lambda$, the rhombic will have 1 sidelobe at that frequency. This means that when the rhombic leg lengths are 
scaled down by orders of magnitude (tens of meters to several centimeters) their sidelobe and main lobe power levels become less customizable, or desirable in some cases. The first simulation shown below shows a case at a frequency in which rhombics would typically operate $(200 \mathrm{MHz})$ with a wavelength of $0.3 \mathrm{~m}$. Note that all of the radiation pattern plots in this and the following section show the azimuth angle on the outer circle and the received power in $\mathrm{dBs}$ in the inner rings.
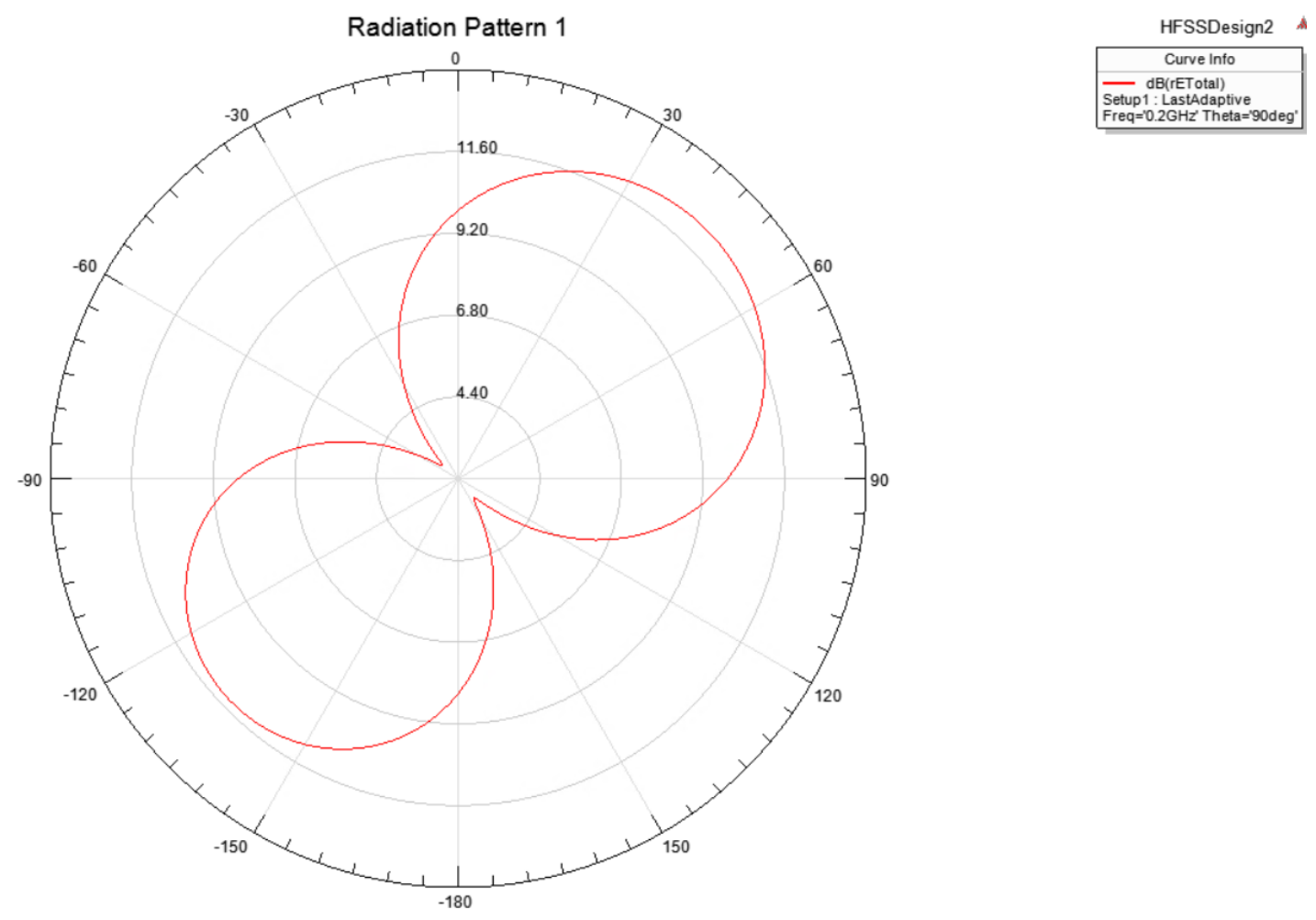

Figure 12: $200 \mathrm{MHz}, 0.3 \mathrm{~m}$ legs

$$
\text { In free space at } 200 \mathrm{MHz} \text {, the wavelength } \lambda=\frac{c}{f}=\frac{3 \times 10^{8} \mathrm{~m} / \mathrm{s}}{200 \times 10^{6} \mathrm{~Hz}}=1.5 \mathrm{~m}
$$

Since the simulation was run with a $0.3 \mathrm{~m}$ leg length, 0 sidelobes would be expected since half of the wavelength is $0.75 \mathrm{~m}$ at $200 \mathrm{MHz}$. This is the case here, so when the leg length is much smaller than half a wavelength the method of finding the number of 
sidelobes appears to be true. Other test cases shown below were run to check that this method works for cases with larger leg lengths relative to the receiving frequency.
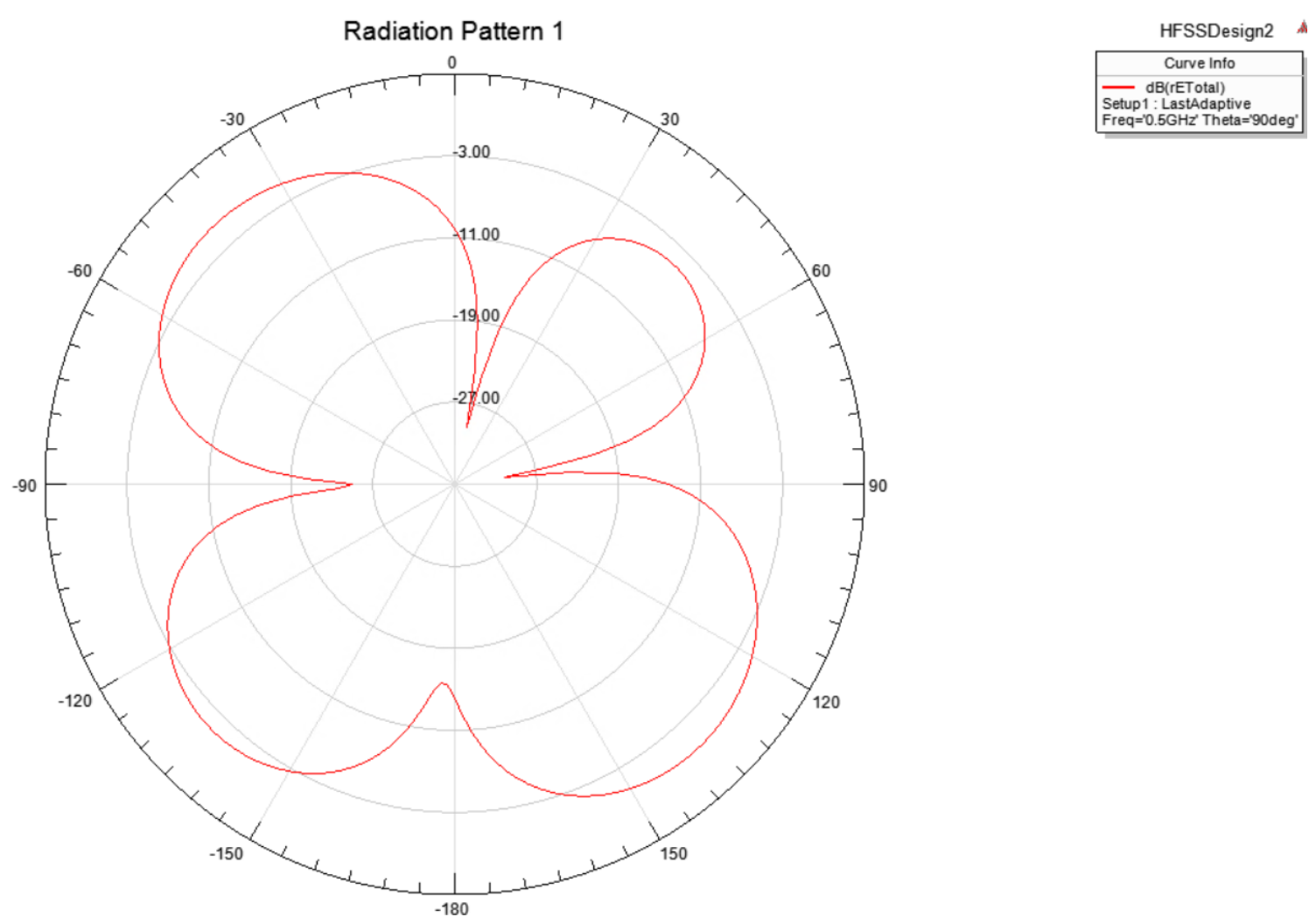

Figure 13: $500 \mathrm{MHz}, 0.3 \mathrm{~m}$ legs

$$
\text { In free space at } 500 \mathrm{MHz} \text {, the wavelength } \lambda=\frac{c}{f}=\frac{3 \times 10^{8} \mathrm{~m} / \mathrm{s}}{500 \times 10^{6} \mathrm{~Hz}}=0.6 \mathrm{~m}
$$

In Figure 13: $500 \mathrm{MHz}, 0.3 \mathrm{~m}$ legs, there is 1 sidelobe with a leg length which is exactly one half of the wavelength at $500 \mathrm{MHz}$. This makes sense since one would expect to see one sidelobe with half wavelength long legs. 

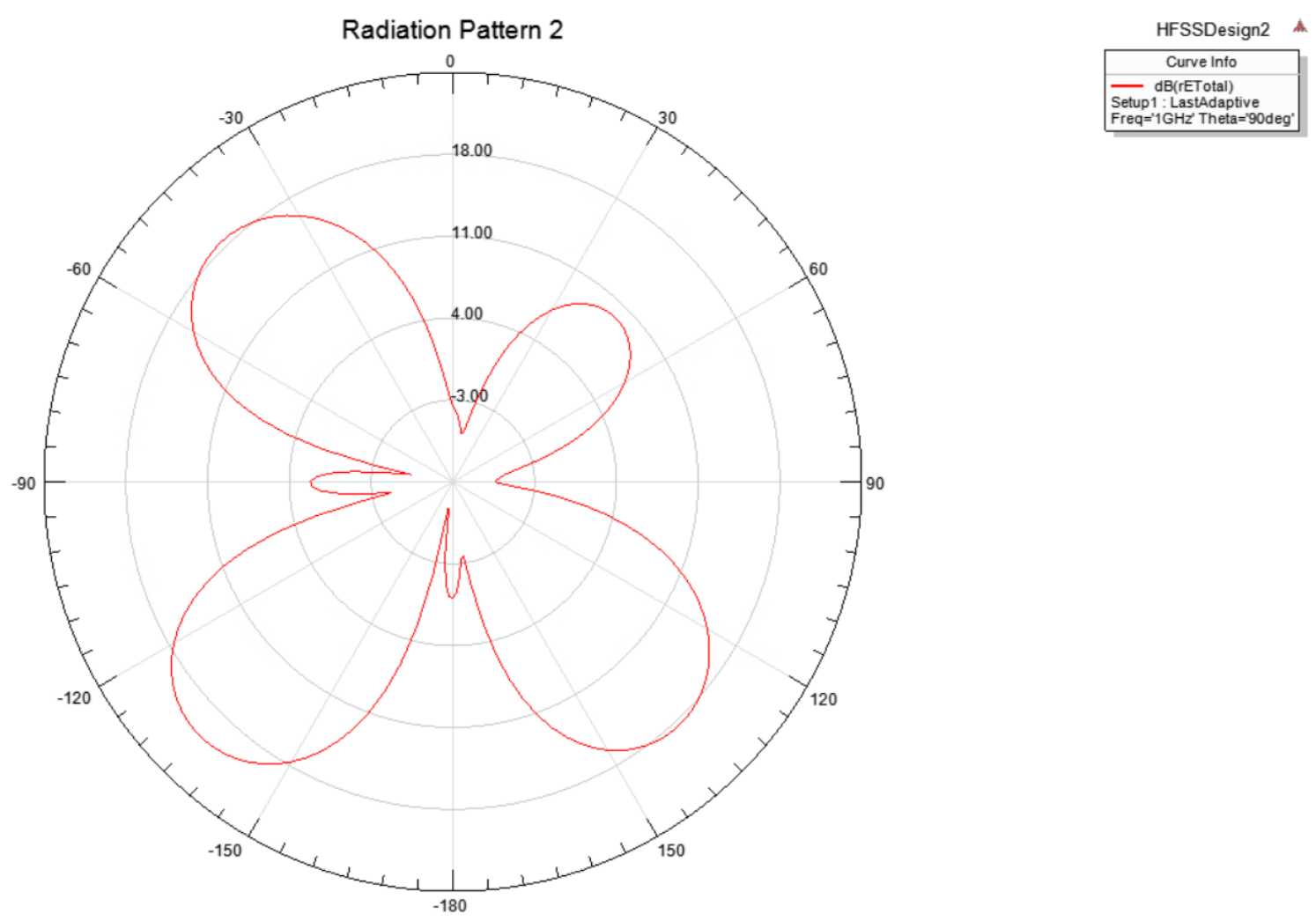

Figure 14: $1 \mathrm{GHz}, 0.3 \mathrm{~m}$ legs

In free space at $1 \mathrm{GHz}$, the wavelength $\lambda=\frac{c}{f}=\frac{3 \times 10^{8} \mathrm{~m} / \mathrm{s}}{1 \times 10^{9} \mathrm{~Hz}}=0.3 \mathrm{~m}$

In Figure 14: $1 \mathrm{GHz}, 0.3 \mathrm{~m}$ legs, a leg length of $0.3 \mathrm{~m}$ was used, or one wavelength at 1 GHz. Therefore, two sidelobes would be expected in this case, which is what Figure 14: 1 $\mathrm{GHz}, 0.3 \mathrm{~m}$ legs shows. Given the results of the 3 tests, the standard method of finding the number of sidelobes appears to be valid. This means that a leg length of $12.8 \mathrm{~cm}$ or less should produce no sidelobes (since at $1 \mathrm{GHz}$, half a wavelength is $0.15 \mathrm{~m}$ ). Likewise, for the upper band the rhombics should not have a leg length larger than $2.5 \mathrm{~cm}$ since that is half of the wavelength in free space at $6 \mathrm{GHz}$. 


\subsubsection{Radiation Pattern Simulations}

Since the radiation pattern is the most important characteristic of the rhombic's design for amplitude comparison monopulse, this was dealt with before gain and S11 simulations. With the size constraints $(32.4 \mathrm{~cm} \times 19.4 \mathrm{~cm})$ the maximum leg length for the rhombics is approximately $12.8 \mathrm{~cm}$. With a known maximum rhombic leg size, this is a starting point for the lower frequency $(0.6-1 \mathrm{GHz})$ radiation pattern simulations. For the higher frequency $(1-6 \mathrm{GHz})$ radiation pattern simulations, the rhombic leg length will start in the $\mathrm{mm}$ size range and increase if necessary, while not increasing beyond $2.5 \mathrm{~cm}$. The first simulations were done at the upper frequency range. Those simulations are shown below: 


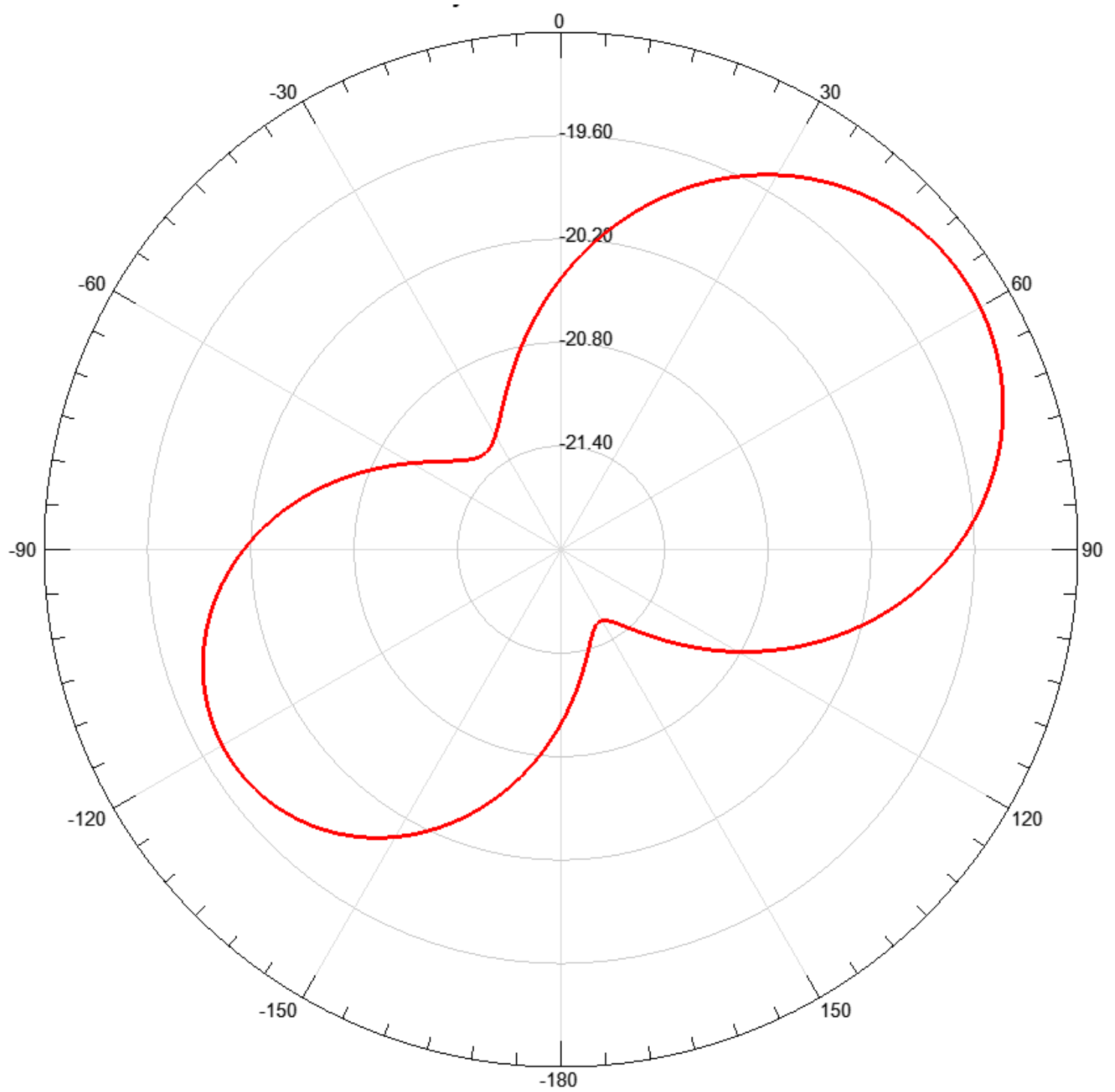

Figure 15: $5 \mathrm{~mm}$ leg length at $6 \mathrm{GHz}$ with $2 \mathrm{~mm}$ wire thickness and $1 \mathrm{~mm}$ separation

The first simulation that was performed is shown above. It was performed at $6 \mathrm{GHz}$ with a $5 \mathrm{~mm}$ leg length. With the wire thickness and separation that was used, the leg length can't be much smaller than $5 \mathrm{~mm}$, so $5 \mathrm{~mm}$ was used as a starting point. The next simulation was done at $5 \mathrm{GHz}$ with the same leg length to confirm that the received signal level is lower as would be expected. 


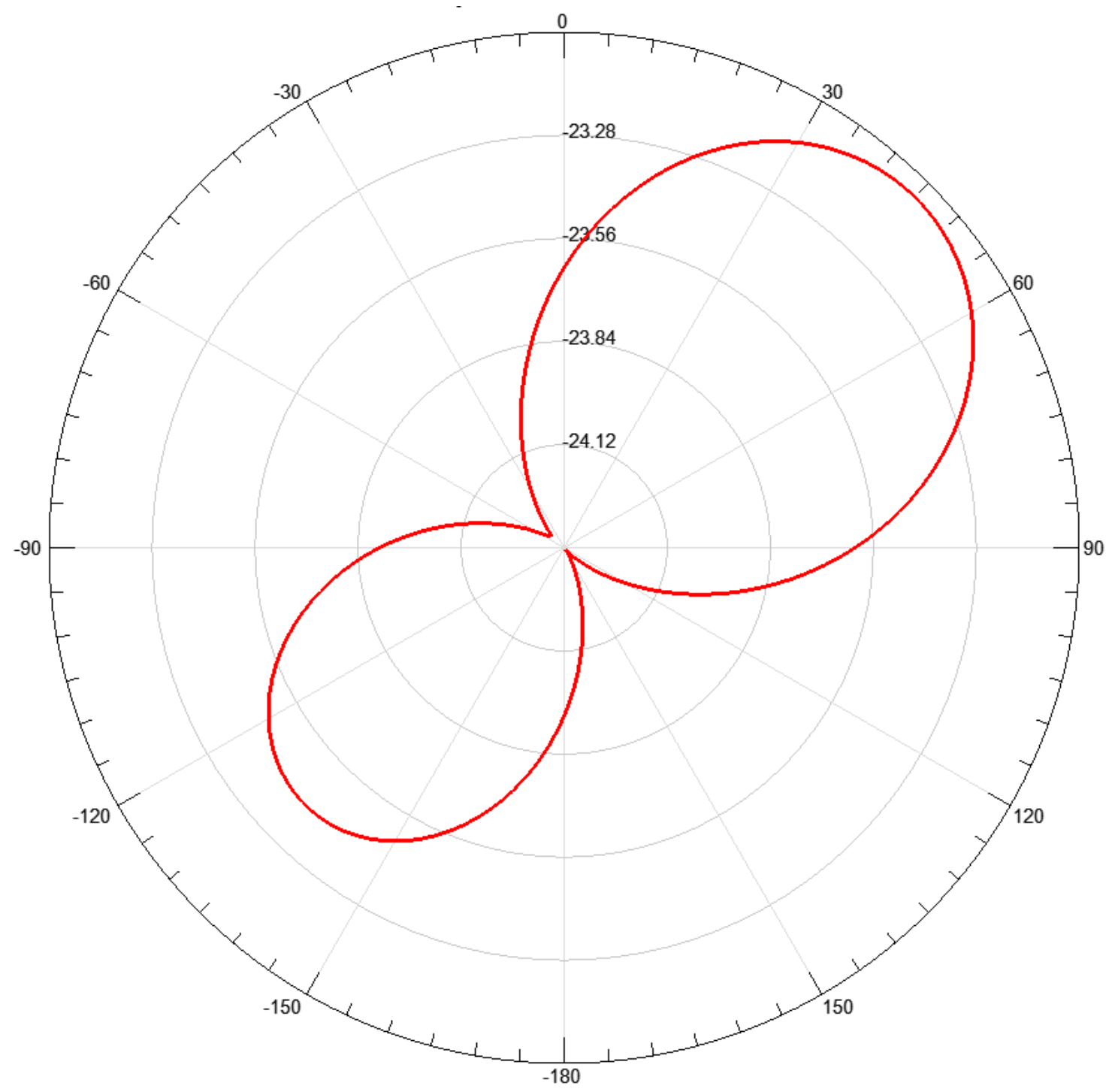

Figure 16: $5 \mathrm{~mm}$ leg length at $5 \mathrm{GHz}$ with $2 \mathrm{~mm}$ wire thickness and $1 \mathrm{~mm}$ separation

As expected, the received signal level is lower than the $6 \mathrm{GHz}$ simulation. Since this is true, a larger rhombic will be needed in order to get a more reasonable received power. The next few simulations will show increasing rhombic sizes to find the point where the received signal strength can't increase (while keeping the general shape of the radiation pattern shown in Figure 17: $1.2 \mathrm{~cm}$ leg length at $3.5 \mathrm{GHz}$ with $2 \mathrm{~mm}$ wire thickness and 1 $\mathrm{mm}$ separation). This is done at $3.5 \mathrm{GHz}$ since it is in the middle of the higher end frequency range. 


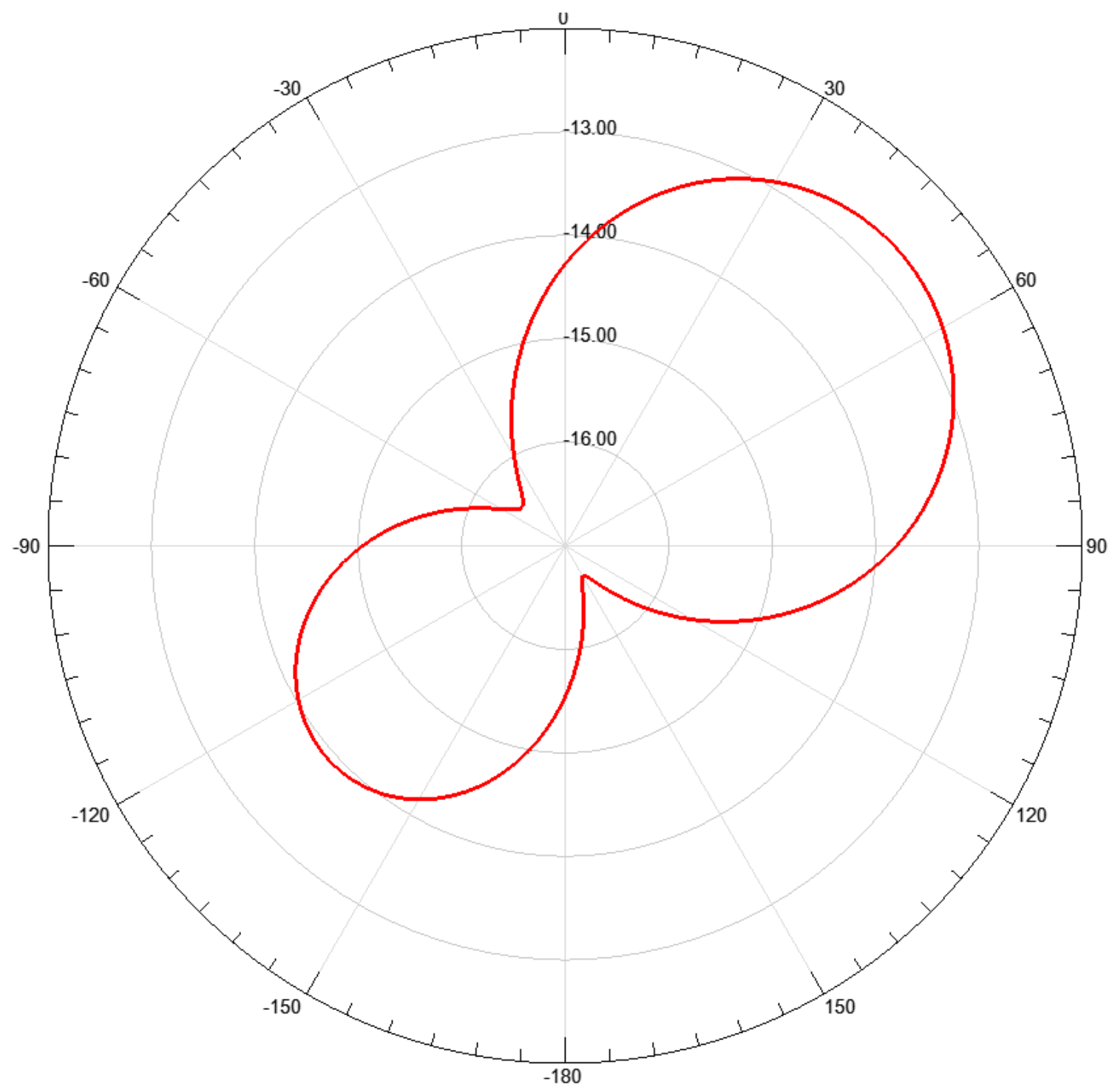

Figure 17: $1.2 \mathrm{~cm}$ leg length at $3.5 \mathrm{GHz}$ with $2 \mathrm{~mm}$ wire thickness and $1 \mathrm{~mm}$ separation

With the leg length being increased to $1.2 \mathrm{~cm}$ it can be seen that the received power level and the shape of the radiation pattern improve significantly. For this reason, the size was increased again to see if the pattern would improve further. This is shown in the following three figures. 


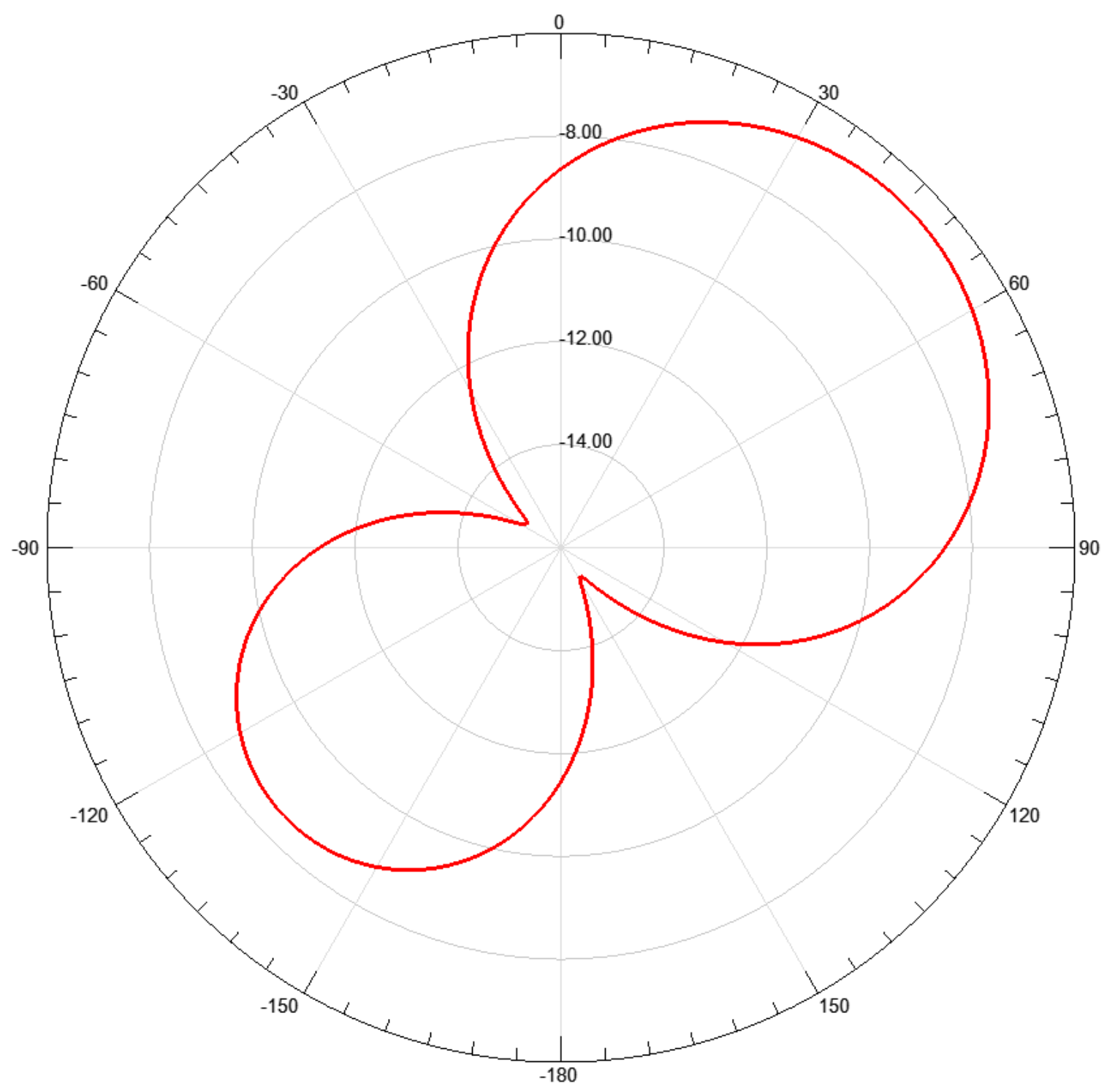

Figure 18: $1.8 \mathrm{~cm}$ leg length at $3.5 \mathrm{GHz}$ with $2 \mathrm{~mm}$ wire thickness and $1 \mathrm{~mm}$ separation 


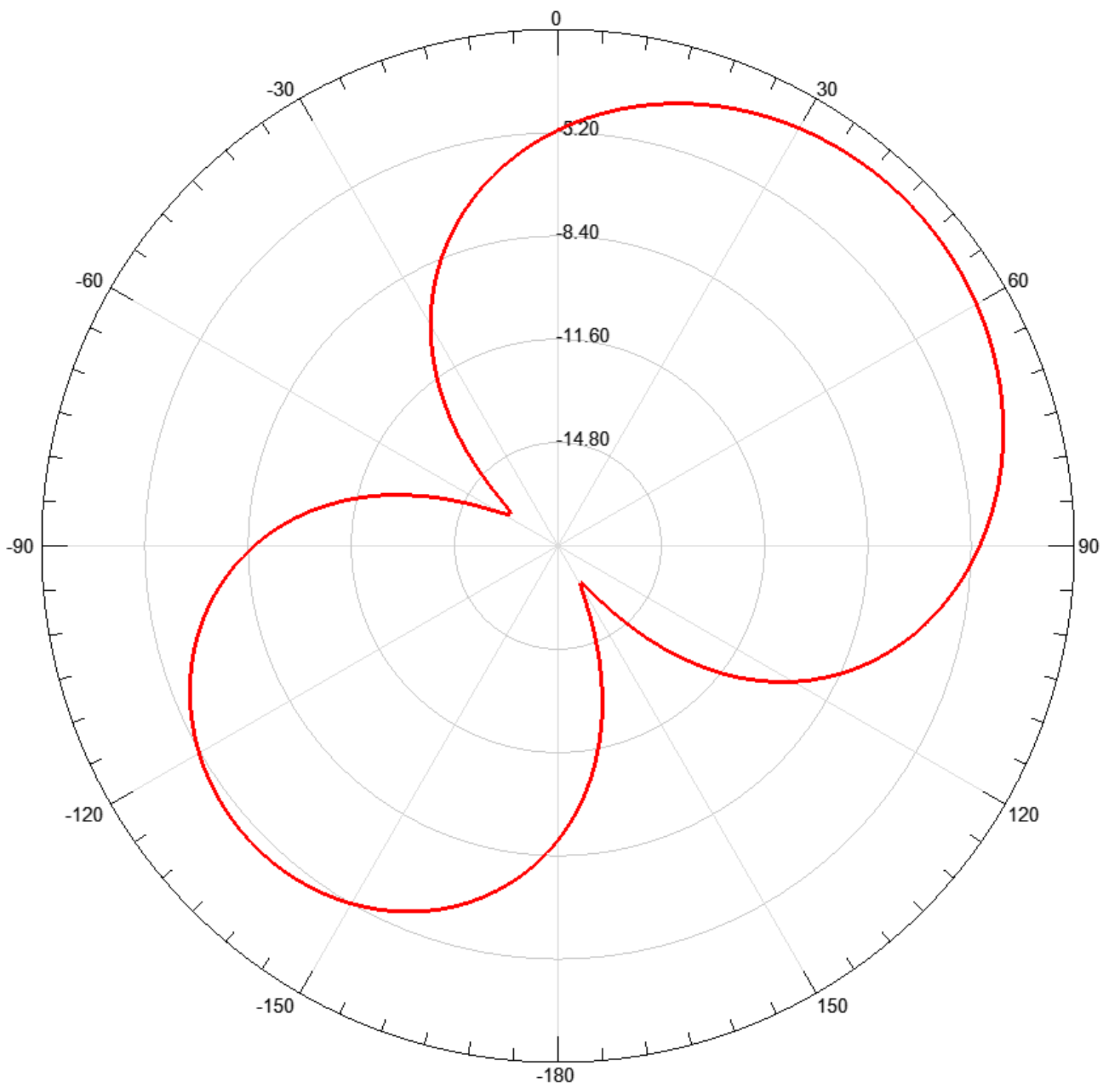

Figure 19: $2.2 \mathrm{~cm}$ leg length at $3.5 \mathrm{GHz}$ with $2 \mathrm{~mm}$ wire thickness and $1 \mathrm{~mm}$ separation 


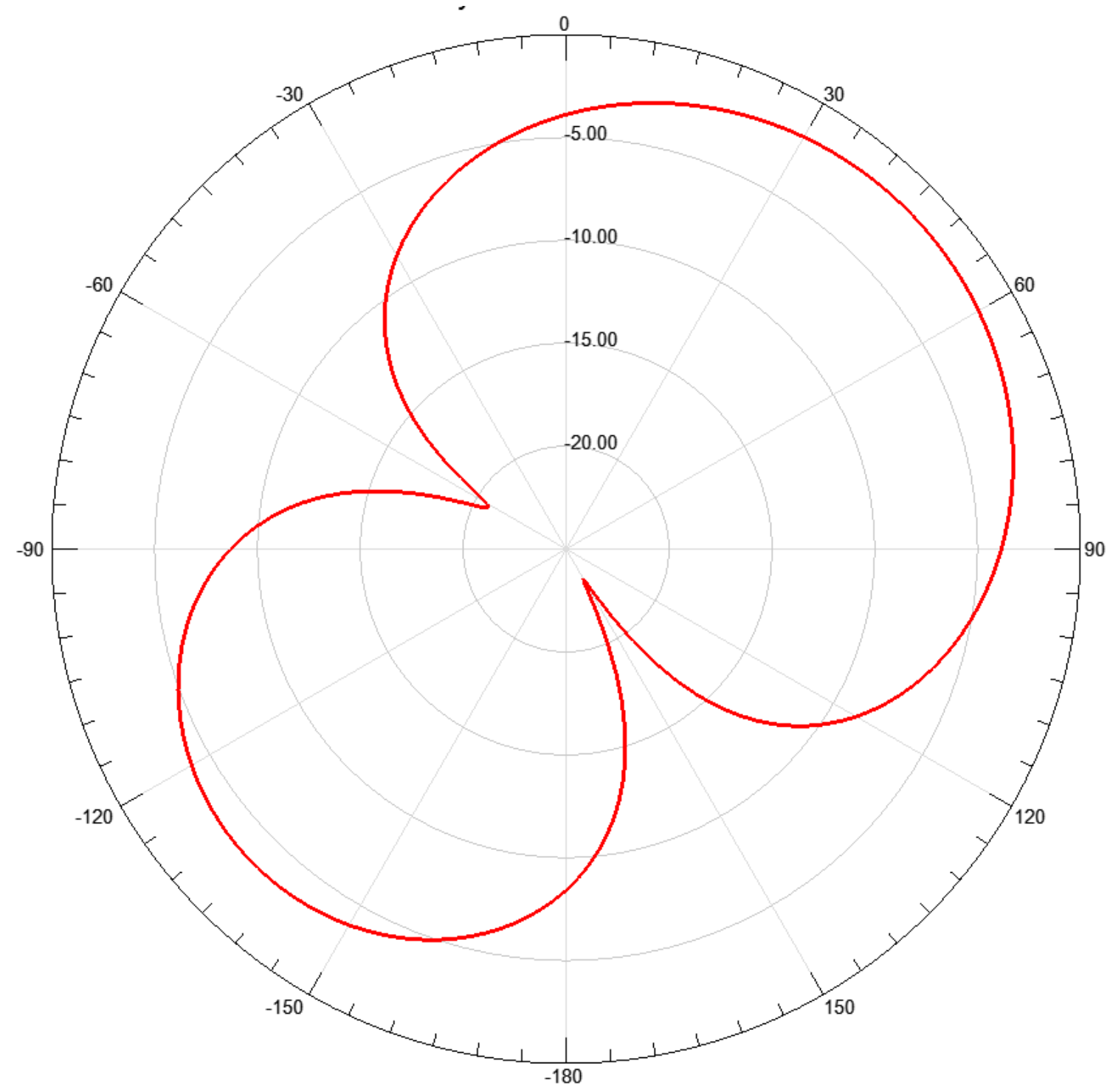

Figure 20: $2.5 \mathrm{~cm}$ leg length at $3.5 \mathrm{GHz}$ with $2 \mathrm{~mm}$ wire thickness and $1 \mathrm{~mm}$ separation The rhombics appear to have an increasing HPBW and received power as the leg length increases. However, the leg length should not be any higher than $2.5 \mathrm{~cm}$ to avoid sidelobes around $6 \mathrm{GHz}$, so the leg length was reduced to $2.3 \mathrm{~cm}$ to see if the radiation pattern can be improved with a leg length slightly smaller than $2.5 \mathrm{~cm}$. 


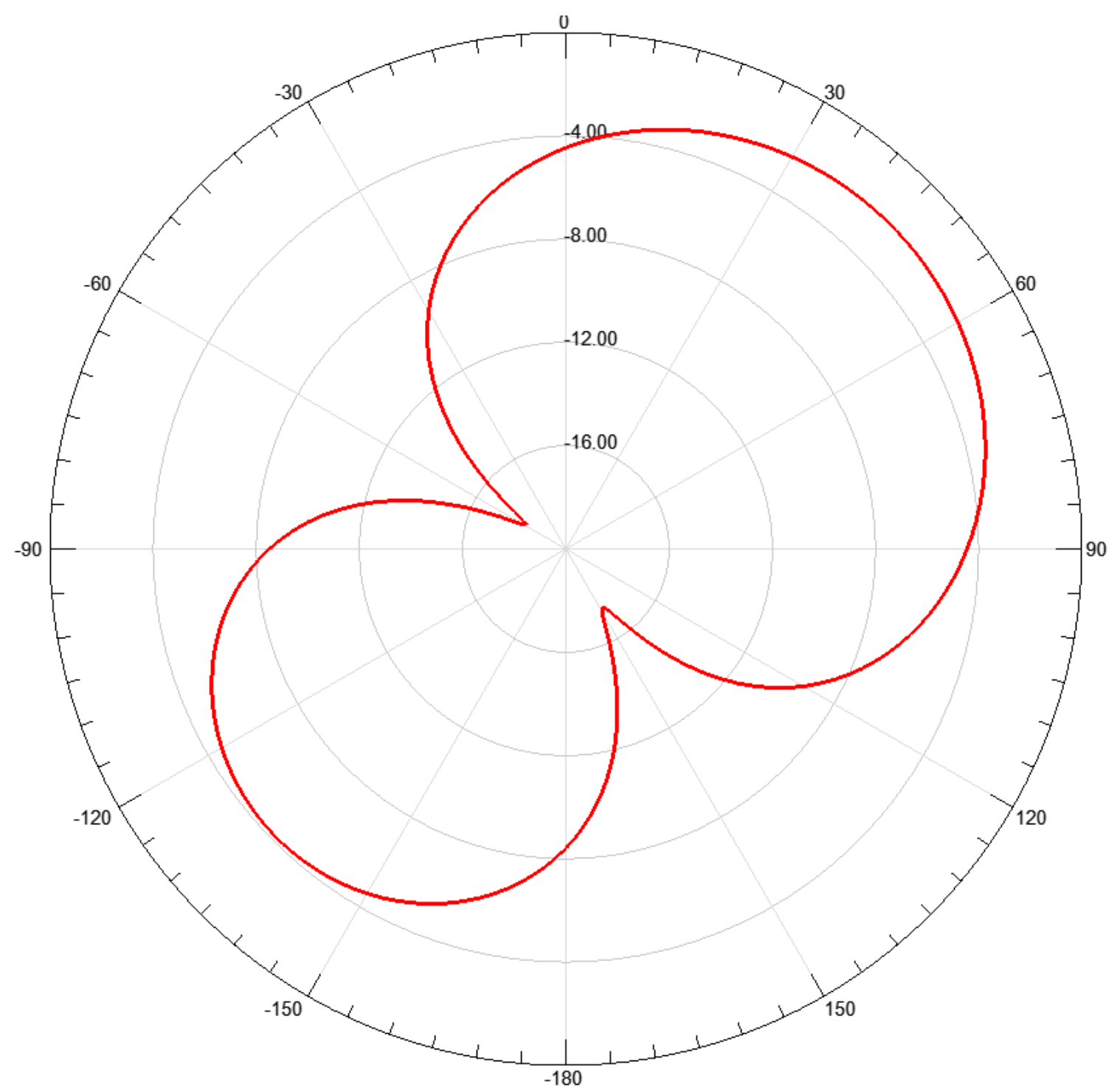

Figure 21: $2.3 \mathrm{~cm}$ leg length at $3.5 \mathrm{GHz}$ with $2 \mathrm{~mm}$ wire thickness and $1 \mathrm{~mm}$ separation

In Figure 21: $2.3 \mathrm{~cm}$ leg length at $3.5 \mathrm{GHz}$ with $2 \mathrm{~mm}$ wire thickness and $1 \mathrm{~mm}$ separation it can be seen that the $2.3 \mathrm{~cm}$ leg length has a better received signal and pattern than the $2.5 \mathrm{~cm}$ and $2.2 \mathrm{~cm}$ simulations. This appears to be a good starting point for testing the rhombic antennas in the higher frequency range. Simulations ranging from 1-6 GHz with this leg length are shown in Appendix A (note that the results in appendices $\mathrm{A}$ and $\mathrm{B}$ reflect the radiation patterns after the gain simulations were 
performed). These simulations show that this leg length is sufficient for the upper frequency range for this thesis.

For the lower frequency range, the maximum leg length that can be used is $12.8 \mathrm{~cm}$. Therefore, the lower frequency simulations shown below were done at the maximum leg length and reduced from there. Using the same logic as for the higher frequency range, the initial simulations for the lower range were performed at the mid-point frequency (800 MHz). 


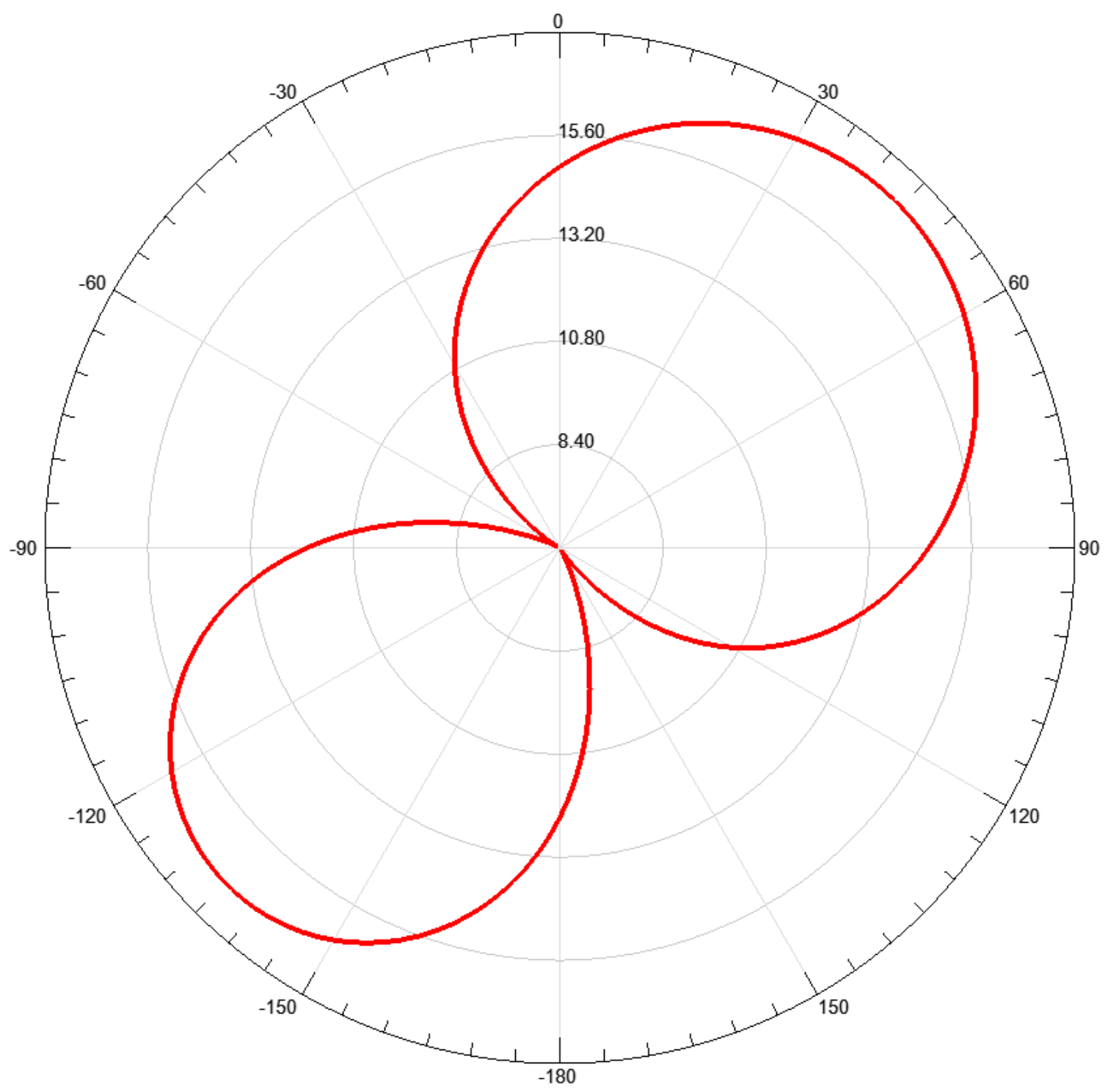

Figure 22: $12.8 \mathrm{~cm}$ leg length at $800 \mathrm{MHz}$ with $2 \mathrm{~mm}$ wire thickness and $1 \mathrm{~mm}$ separation

The approach that was taken for the lower frequency range was similar to the higher frequency range. The main difference being that the longest possible leg length was used for the first radiation pattern simulation shown above. Subsequently simulations were performed with smaller leg lengths until the ideal leg length was found. 


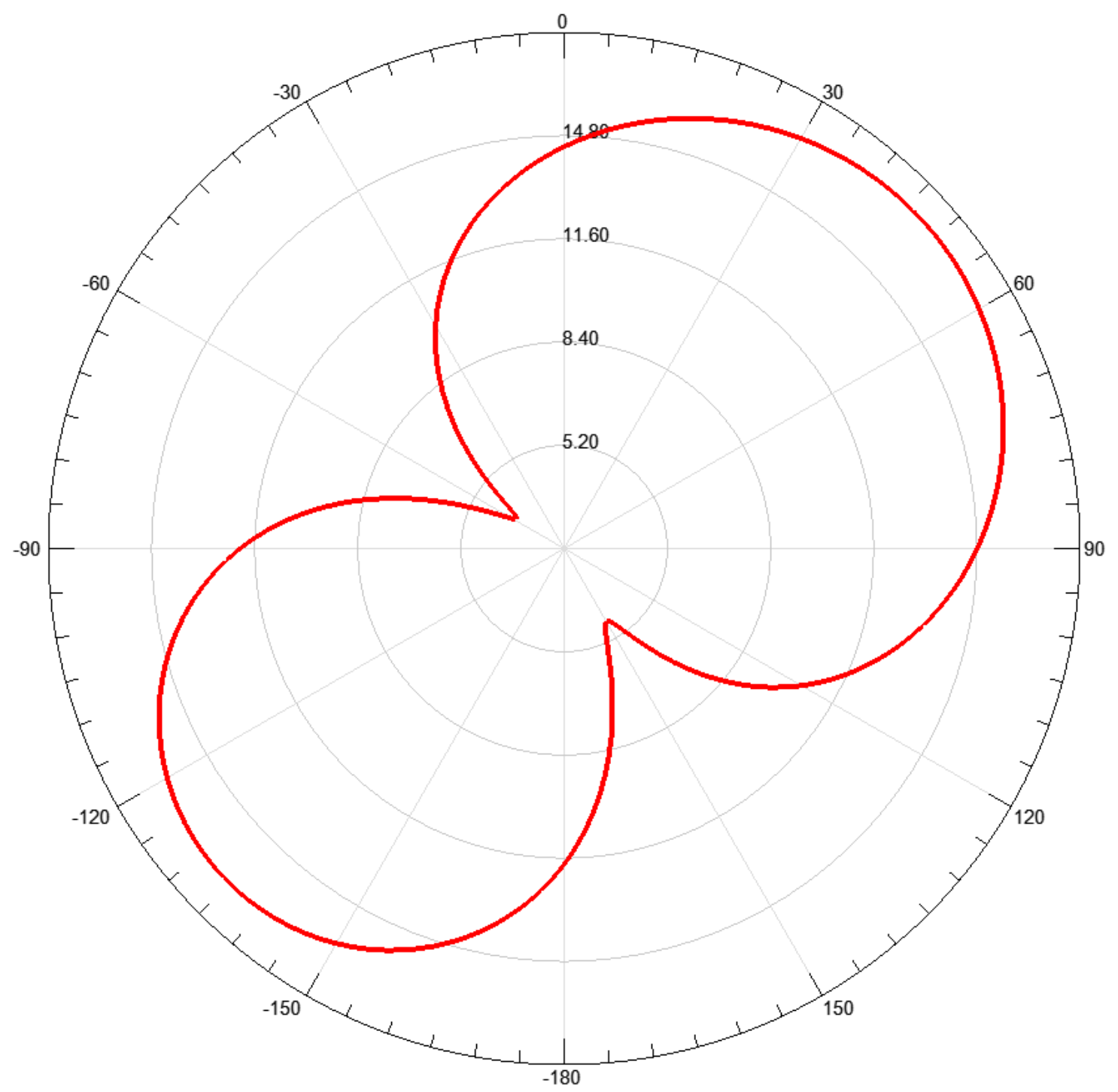

Figure 23: $12.5 \mathrm{~cm}$ leg length at $800 \mathrm{MHz}$ with $2 \mathrm{~mm}$ wire thickness and $1 \mathrm{~mm}$ separation

In Figure 23: $12.5 \mathrm{~cm}$ leg length at $800 \mathrm{MHz}$ with $2 \mathrm{~mm}$ wire thickness and $1 \mathrm{~mm}$ separation, both the shape of the radiation pattern and the received signal strength was slightly worse with a $12.5 \mathrm{~cm}$ leg length. Therefore, the size is increased to see if there is a further improvement in the radiation pattern and received signal strength when compared to the $12.8 \mathrm{~cm}$ leg length. 


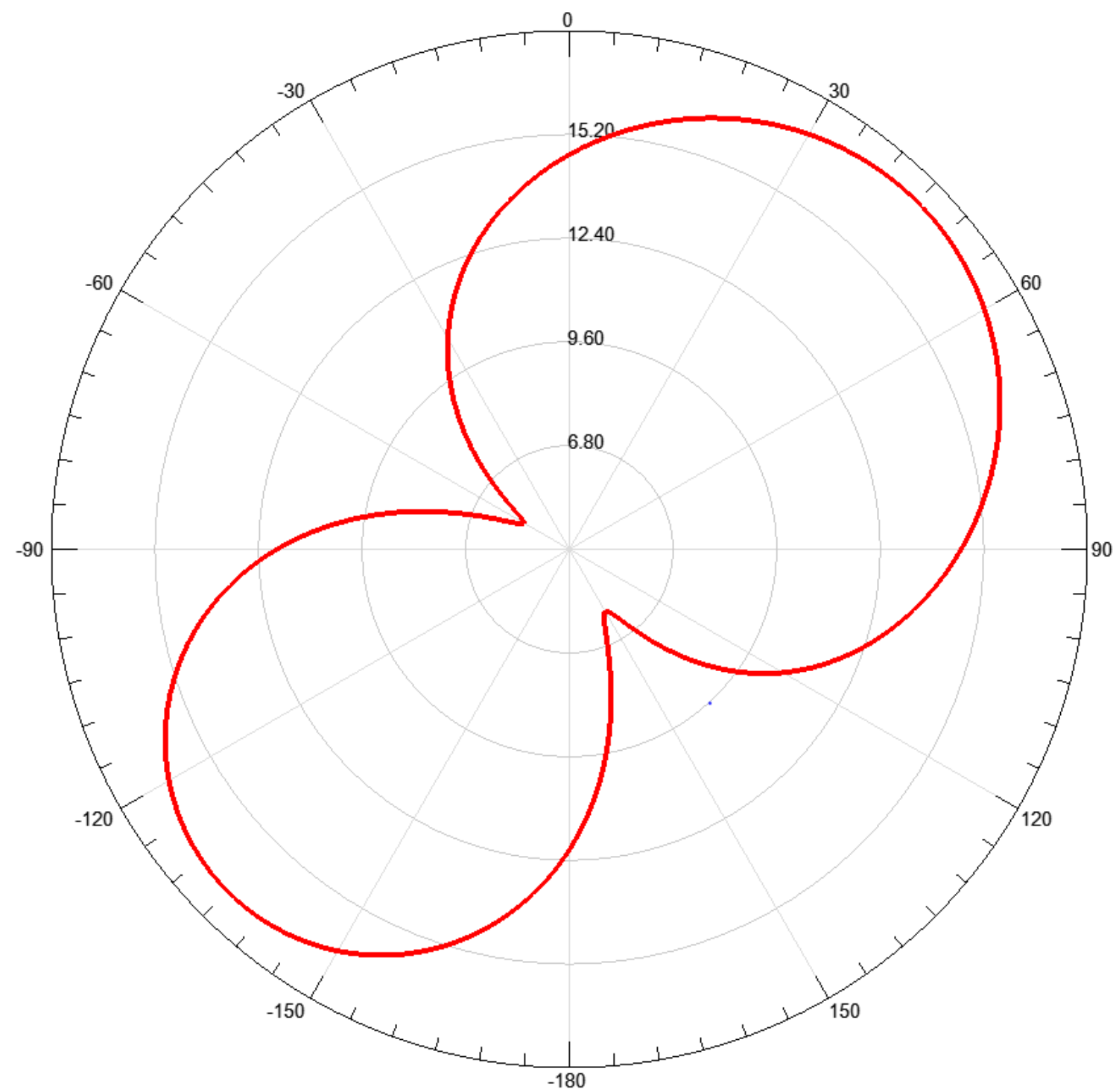

Figure 24: $12.7 \mathrm{~cm}$ leg length at $800 \mathrm{MHz}$ with $2 \mathrm{~mm}$ wire thickness and $1 \mathrm{~mm}$ separation

In the two previous figures, the $12.5 \mathrm{~cm}$ and $12.7 \mathrm{~cm}$ leg lengths have slightly worse radiation patterns and/or received powers than the $12.8 \mathrm{~cm}$ leg length. For this reason, the $12.8 \mathrm{~cm}$ leg length is used for the lower band. The simulations for the other frequencies in the lower band are shown in Appendix B. 


\subsubsection{S11 Simulations}

Now that the ideal leg length has been found for a $600 \Omega$ terminating resistance, the ideal S11 needs to be found. S11 is the reflection coefficient of an antenna, which represents the amount of power reflected from the antenna. If the $\mathrm{S} 11$ of an antenna were $0 \mathrm{~dB}$, all of the power is reflected from the antenna, therefore no power is radiated [16]. As the S11 decreases below $0 \mathrm{~dB}$, more power is radiated by the antenna and less is reflected.

This was tested for both the upper and lower frequency bands iterating on the initial terminating resistance of $600 \Omega$. The S11 simulations for the upper band were performed from 1-6 GHz, however the lower band simulations were performed from $0.6-2 \mathrm{GHz}$. This is because it is not known if the S11 is better for the rhombic designed for the upper or lower band near $1 \mathrm{GHz}$. The first S11 simulation is shown below, which uses the 12.8 $\mathrm{cm}$ leg length rhombic and a $600 \Omega$ terminating resistance.

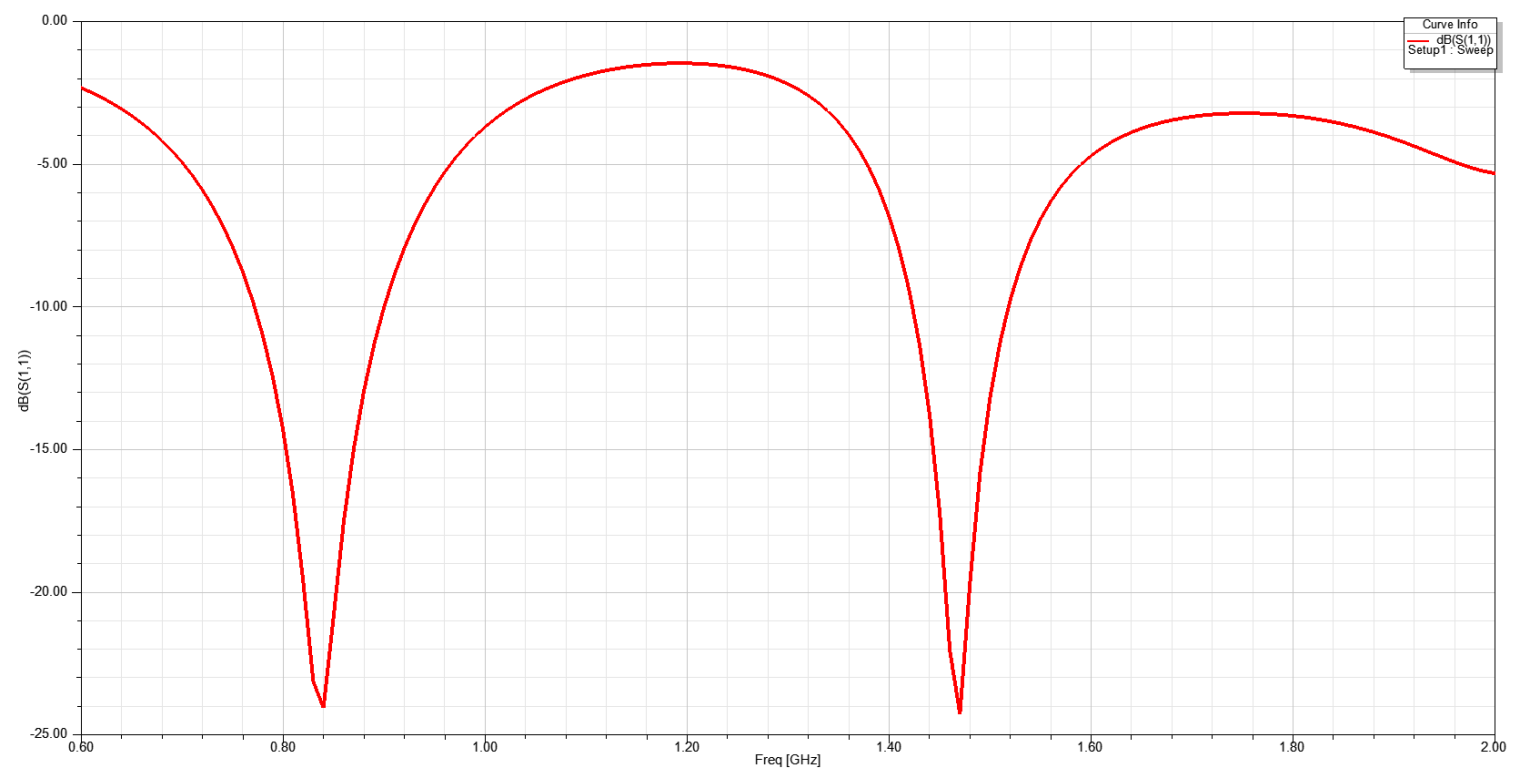

Figure 25: S11 for $12.8 \mathrm{~cm}$ leg length rhombic with a $600 \Omega$ terminating resistance

In Figure 25: S11 for $12.8 \mathrm{~cm}$ leg length rhombic with a $600 \Omega$ terminating resistance, the S11 performance is poor for a large portion of the simulated $0.6-2 \mathrm{GHz}$ frequency 
range. With a rhombic (since it is a wire antenna) the S11 should ideally be below $-8 \mathrm{~dB}$ [17]. This is expected since the frequency range is large. The S11 was iterated upon in the following simulations. The following simulations both increase and decrease the terminating resistance to show the effect of the terminating resistance on the S11 of a rhombic antenna for the frequencies of interest.

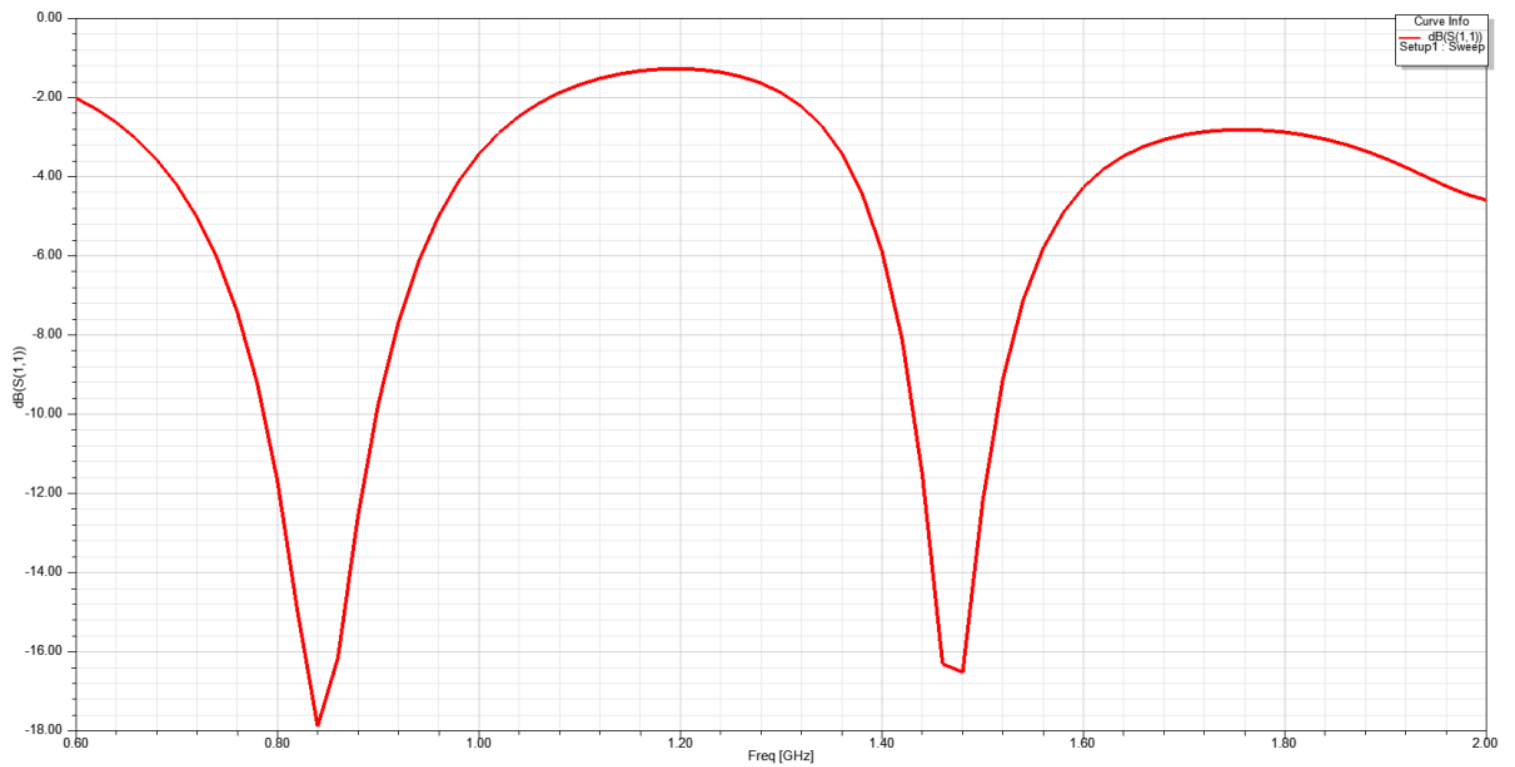

Figure 26: $S 11$ for $12.8 \mathrm{~cm}$ leg length rhombic with a $700 \Omega$ terminating resistance

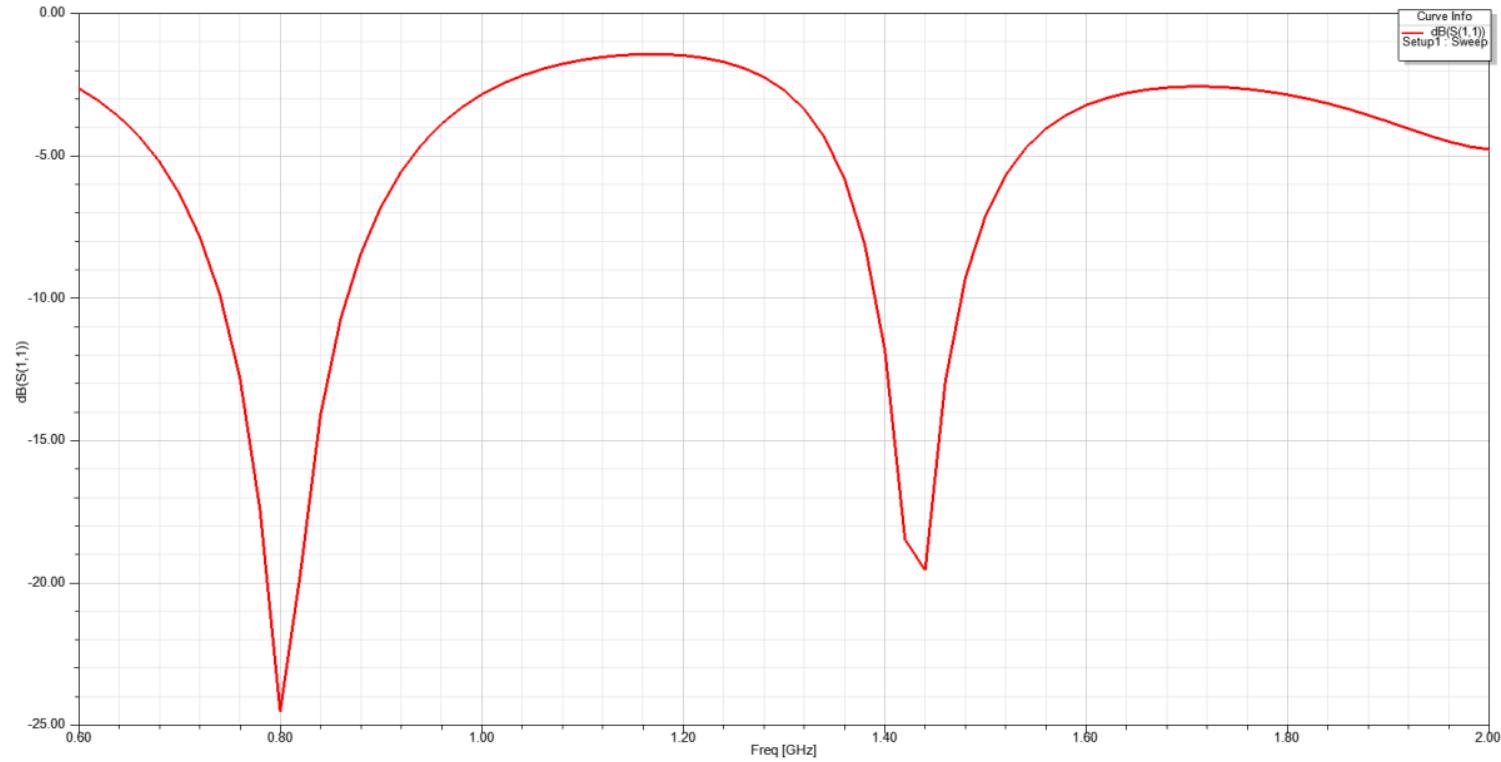

Figure 27: S11 for $12.8 \mathrm{~cm}$ leg length rhombic with a $500 \Omega$ terminating resistance 
It can be seen that both increasing and decreasing the terminating resistance for the 12.8 $\mathrm{cm}$ leg length rhombic is detrimental to the $\mathrm{S} 11$ of the lower band rhombic. Therefore, a $600 \Omega$ terminating resistance is used in the upcoming gain section.

The next set of simulations were performed for the upper frequency range (1-6 GHz). Similar to the lower band simulations, a terminating resistance of $600 \Omega$ was simulated first. However, only terminating resistances smaller than $600 \Omega$ were tested.

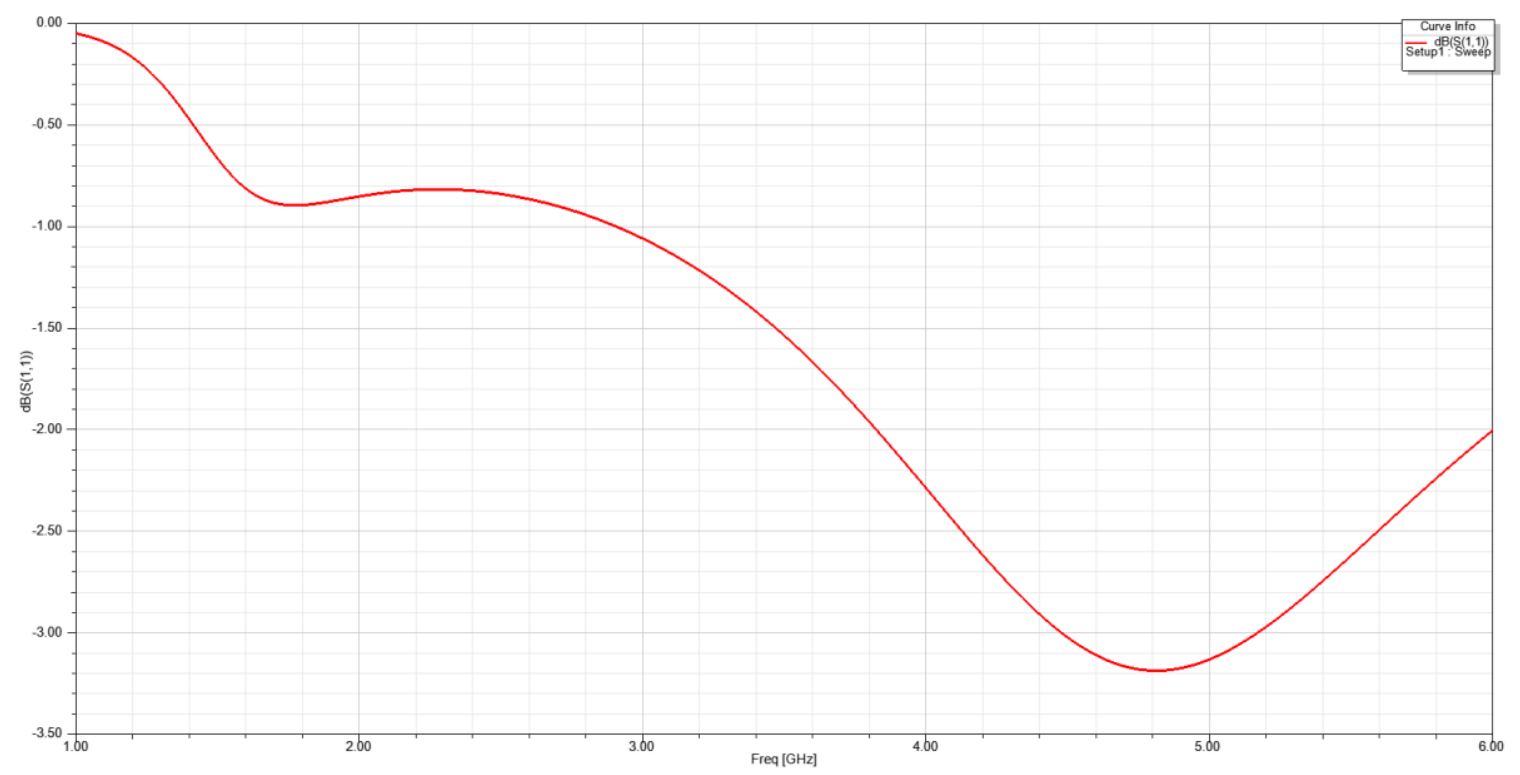

Figure 28: $S 11$ for $2.3 \mathrm{~cm}$ leg length rhombic with a $600 \Omega$ terminating resistance

Since the S11 results are so poor for the upper band, a far lower terminating resistance of $50 \Omega$ is tested in Figure 29: S11 for $2.3 \mathrm{~cm}$ leg length rhombic with a $50 \Omega$ terminating resistance. 


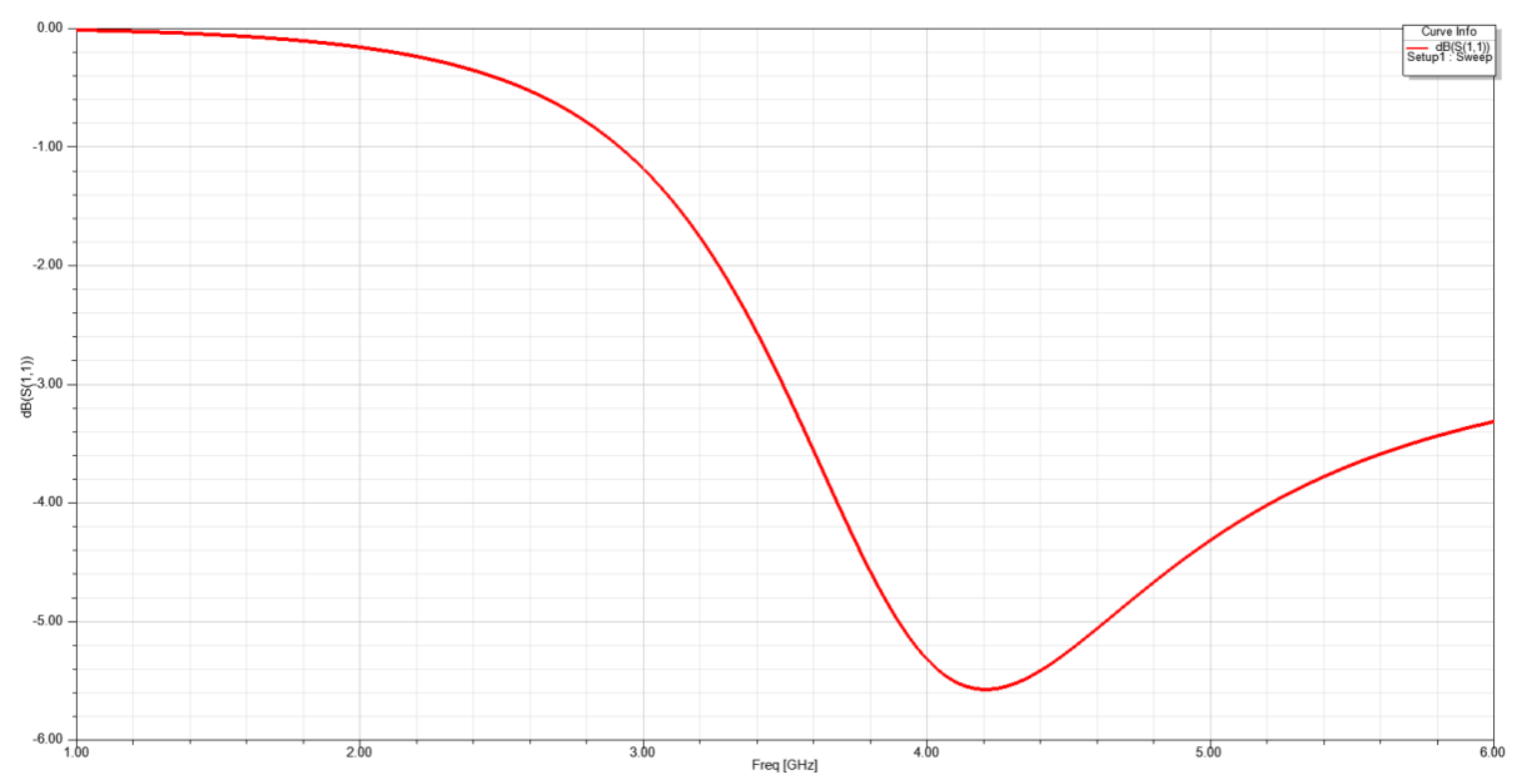

Figure 29: S11 for $2.3 \mathrm{~cm}$ leg length rhombic with a $50 \Omega$ terminating resistance

Using a far smaller terminating resistance, the S11 results were far better, however they are still significantly worse than the lower band results. Therefore, the terminating resistance was increased to $200 \Omega$ to see if there is a more room for improvement for the S11 of the upper band rhombic.

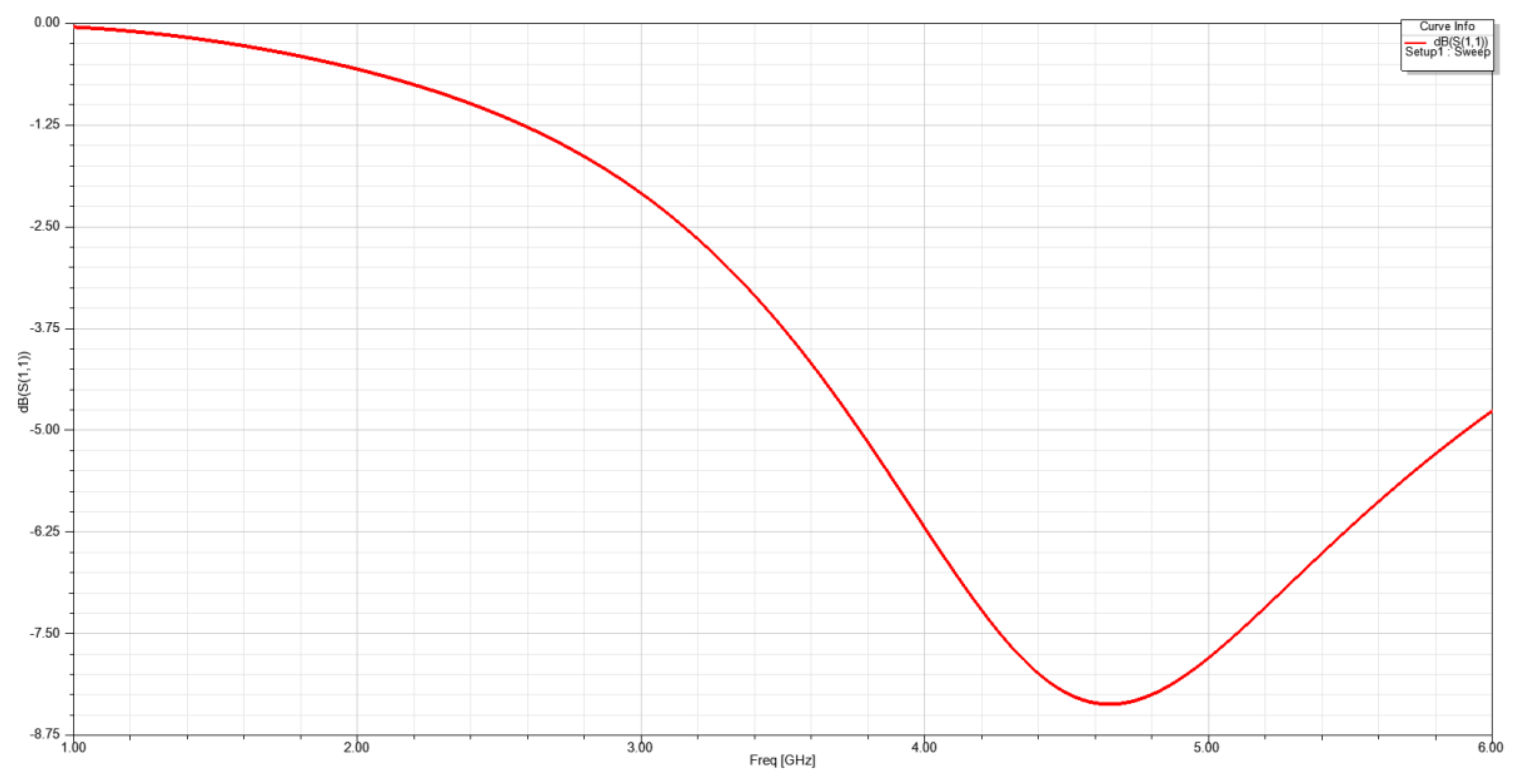

Figure 30: S11 for $2.3 \mathrm{~cm}$ leg length rhombic with a $200 \Omega$ terminating resistance 
Once again, the S11 has improved substantially with the terminating resistance changing from 50 to $200 \Omega$. The terminating resistance was brought down to $100 \Omega$ to see if there was a further improvement on the S11 of the rhombic.

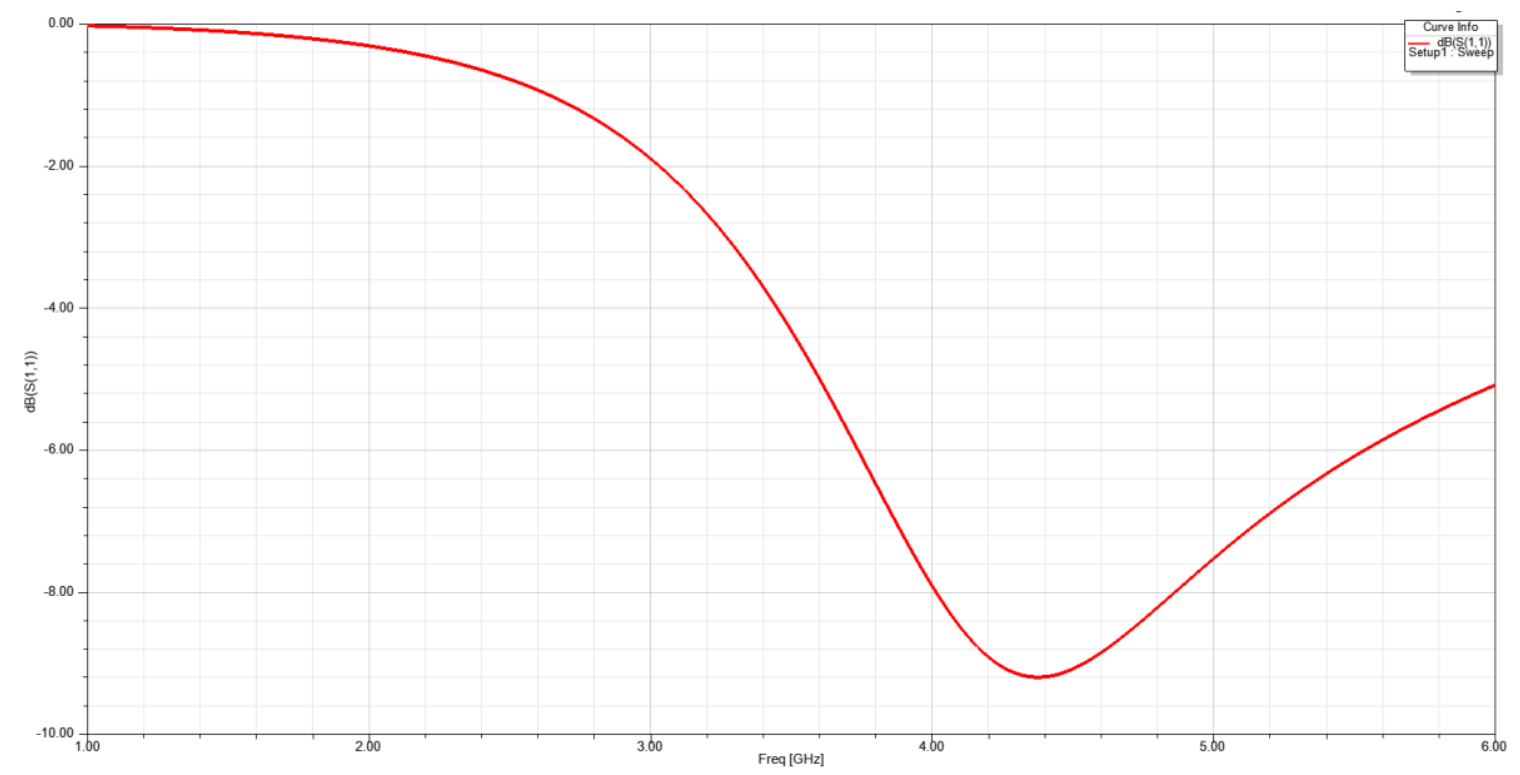

Figure 31: $S 11$ for $2.3 \mathrm{~cm}$ leg length rhombic with a $100 \Omega$ terminating resistance

Somewhere around a $100 \Omega$ terminating resistance appears to be the best performance for the upper band rhombic in terms of S11. With this terminating resistance and the leg length of $2.3 \mathrm{~cm}$ found earlier, the gain simulations will be performed along with the 12.8 $\mathrm{cm}$ leg length rhombic with a $600 \Omega$ terminating resistance. Since the S11 of the upper band rhombic is so poor from $1-2 \mathrm{GHz}$, the lower band rhombic will be used up to 2 GHz. This means that the upper band rhombic will be used from $2-6 \mathrm{GHz}$. While the S11 characteristics of these rhombics are not ideal over a large portion of their respective operating frequencies, they do receive power at each point in the frequency domain.

The S11 characteristics of the rhombics could be improved significantly if a larger UAV is used. In the S11 figures from both the upper and lower frequency ranges, the results are much better over an octave in frequency. This means that if a UAV is chosen that could 
carry three sets of two rhombics, then new frequency ranges that are approximately an octave could improve the S11 of the rhombic antennas. The new frequency ranges could be $0.6-1.2 \mathrm{GHz}, 1.2-2.4 \mathrm{GHz}$, and $2.4-6 \mathrm{GHz}$ for example. This would still not provide ideal S11 characteristics, but it would improve the S11 of the rhombics significantly.

Since the lower band rhombic is now being used up to $2 \mathrm{GHz}$ it must be resized to avoid sidelobes up to $2 \mathrm{GHz}$. Following the same process that was used in section 4.3.2, the optimal leg length for the radiation pattern was found to be $6 \mathrm{~cm}$. The $\mathrm{S} 11$ plot for this rhombic is given below.

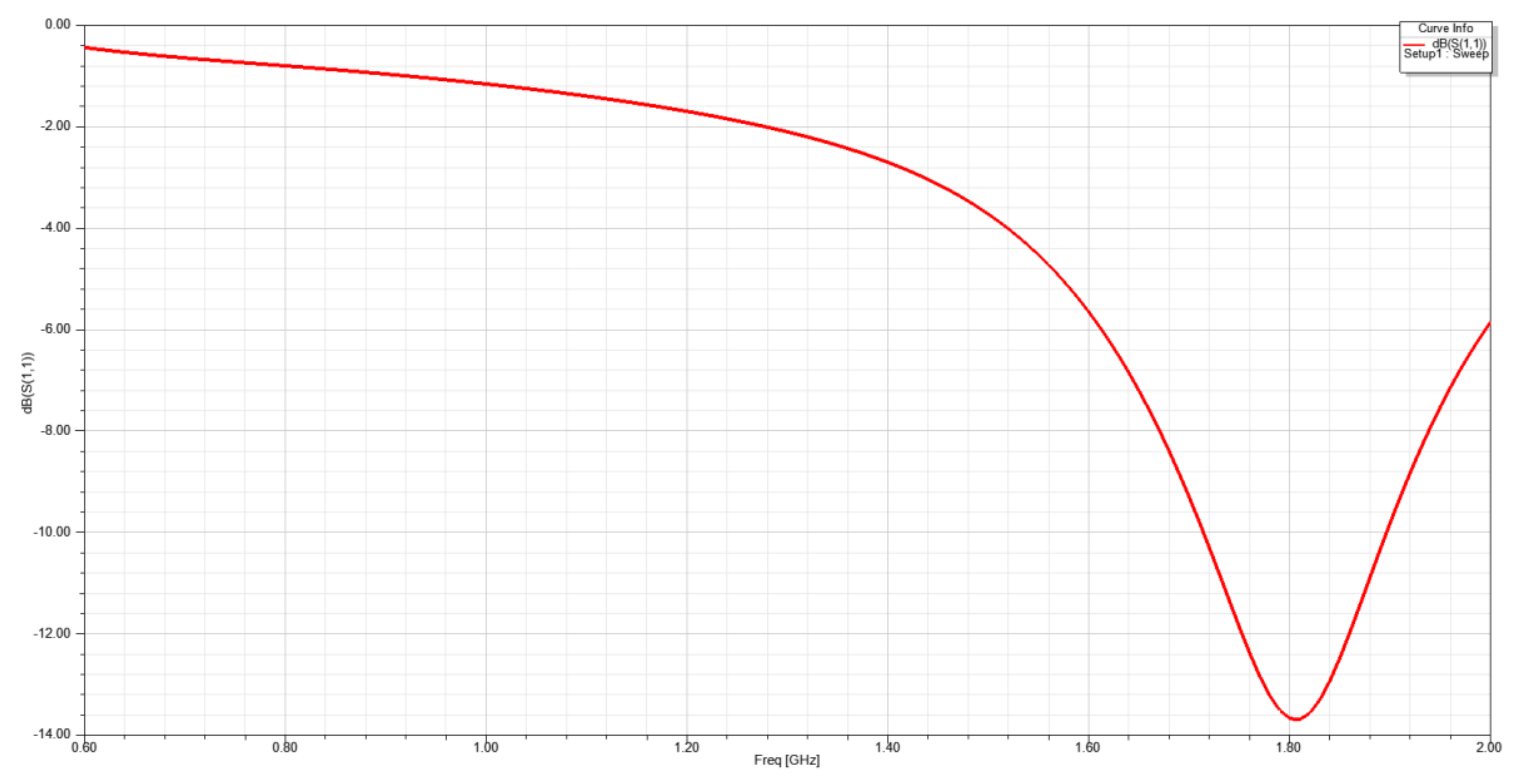

Figure 32: $S 11$ for $6 \mathrm{~cm}$ leg length rhombic with a $600 \Omega$ terminating resistance

In terms of $\mathrm{S} 11$, the $6 \mathrm{~cm}$ leg length rhombic provides worse performance from 0.6-1 $\mathrm{GHz}$, but far better performance from 1-6 GHz when compared to the upper band rhombic. 


\subsubsection{Gain Simulations}

Now that a reasonable radiation pattern has been simulated for each frequency range, the boresight gain will be optimized for the radiation pattern. Since the upper and lower frequency ranges are so large, it is unrealistic to try to make the gain flat over a $0.6-6$ $\mathrm{GHz}$ range. Therefore, the focus was to make the gain as large as possible without negatively affecting the S11 or the radiation pattern too much. This was done by iterating on the rhombic leg length close to the optimal sizes found in the previous section. The gain simulations for the upper frequency range with leg sizes and related radiation patterns are shown below. Note that from figure to figure the rhombic leg length is changing. This was done to see if there is any improvement in the gain and if that improvement is worth changing the rhombic leg length for. The gain in the y-axis of these figures is the gain of the antenna in $\mathrm{dB}$. Note that for the lower band rhombic, only the $6 \mathrm{~cm}$ rhombic's gain is simulated since higher leg lengths result in unwanted sidelobes near $2 \mathrm{GHz}$ and lower leg lengths result in worse radiation pattern characteristics. 


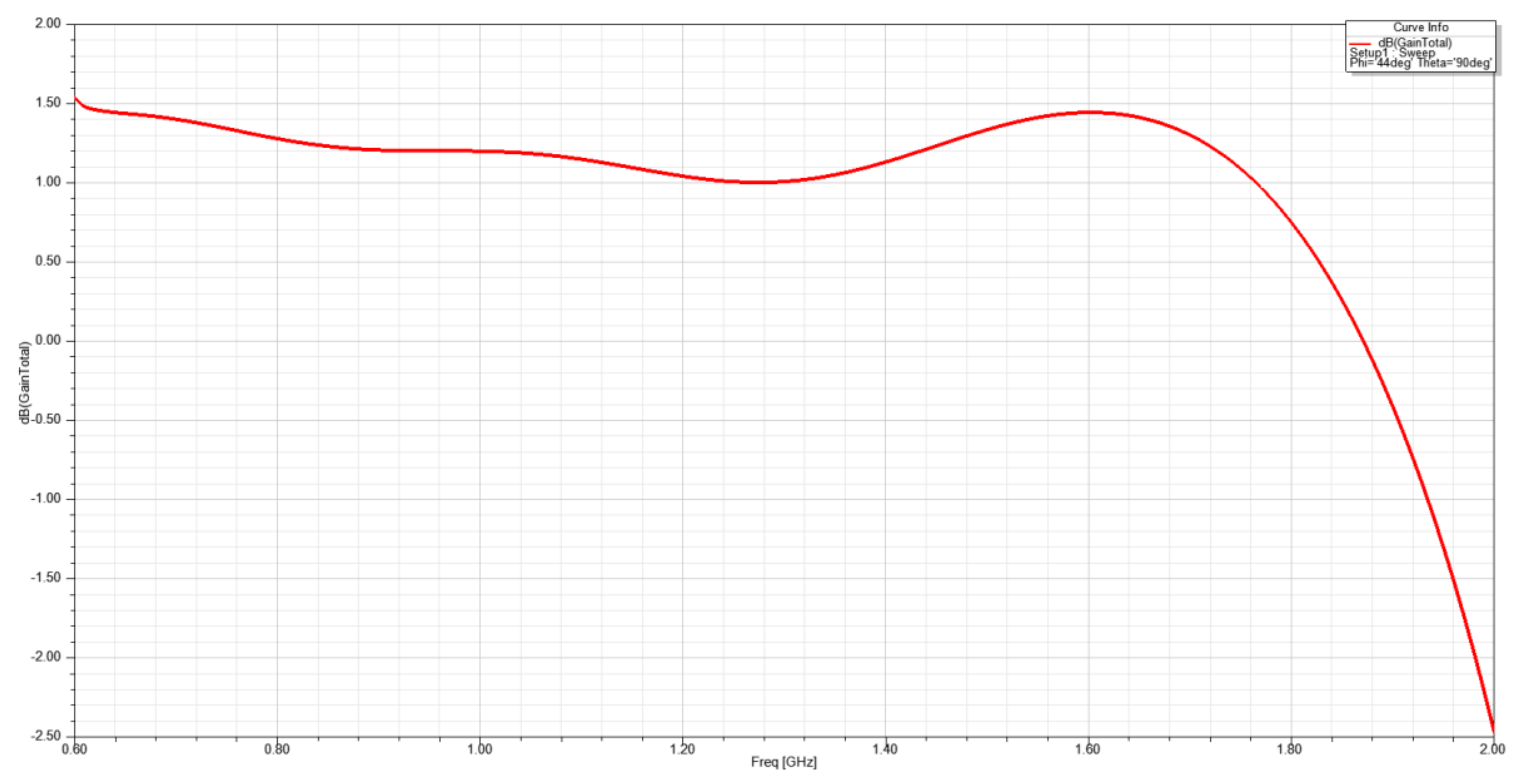

Figure 33: $6 \mathrm{~cm}$ leg length rhombic gain with a $600 \Omega$ terminating resistance

The following simulation results are performed for the upper frequency range rhombic. The first simulation is with a $2.3 \mathrm{~cm}$ leg length and similar to the upper band rhombic, the leg length is changed in order to determine whether or not changes in the leg length result in a positive change in the gain.

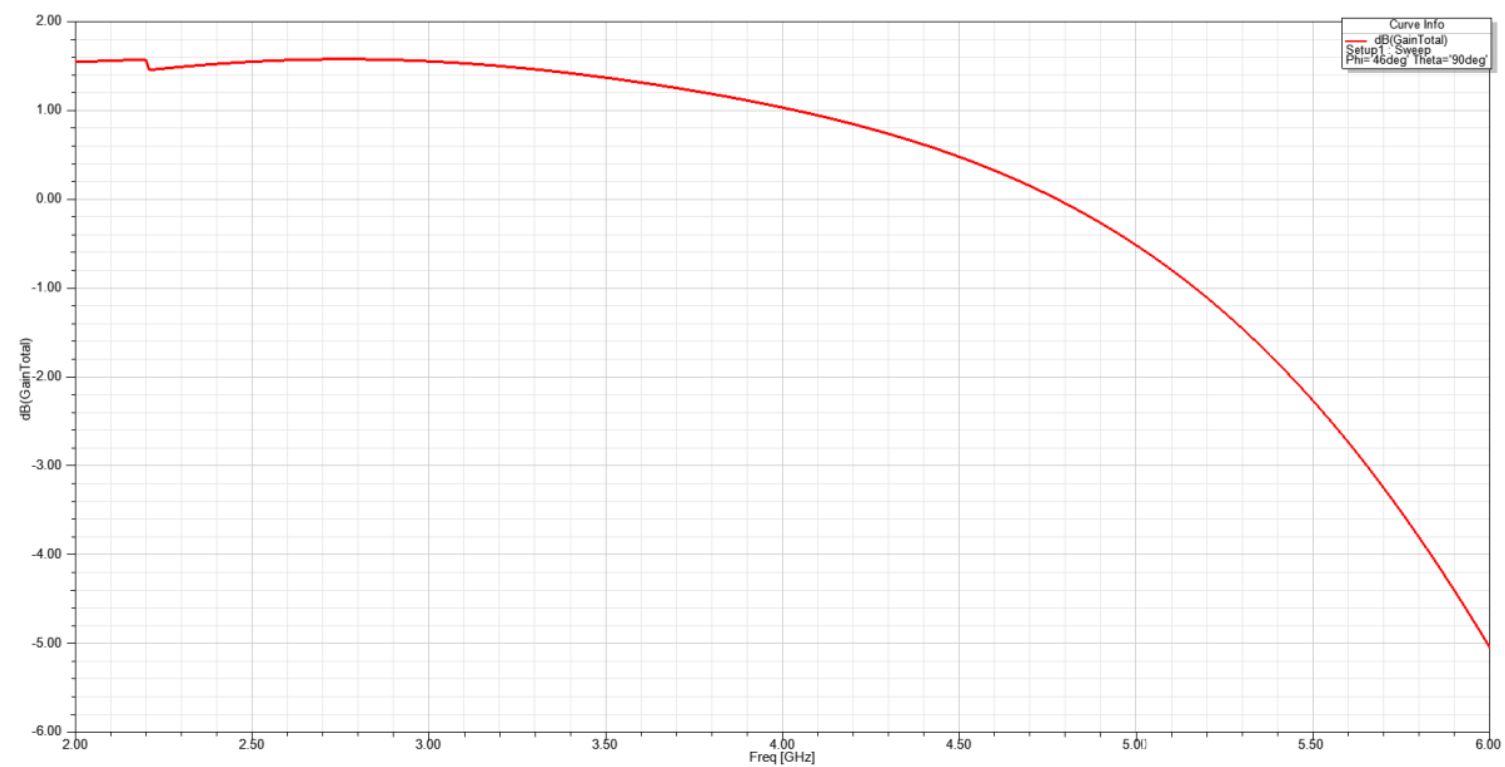

Figure 34: $2.3 \mathrm{~cm}$ leg length rhombic gain with a $100 \Omega$ terminating resistance 


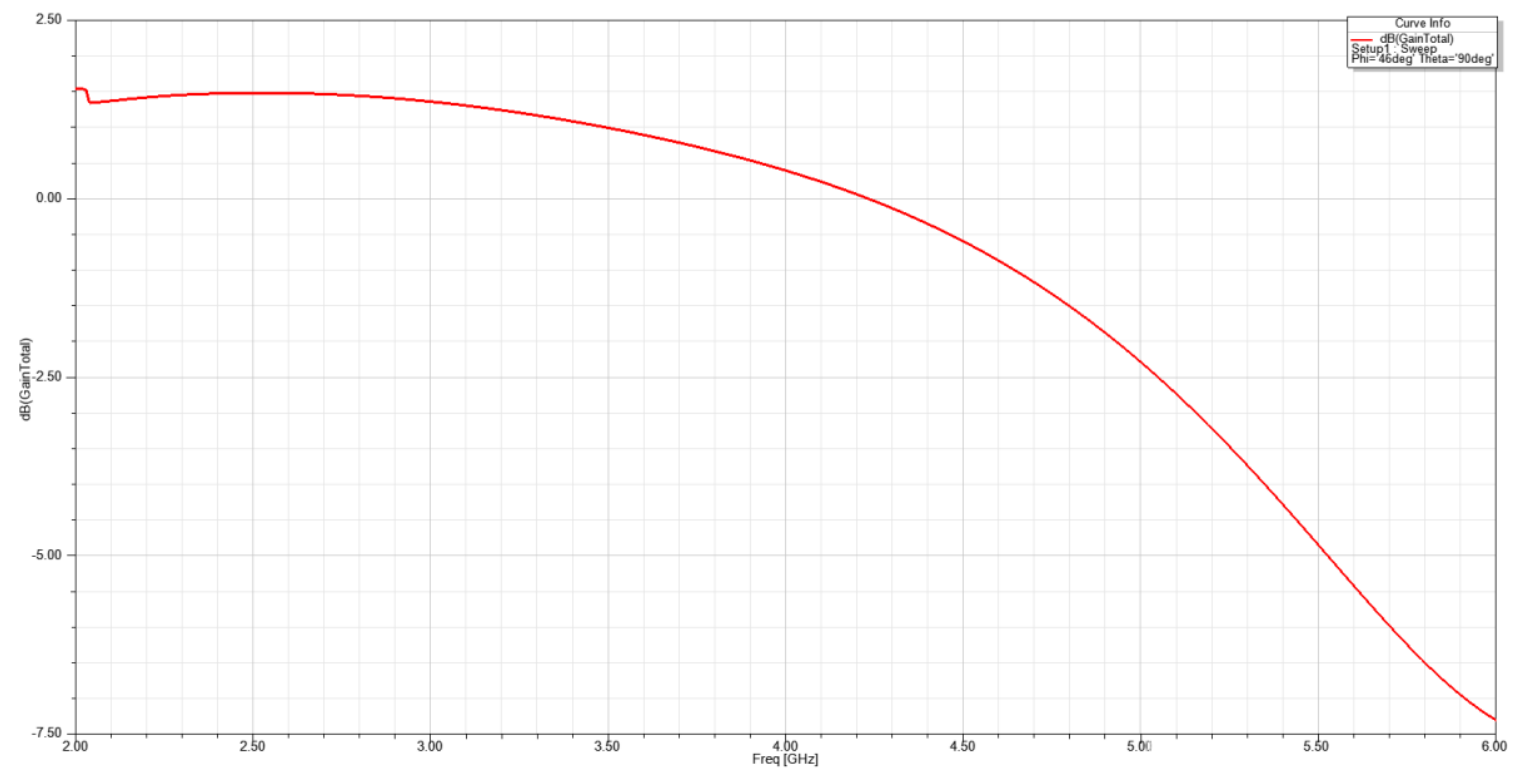

Figure 35: $2.5 \mathrm{~cm}$ leg length rhombic gain with a $100 \Omega$ terminating resistance

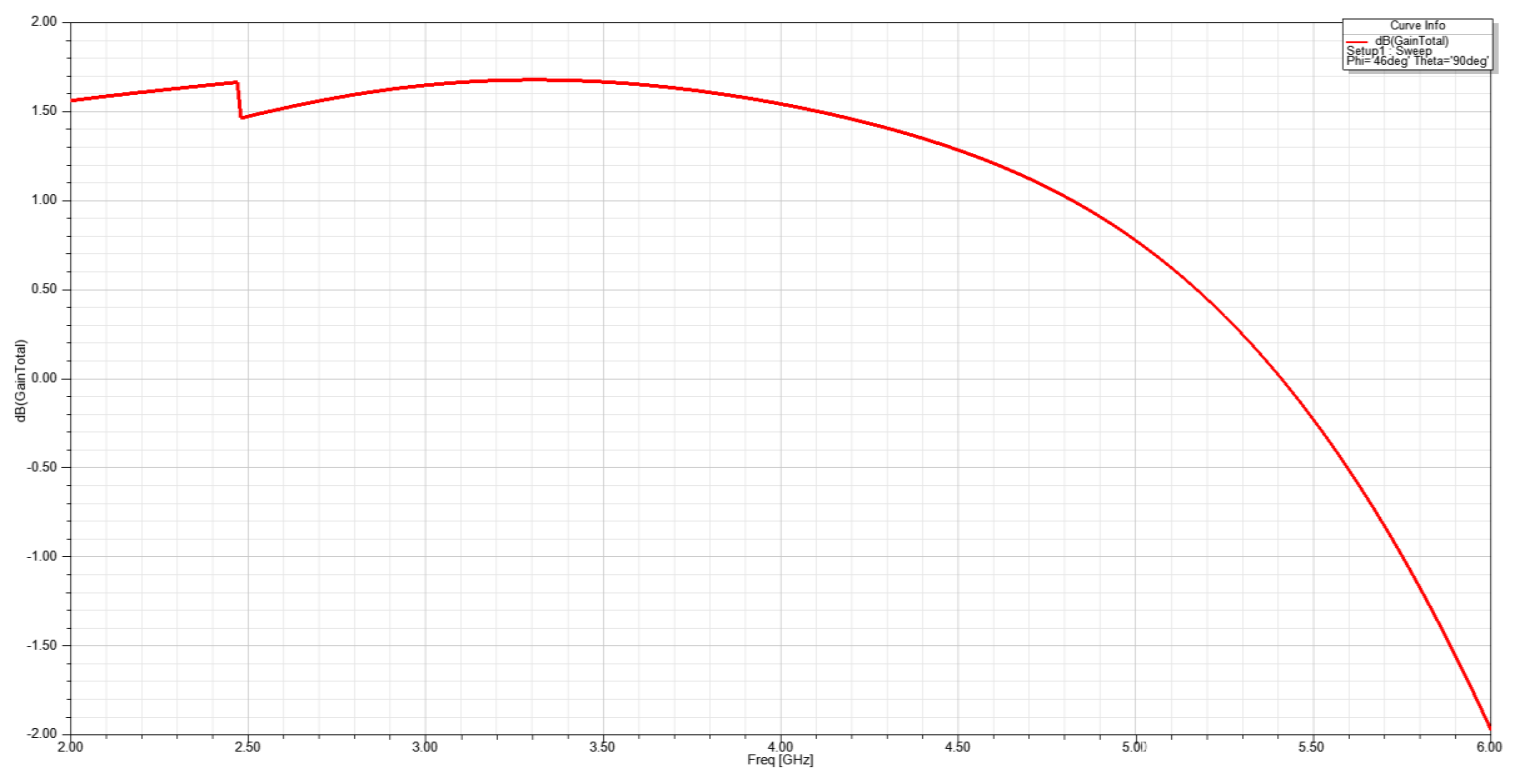

Figure 36: $2 \mathrm{~cm}$ leg length rhombic gain with a $100 \Omega$ terminating resistance

In the upper band rhombic simulations, the gain is a fair bit higher at $2.3 \mathrm{~cm}$ than it is when the rhombic leg length is increased to $2.5 \mathrm{~cm}$. When the leg length is shortened to 2 $\mathrm{cm}$, the gain at the higher frequencies improved significantly ( $3 \mathrm{~dB}$ at $6 \mathrm{GHz}$ ). The radiation pattern plots in Appendix A show that the $2 \mathrm{~cm}$ leg length plots are similar to the $2.3 \mathrm{~cm}$ plots. For that reason, a leg length of $2 \mathrm{~cm}$ and a terminating resistance of 100 
$\Omega$ will be used for the tests in chapter 5 for the upper band rhombic. The final radiation pattern plots for the lower band rhombic can be found in Appendix B.

\subsection{DF Simulations with HFSS Data}

After the HFSS simulations were complete, the focus shifted to the amplitude comparison monopulse DF simulations in MATLAB. These simulations were created using MATLAB's phased array toolbox. This allowed radiation pattern plots created in the HFSS simulations to be imported to MATLAB for use in DF simulations. As described in Chapter 2, the DF scenario consists of two rhombic antennas which have a 45 degree offset. The purpose of these DF simulations is to give the reader an idea of what parameters affect the target bearing error in an amplitude comparison monopulse implementation.

Once the data was imported, sources of error were introduced. Noise was introduced to the 2 channel amplitude monopulse DF system using MATLAB's awgn (Add White Gaussian Noise) function. Another error caused by the aircraft turning exists, but was not introduced into the simulation. This turning error was approximately $+/-0.1$ degrees per turn. This error is ignored since it should average out to zero over multiple turns. Path loss was also introduced to account for the signal loss through the air up to the height of the UAV.

Multiple scenarios were created to get a good idea of the DF accuracy. The changing parameters in these scenarios include the height of the UAV, SNR, and operating frequency. However, the goal of these simulations is to determine the effect of SNR and the height of the UAV on amplitude comparison monopulse by importing HFSS simulation data at a given frequency and rhombic leg length. 
With this in mind, the DF simulations for HFSS data were done for a $12.8 \mathrm{~cm}$ leg length at $800 \mathrm{MHz}$. The first simulation which was used as a baseline used the HFSS data for the $800 \mathrm{MHz} 12.8 \mathrm{~cm}$ leg length case with no noise or path loss. The error signal from performing amplitude comparison monopulse from this HFSS data is shown in Figure 37: Magnitude of monopulse error signal vs phi with no noise or path loss:

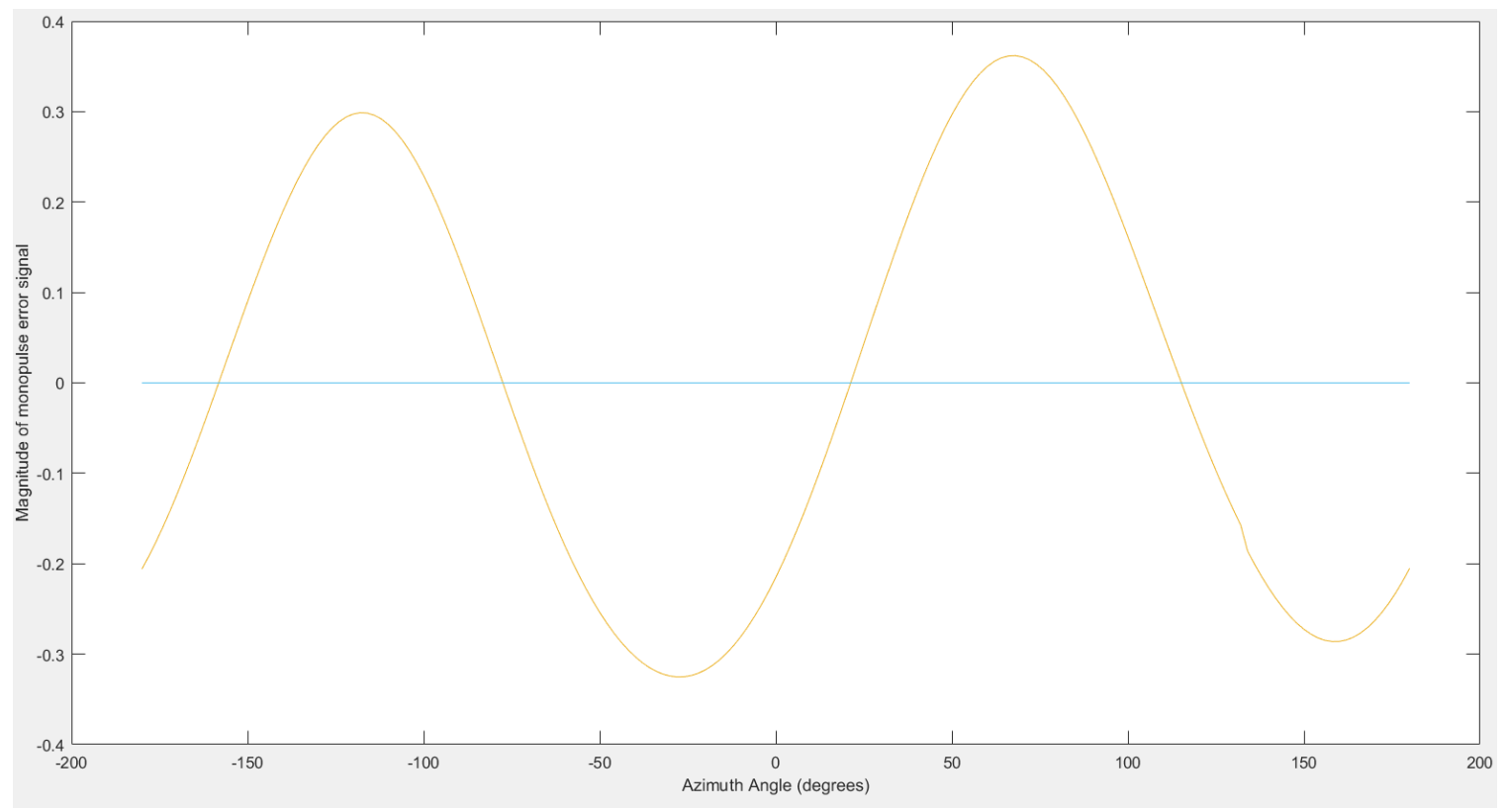

Figure 37: Magnitude of monopulse error signal vs phi with no noise or path loss

From the radiation patterns generated by the HFSS simulations earlier, it is known that the transmission source is around 45 degrees. This means that the transmitter or target would be around 20-25 degrees using amplitude comparison monopulse. The reason for this is that the 45 degree squint angle between the two sets of data from HFSS should have a HPBW intersection around 20-25 degrees. It is worth noting that HFSS only tracks data every 2 degrees, otherwise the HPBW intersection would be a more predictable value (likely close to 22.5 degrees). A zoomed in version of Figure 37: Magnitude of monopulse error signal vs phi with no noise or path loss is shown below: 


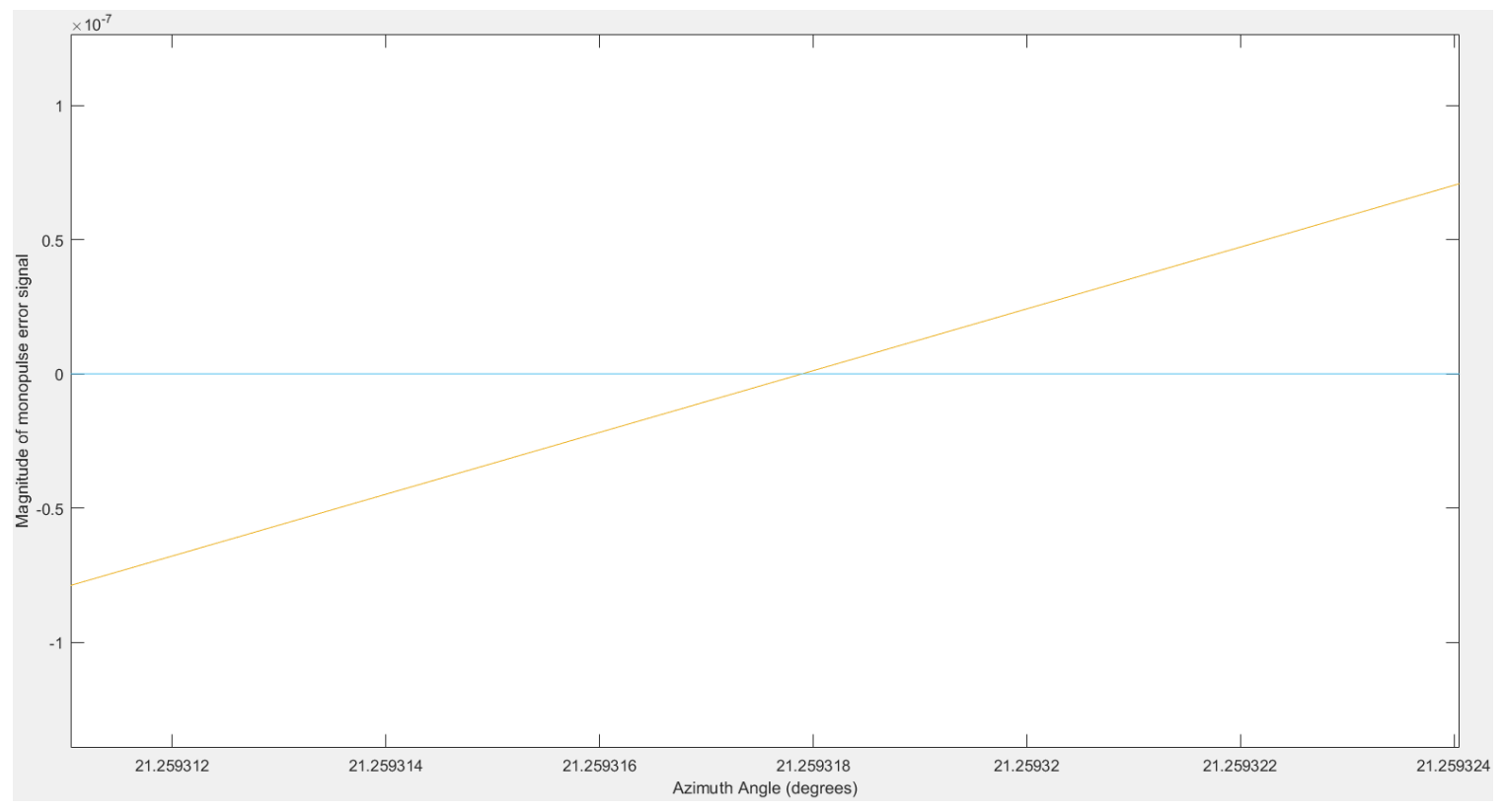

Figure 38: Zoomed magnitude of monopulse error signal vs phi with no noise or path loss From Figure 38: Zoomed magnitude of monopulse error signal vs phi with no noise or path loss, it can be seen that the error signal from HFSS data at $800 \mathrm{MHz}$ with a leg length of $12.8 \mathrm{~cm}$ that the transmission source is around 21.2593179 degrees. Tables showing cases of various SNR and UAV height values is shown below:

Table 2: Effect of SNR on monopulse error signal at $800 \mathrm{MHz}$ for a $12.8 \mathrm{~cm}$ leg length rhombic

\begin{tabular}{|c|c|}
\hline SNR $(\mathrm{dB})$ & Angle to HFSS transmission source $\left(^{\circ}\right)$ \\
\hline 0 & 17.496031 \\
\hline 5 & 18.443604 \\
\hline 10 & 19.278015 \\
\hline 15 & 19.933856 \\
\hline 20 & 19.641025 \\
\hline 25 & 20.432276 \\
\hline 30 & 21.188 \\
\hline 35 & 21.3138 \\
\hline 40 & 21.277468 \\
\hline
\end{tabular}

This table shows that the SNR of a system does not greatly affect the accuracy of amplitude comparison monopulse when it is above $30 \mathrm{~dB}$. This agrees with Figure 6: 
RMS angular error vs target angle-off-boresight for changing SNR [10] presented in the monopulse comparison section which had a similar accuracy change in response to changing SNR. This means that the SNR should not have an effect on the error of amplitude comparison monopulse of more than 1.5-2 degrees. Another table showing the change in HFSS transmission source angle relative to the change in UAV height is shown below:

Table 3: Effect of UAV height on monopulse error signal at $800 \mathrm{MHz}$ for a $12.8 \mathrm{~cm}$ leg length rhombic

\begin{tabular}{|c|c|}
\hline Height $(\mathrm{m})$ & Angle to HFSS transmission source $\left(^{\circ}\right)$ \\
\hline 500 & 21.259318 \\
\hline 1000 & 21.259318 \\
\hline 1500 & 21.259318 \\
\hline 2000 & 21.259318 \\
\hline 2500 & 21.259318 \\
\hline 3000 & 21.259318 \\
\hline 3500 & 21.259318 \\
\hline
\end{tabular}

There is no change in accuracy for amplitude comparison monopulse based on the height of the UAV. This makes sense since the changes based on path loss are the same at every point in phi assuming a stationary UAV when the difference in the azimuth patterns at different elevations are not changing. The MATLAB code which generated these scenarios is given in the Appendix C. 


\section{5 - Test Setup and Results}

\subsection{Test Setup}

The antenna testing is done using Carleton University's anechoic chamber. The rhombic antennas were tested so that the simulation results from the previous section could be verified. This is important since rhombic antennas had not been used at frequencies as high as this thesis' application requires. The anechoic chamber is enclosed by a Faraday Cage and has a turntable which the rhombic AUT (Antenna Under Test) is mounted on. The turntable turns 360 degrees allowing measurements to be taken 360 degrees in the azimuth direction. It also has a controller which is connected to a computer in the lab adjacent to the anechoic chamber. The computer controls both the turntable and a spectrum analyzer which is connected to the AUT. The test setup is shown in the following two figures: 


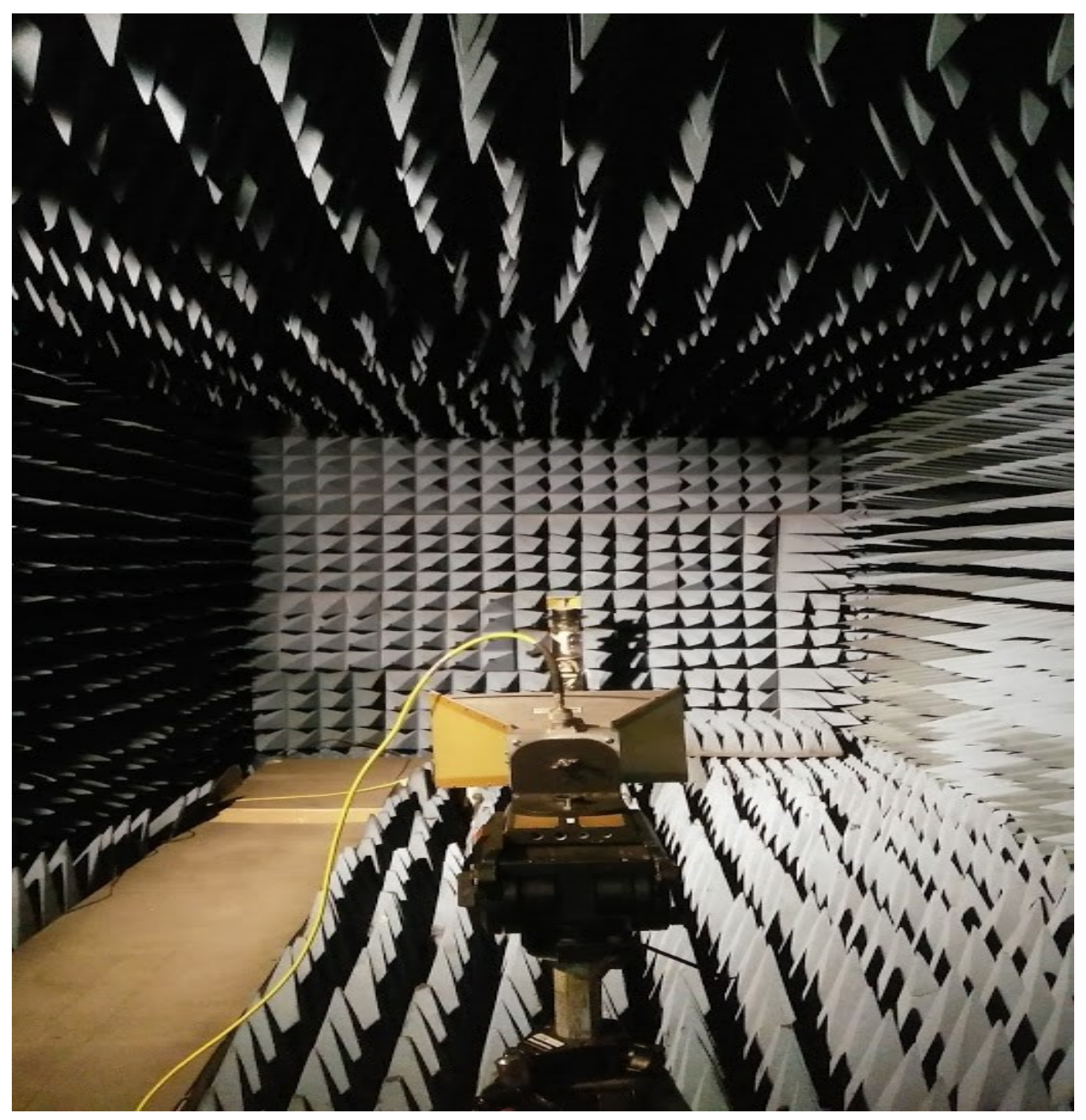

Figure 39: Anechoic chamber with transmit and receive antennas 


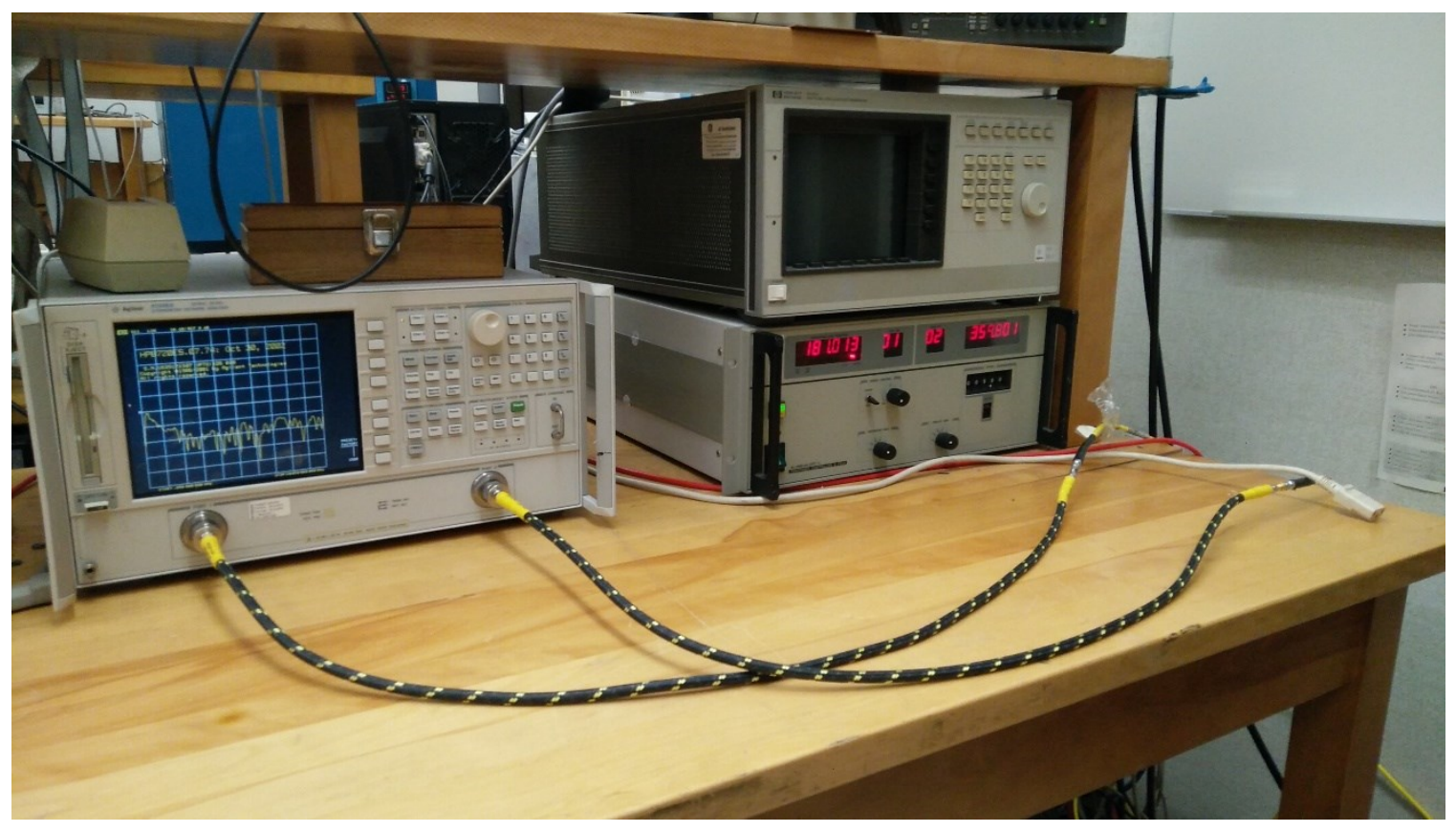

Figure 40: Carleton University's anechoic chamber lab. The spectrum analyzer is on the left and the turntable controller is on the bottom right

This thesis could be applicable at frequencies much lower than $600 \mathrm{MHz}$, however the anechoic chamber at Carleton University is not capable of taking measurements at frequencies lower than $600 \mathrm{MHz}$. Measurements can't be performed at those frequencies because the chamber is too small and the gain standards only go down to $600 \mathrm{MHz}$. Gain results were done from 2-6 GHz due to a lack of a suitable antenna in the $0.6-1.4 \mathrm{GHz}$ range at Carleton University’s anechoic chamber lab.

For each antenna design from the design/simulation section, the S11 parameter is measured. This is done using the spectrum analyzer in Carleton University's anechoic chamber lab. Next, the $0.6-6 \mathrm{GHz}$ gain standard is mounted on the turntable at the back of the anechoic chamber shown in the figure above. Once the gain standard is measured, the AUT is mounted on the turntable and the AUT gain measurements were performed. The results of this procedure are shown in the following section. In addition to these 
parameters, the S21 is measured for each rhombic. Note that all measurements are done in the azimuth plane (elevation angle of $90^{\circ}$ ).

Figure 41: $6 \mathrm{~cm}$ leg length rhombic antenna showing the first lower band rhombic antenna that was tested is shown below.

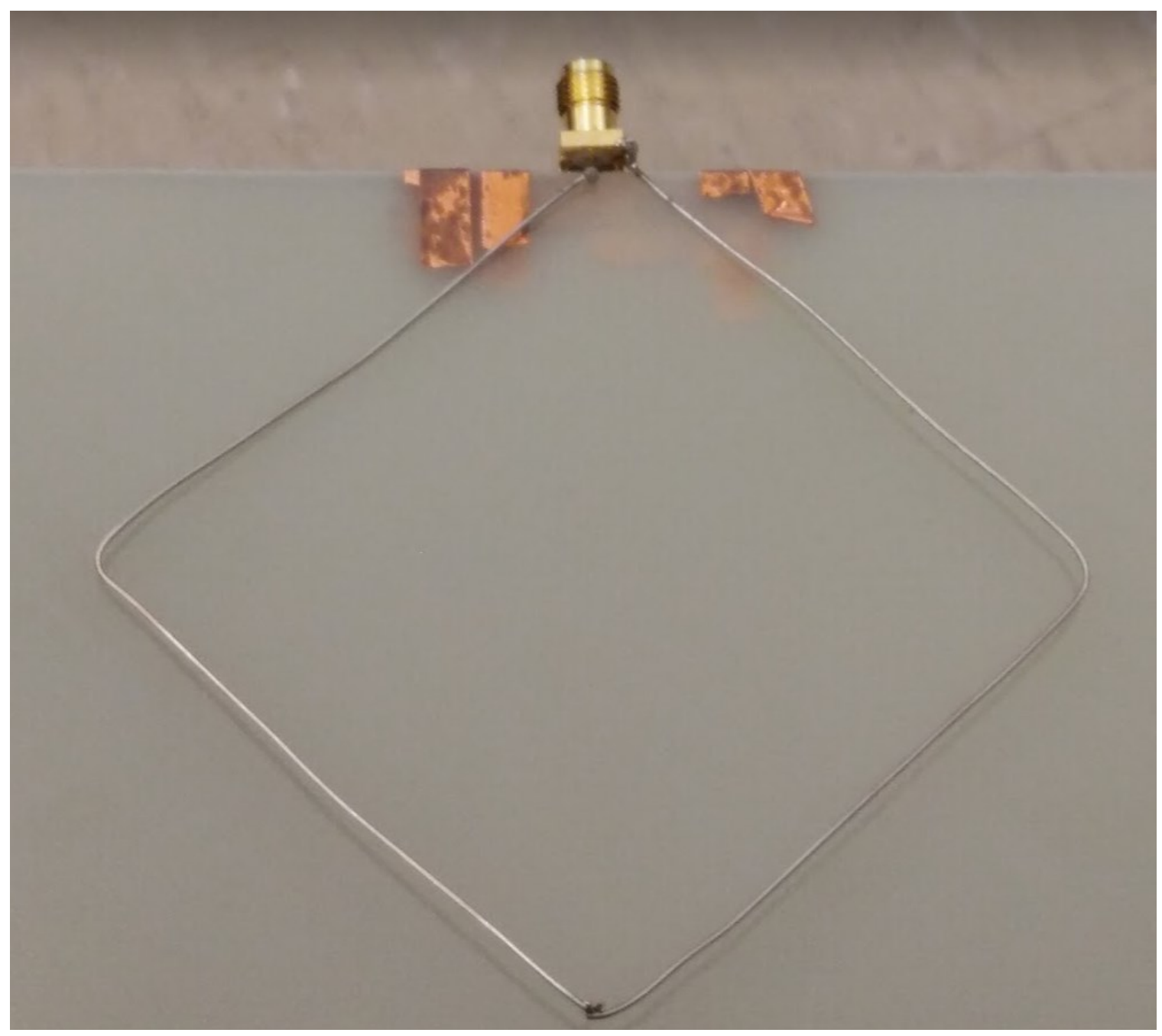

Figure 41: $6 \mathrm{~cm}$ leg length rhombic antenna

\subsection{Initial Test Results}

\subsubsection{Upper Band Test Results}

The first tests were done on the upper band rhombic. The leg length of the rhombic that is built for the 2-6 GHz frequency range is $2 \mathrm{~cm}$. Tests are performed for this leg length 
with a $100 \Omega$ terminating resistance. The following figures show the results of the anechoic chamber tests at the frequencies that were simulated in Chapter 4 . The first figure shows the S11 from 2-6 GHz.

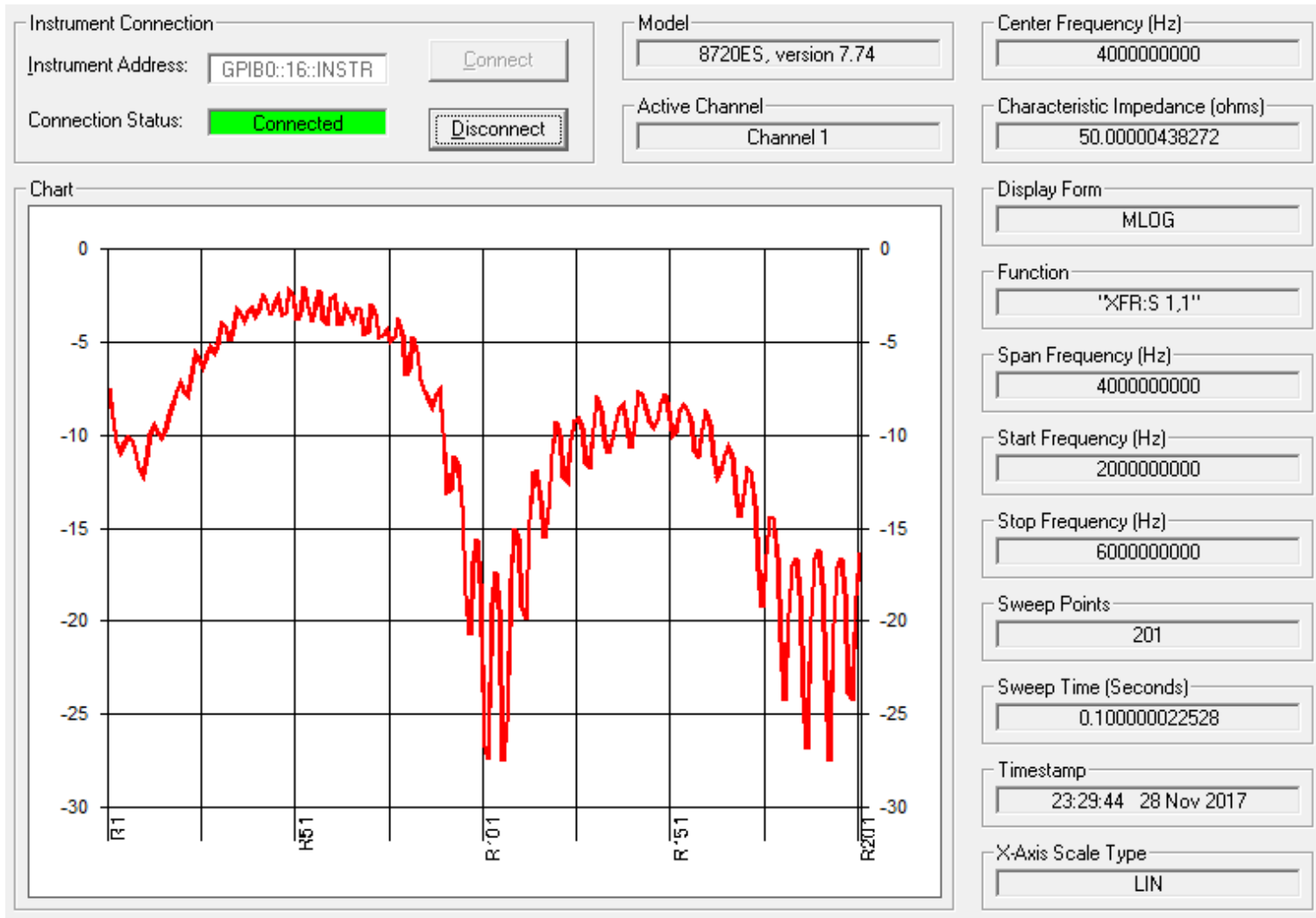

Figure 42: S11 of assembled upper band rhombic

The S11 plot above shows far better S11 performance than the simulated performance shown in Figure 31: S11 for $2.3 \mathrm{~cm}$ leg length rhombic with a $100 \Omega$ terminating resistance. The difference is likely due to the smaller rhombic leg length improving the S11 performance at the higher frequencies as well as an imperfect apex angle.

It is important to note that all of the following S21 measurements for the entirety of Chapter 5 are in $\mathrm{dB}$ and are measured on the azimuth plane. The azimuth direction from 
$0-360$ is marked on the outside of the plots and the $\mathrm{S} 21$ in $\mathrm{dB}$ is marked on the inner rings of the plots.

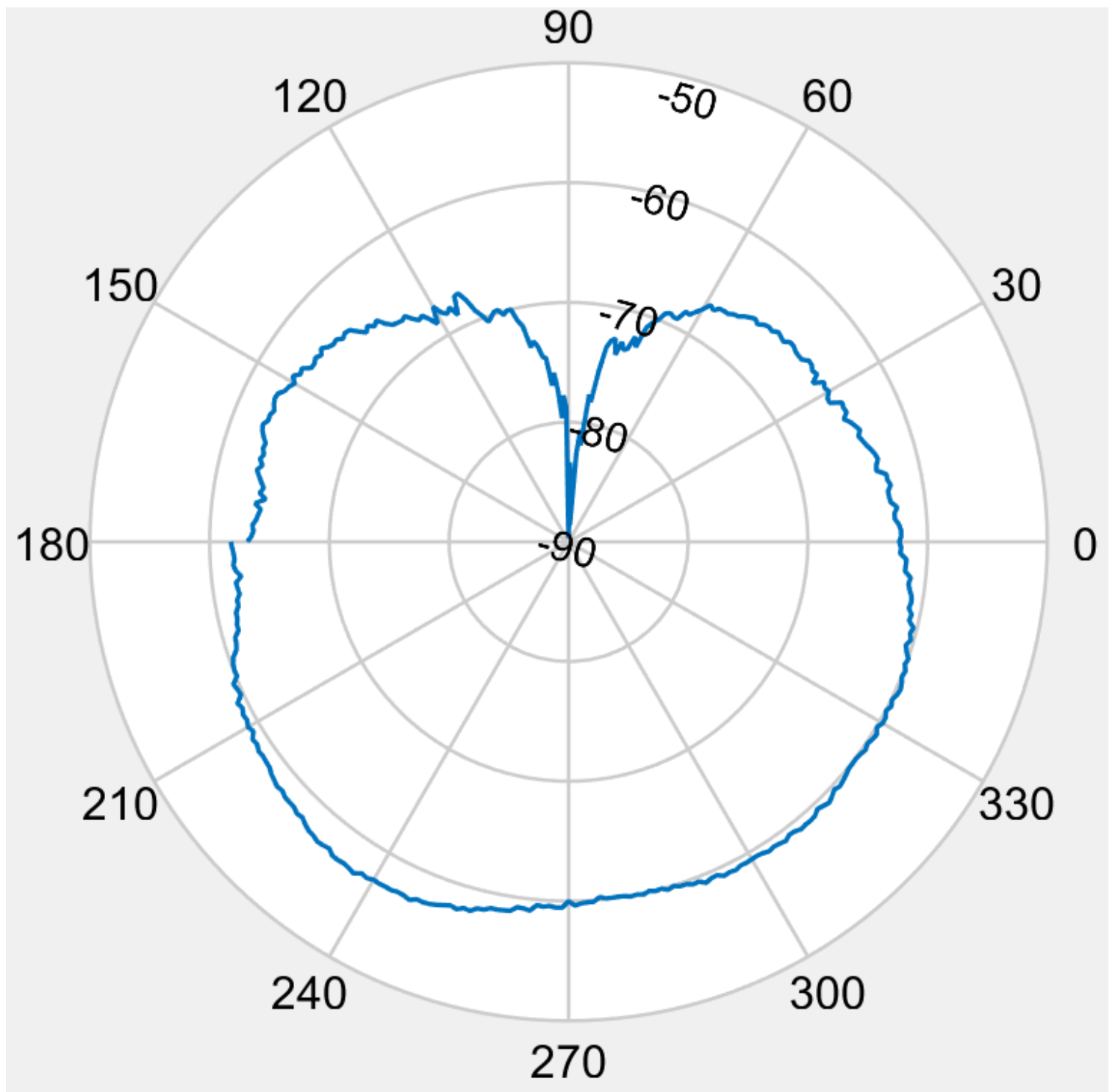

Figure 43: $2 \mathrm{GHz} S 21(d B)$ in azimuth plane for $2 \mathrm{~cm}$ leg length rhombic 


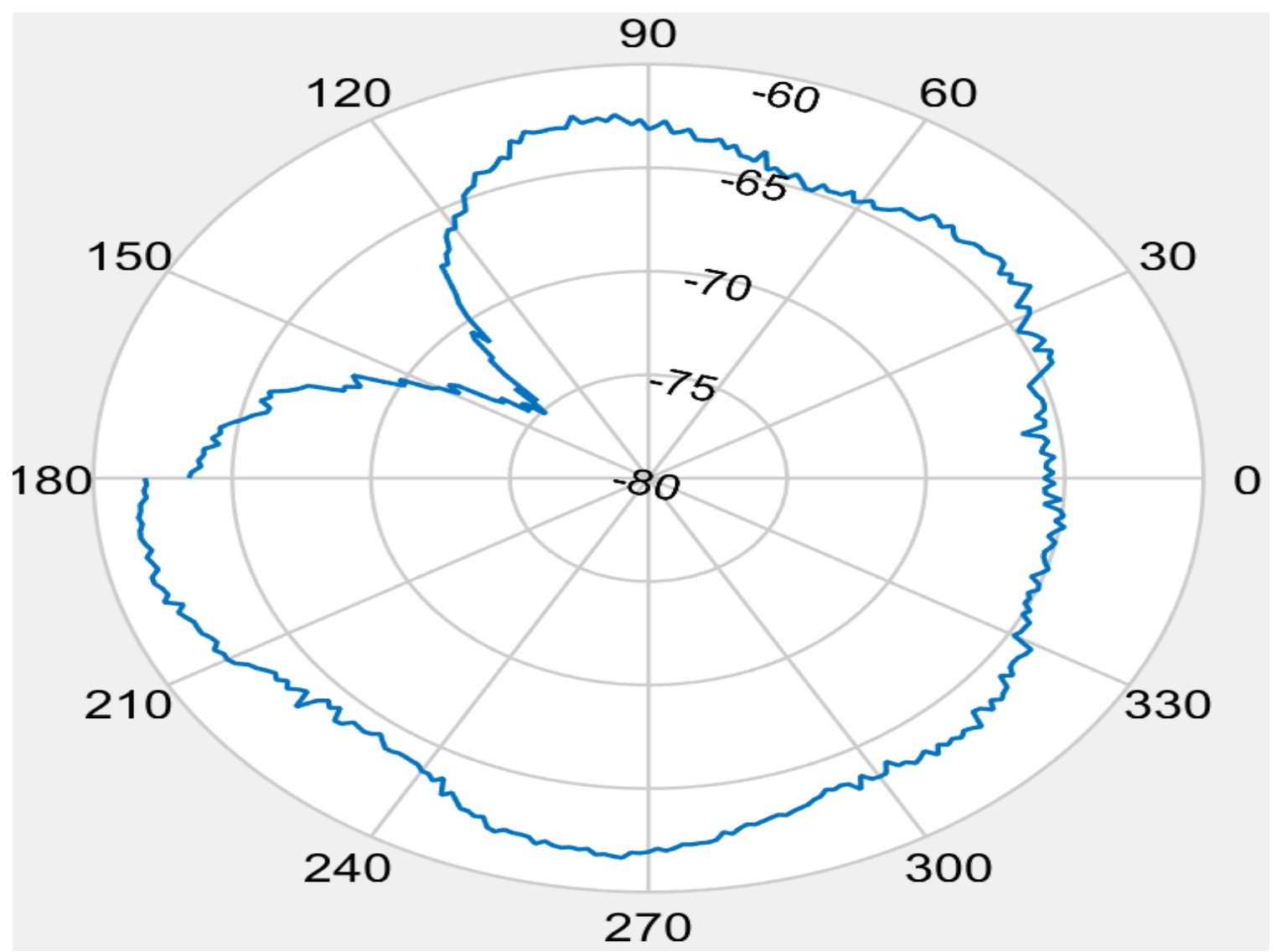

Figure 44: $2.5 \mathrm{GHz} S 21(d B)$ in azimuth plane for $2 \mathrm{~cm}$ leg length rhombic

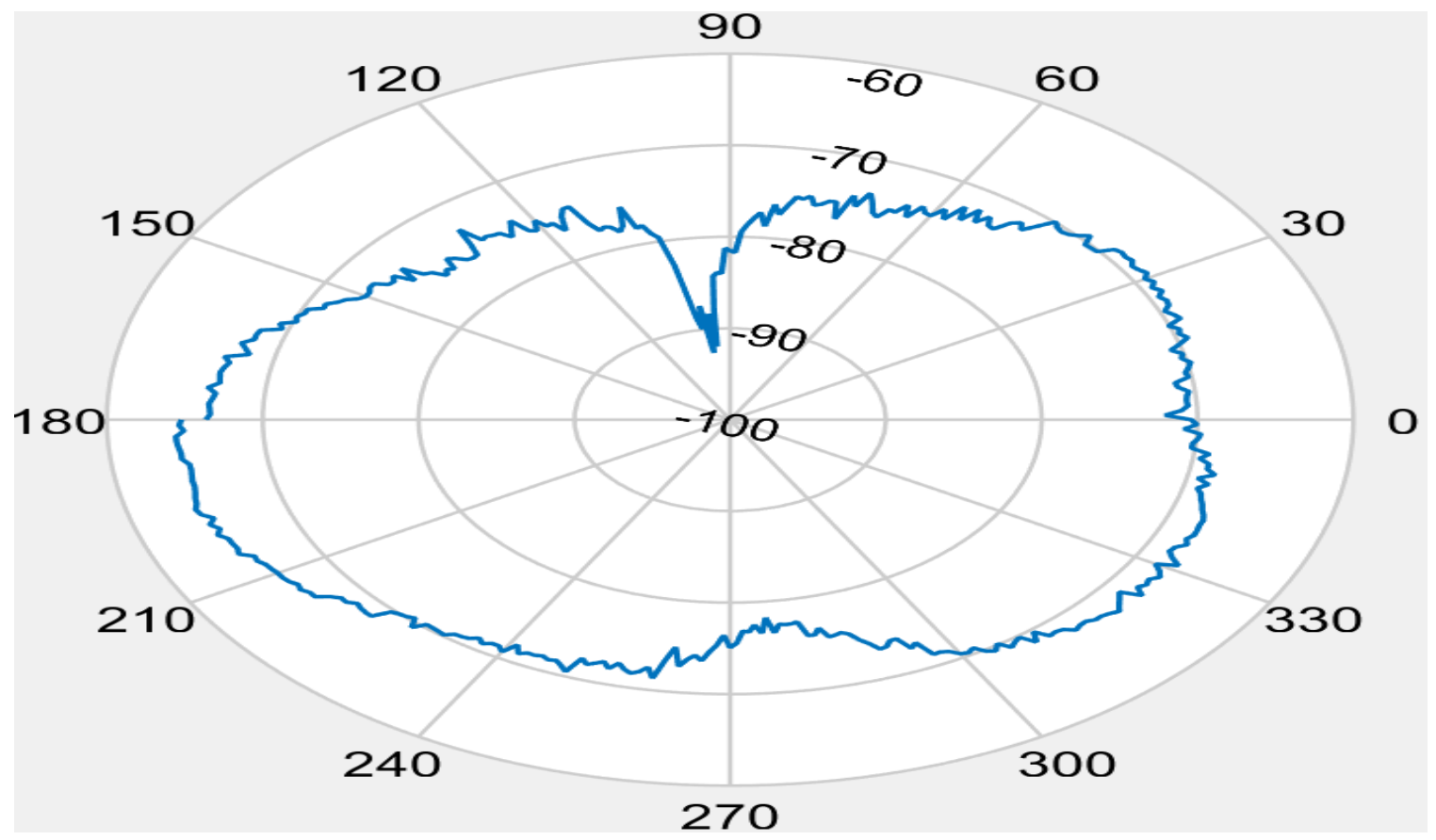

Figure 45: $3 \mathrm{GHz} S 21(d B)$ in azimuth plane for $2 \mathrm{~cm}$ leg length rhombic 


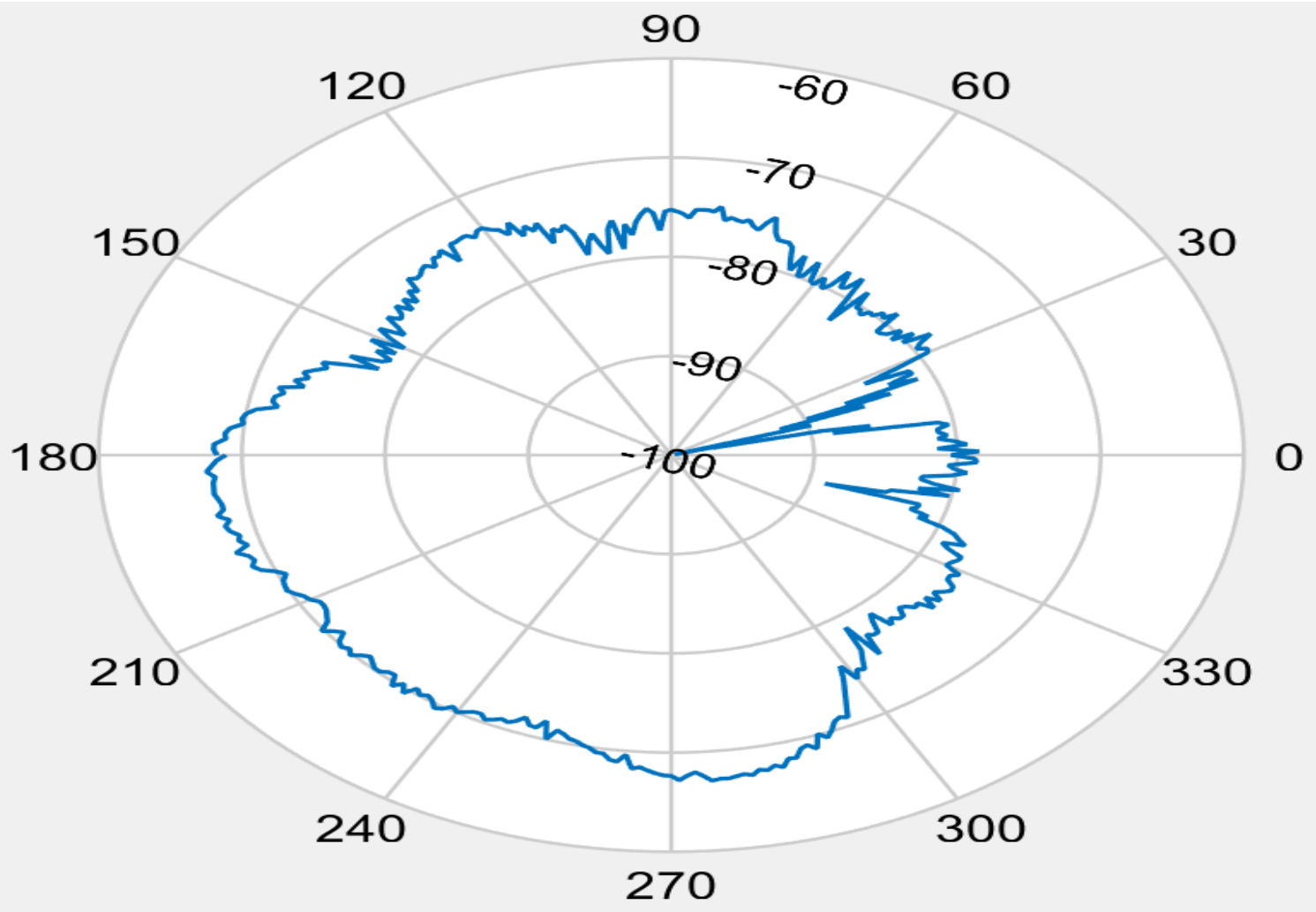

Figure 46: $3.5 \mathrm{GHz} S 21(d B)$ in azimuth plane for $2 \mathrm{~cm}$ leg length rhombic

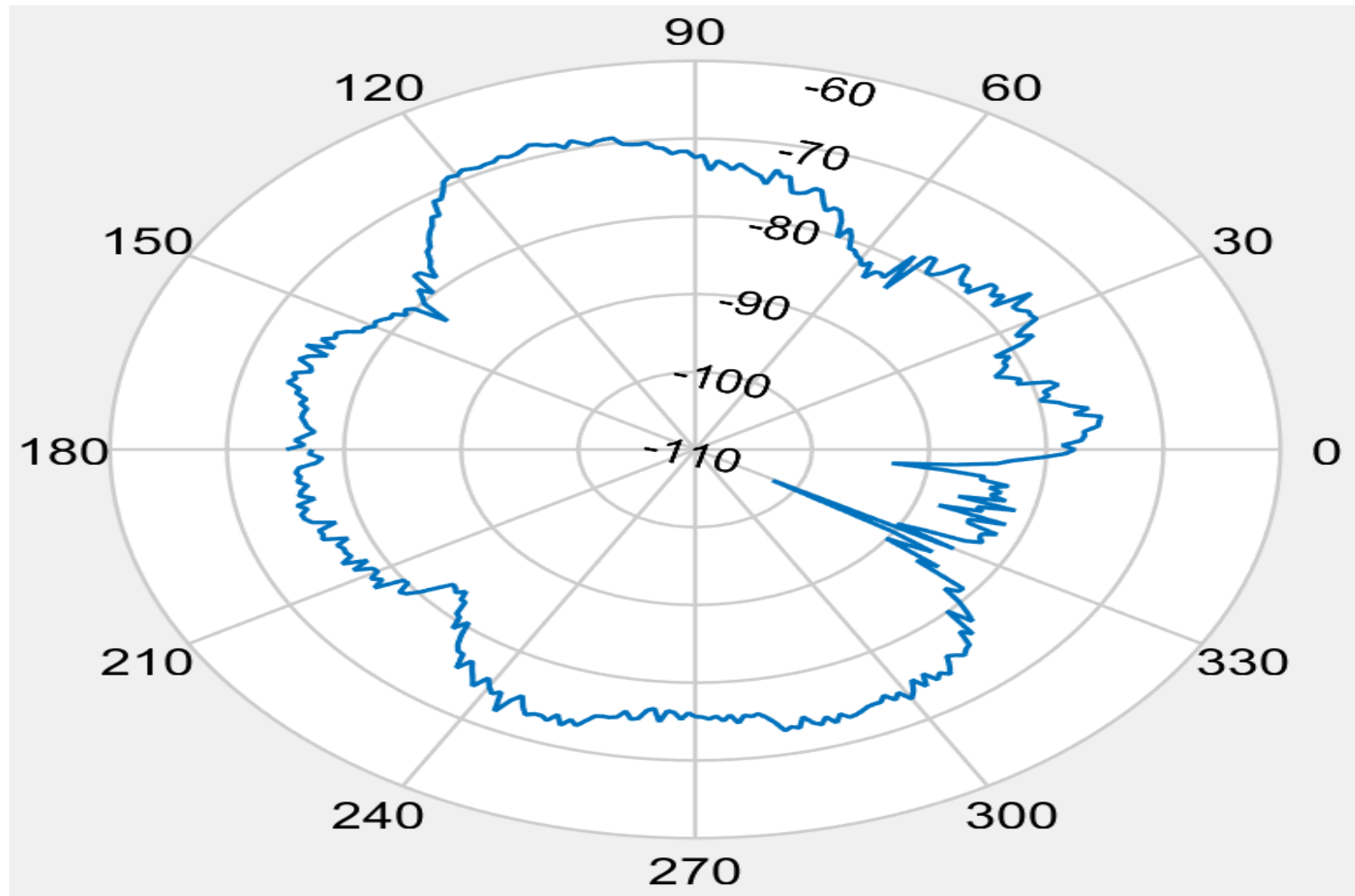

Figure 47: $4 \mathrm{GHz} S 21(d B)$ in azimuth plane for $2 \mathrm{~cm}$ leg length rhombic 


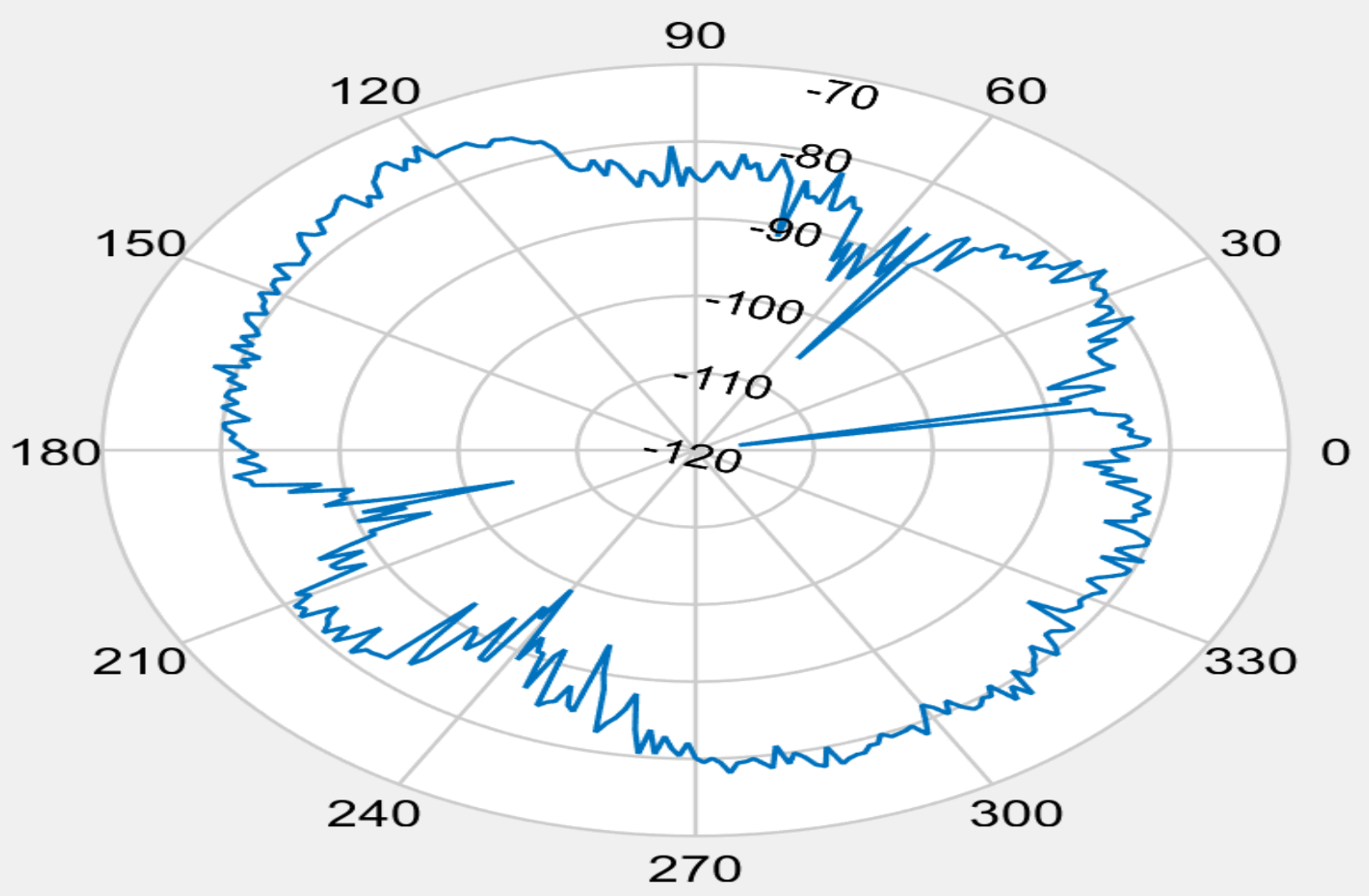

Figure 48: 4.5 GHz S21 $(d B)$ in azimuth plane for $2 \mathrm{~cm}$ leg length rhombic

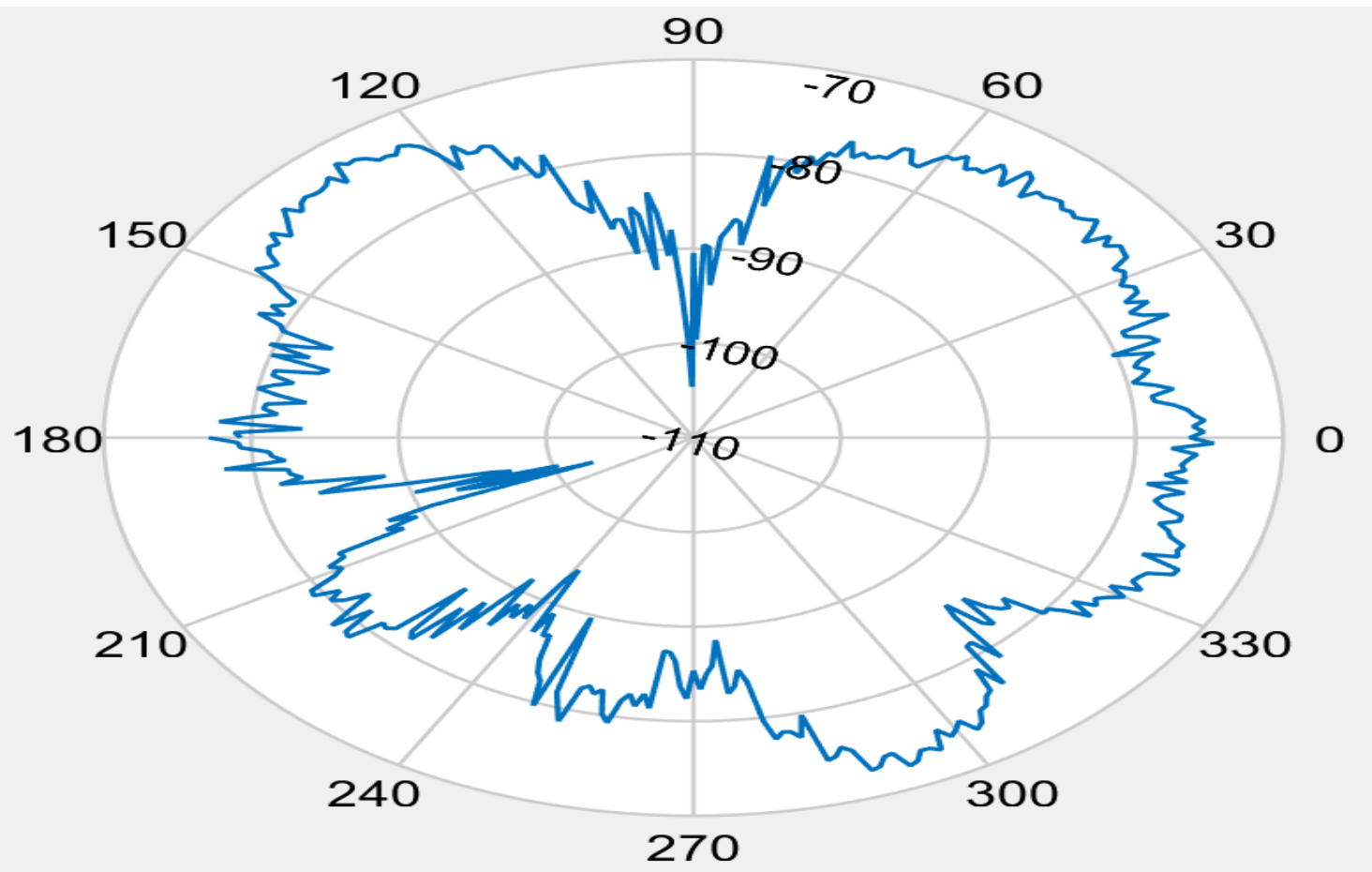

Figure 49: $5 \mathrm{GHz} S 21(d B)$ in azimuth plane for $2 \mathrm{~cm}$ leg length rhombic 


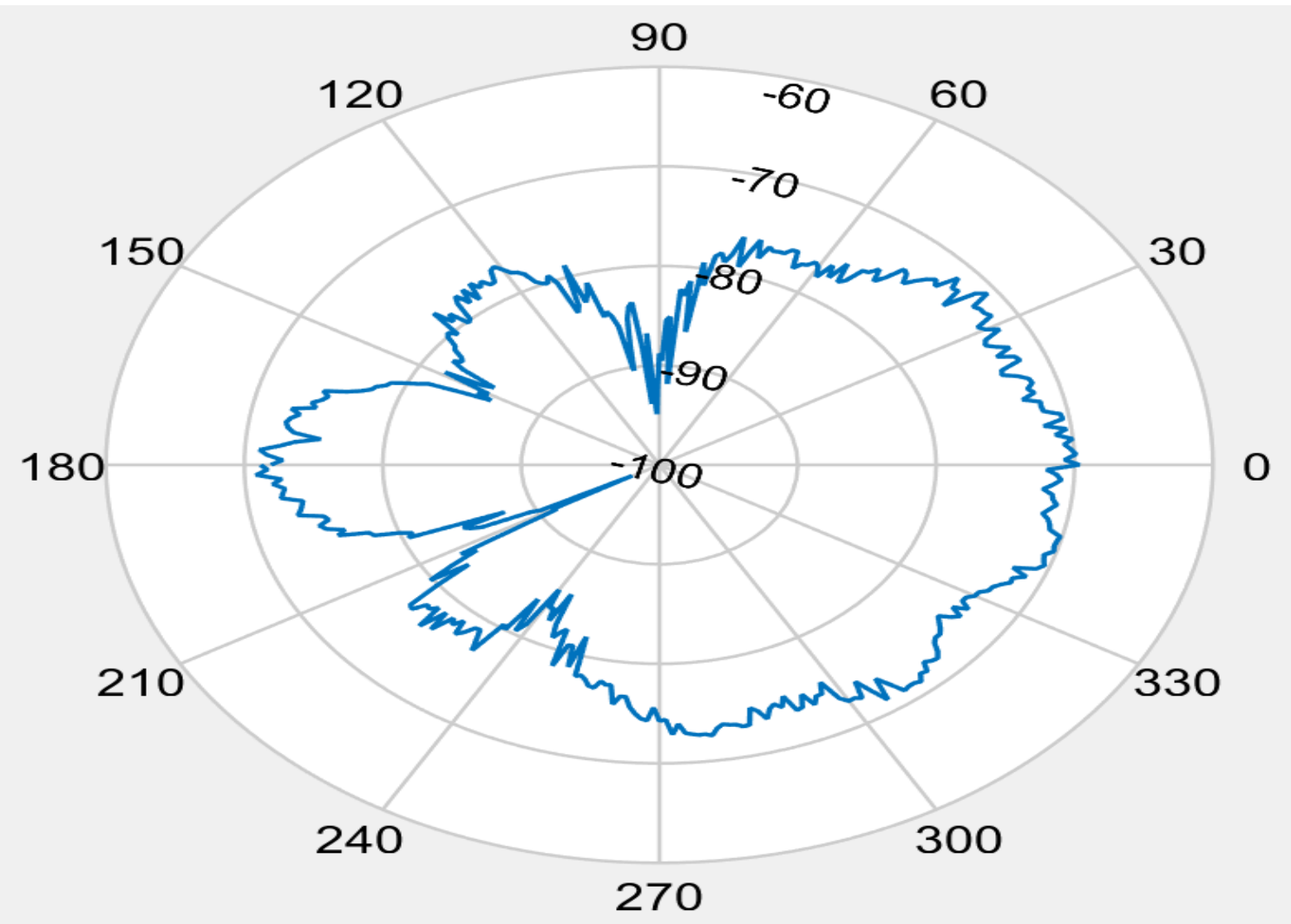

Figure 50: $5.5 \mathrm{GHz} S 21(d B)$ in azimuth plane for $2 \mathrm{~cm}$ leg length rhombic

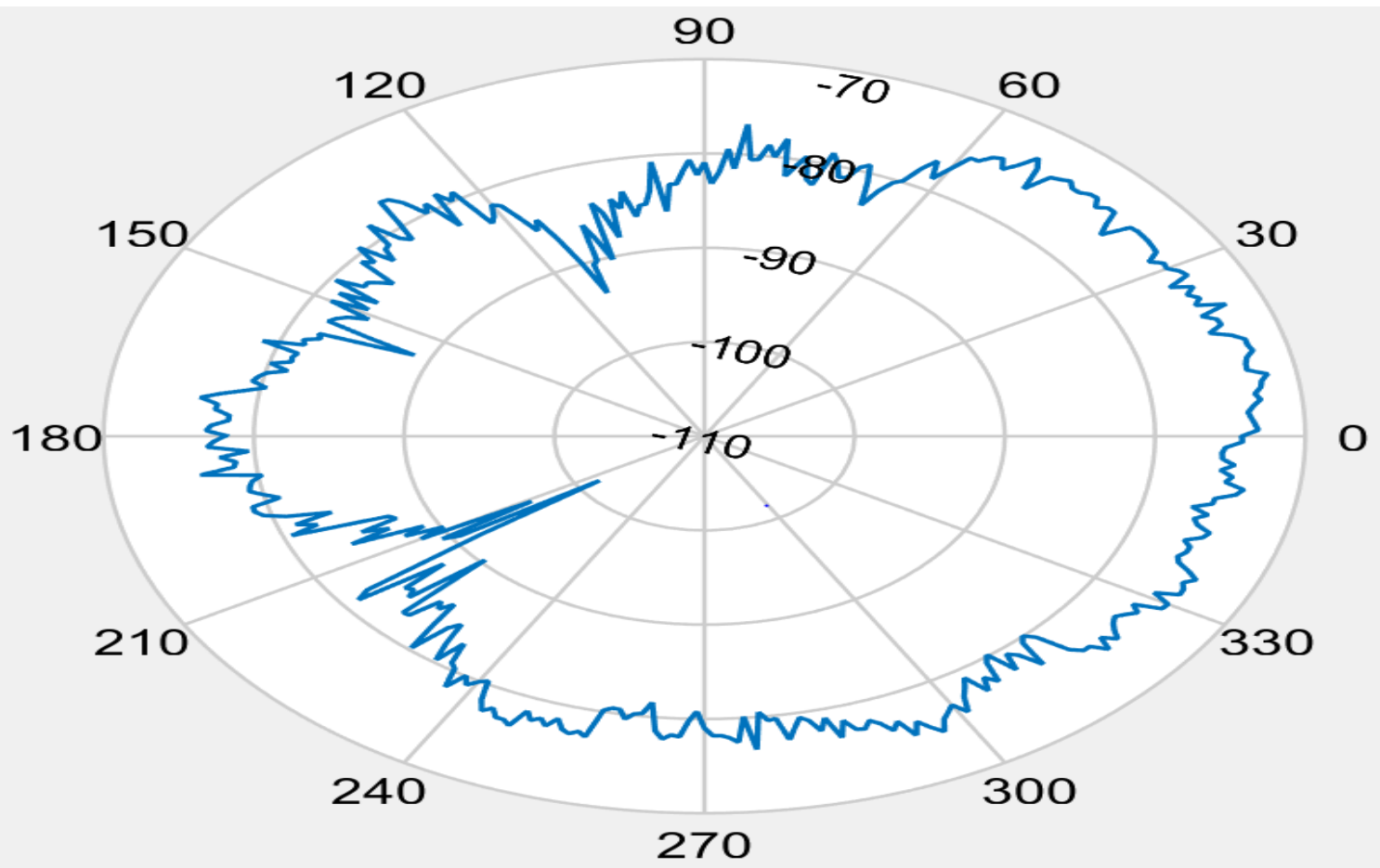

Figure 51: $6 \mathrm{GHz} S 21(d B)$ in azimuth plane for $2 \mathrm{~cm}$ leg length rhombic 
It can be seen in the figures above that with an increasing frequency the noise in the S21 data is increasing. This makes sense since an imperfect terminating resistor, wire antenna, and spectrum analyzer are used for the measured results introducing high frequency noise components that are not found in simulations with perfect electric conductors. The HPBW of the measured results closely follows the simulated results in Appendix B with the exception of the 2 and $2.5 \mathrm{GHz}$ S21 measurements. This could be corrected by increasing the leg length of the rhombic slightly to improve the HPBW at these frequencies. This is left as future work since the upper band rhombic results will not be used for the proof of concept for the DF portion of this thesis.

\section{Gain Results}

The gain-transfer method was used to find the gain of the rhombics. This method involves using two antennas with a known gain and bandwidth and a third antenna with an unknown gain over the other antennas operating band.

The two known antennas used as standards were a BAE-H-1734 Double-Ridged Broadband Horn and a Broadband Electro-Metrics Horn (model EM-6961) which operate between $0.6-6 \mathrm{GHz}$ and $1.4-1.8 \mathrm{GHz}$ and $1.9-18 \mathrm{GHz}$ respectively. They have average power gains of $9 \mathrm{~dB}$ and $10.7 \mathrm{~dB}$ respectively. The BAE-H-1734 antenna was used as the transmitter, while the rhombics and the EM-6961 were used as receivers. The gain curve of the EM6961 from its datasheet is shown below: 


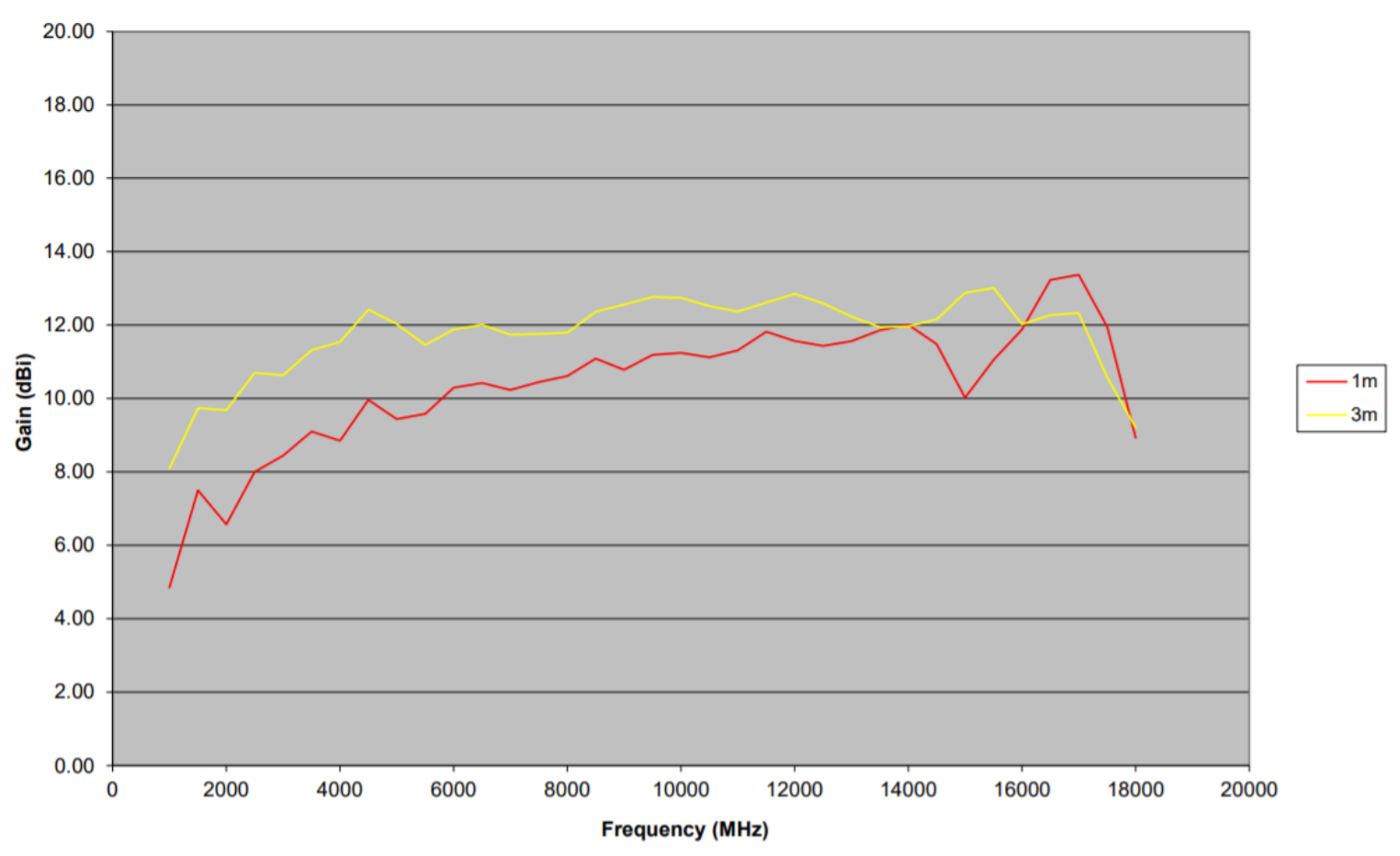

Figure 52: Gain of the EM6961 gain standard vs frequency [17]

Note that the version of the EM6961 that is used was the $1 \mathrm{~m}$ version. Therefore, gain calculations are done using the curve starting with a gain below $6 \mathrm{~dB}$ from the figure above.

The gain of the upper band rhombic is calculated on a point by point basis for each frequency that was measured using the following formula:

$$
G_{\text {AUT }}=G_{\text {standard }}+\left(P_{\text {AUT }}-P_{\text {standard }}\right)
$$

Where $\mathrm{G}_{\text {AUT }}$ is the gain of the AUT (the upper band rhombic in this case), $\mathrm{G}_{\text {standard }}$ is the gain of the EM6961 antenna, $\mathrm{P}_{\mathrm{AUT}}$ is the received power of the AUT, and $\mathrm{P}_{\text {standard }}$ is the received power of the EM6961 antenna. This formula can be used to calculate the maximum, minimum, and total gain of the upper band rhombic antenna. The maximum and minimum gains is calculated using the maximum power received by the EM6961 standard and maximum and minimum power received for the rhombic AUT respectively. 
A plot showing the total gain vs frequency from $2-6 \mathrm{GHz}$ is shown below. Note that total gain is calculated summing the maximum gain (calculated using the maximum $\mathrm{P}_{\text {AUT }}$ ) and minimum gain (calculated using the maximum $\mathrm{P}_{\mathrm{AUT}}$ ) of the rhombic AUT. The lower band antenna gain is not tested since the EM6961 gain standard can not cover the 0.6 $1.4 \mathrm{GHz}$ frequency range.

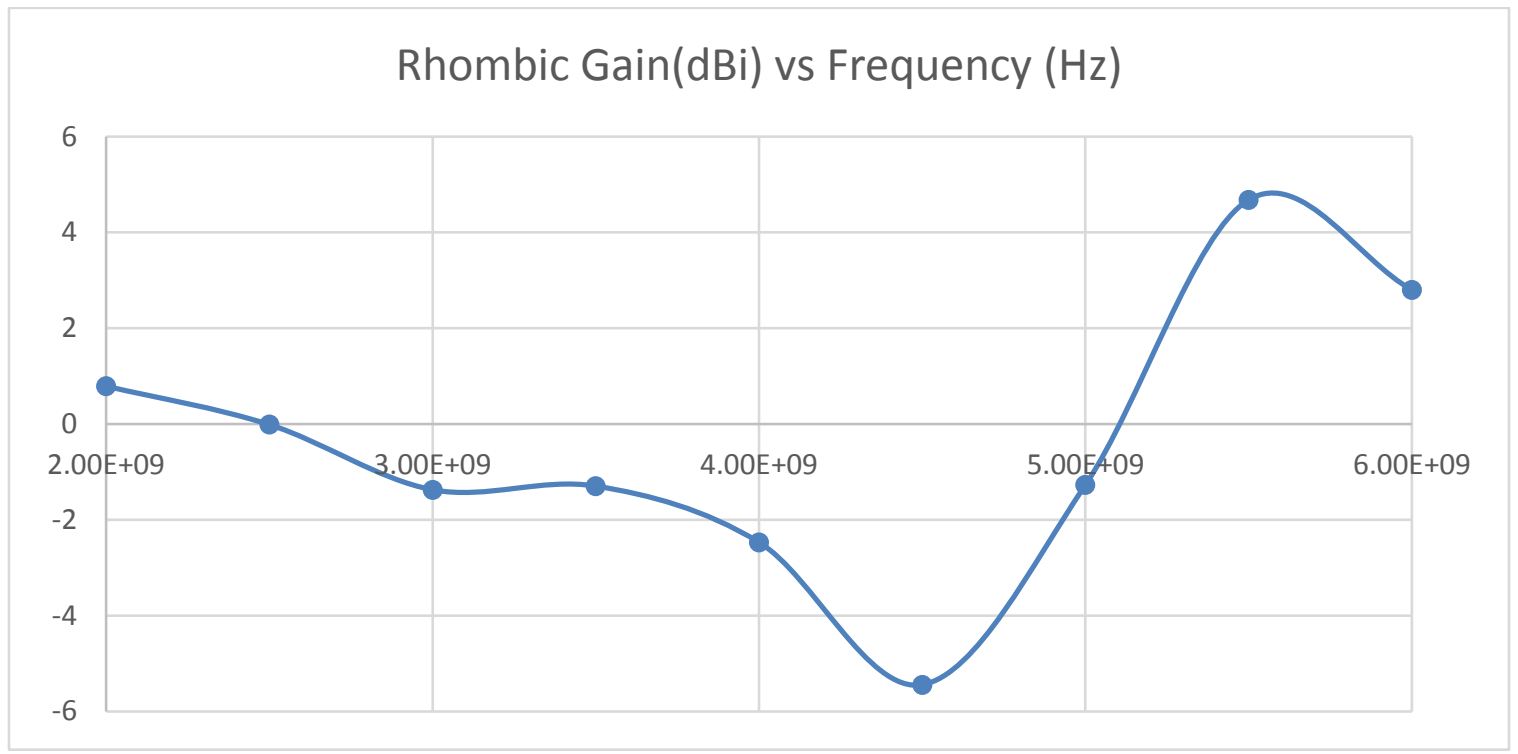

Figure 53: Total gain (dBi) vs frequency $(\mathrm{Hz})$

The total gain given by Figure 53: Total gain ( $\mathrm{dBi}$ ) vs frequency above has worse gain characteristics over most of the band when compared to the gain figure that was simulated in HFSS for a $2 \mathrm{~cm}$ leg length rhombic. This is likely caused by imperfections in the shape of the antenna and the increased terminating resistance.

Note that polarization loss factor does not need to be taken into account here since the antennas used in the chamber were horizontally polarized and rhombic antennas are also horizontally polarized [18]. 


\subsubsection{Lower Band Test Results}

The lower band rhombic is tested in the same manner as the upper band rhombic. It is a 6 $\mathrm{cm}$ leg length rhombic antenna with a $600 \Omega$ terminating resistance (620 $\Omega$ is used since it is the closest available to 600 ) operating from $0.6-2 \mathrm{GHz}$. S21 chamber results using a $6 \mathrm{~cm}$ leg length from $0.6-2 \mathrm{GHz}$ in steps of $200 \mathrm{MHz}$ are shown below. Preceding these results is an S11 plot for the assembled rhombic.
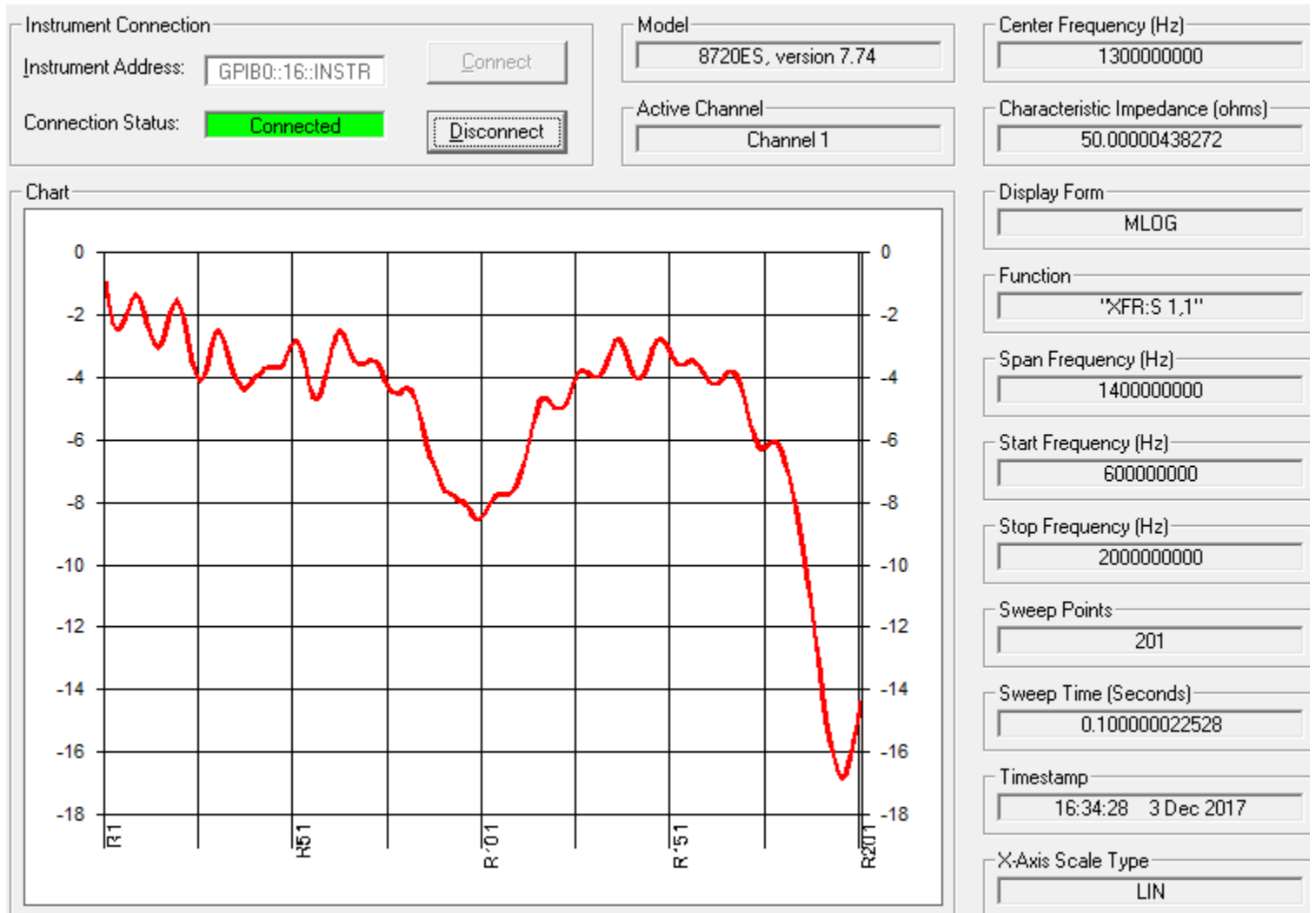

Figure 54: S11 for the lower band assembled rhombic

The S11 above is slightly better than the simulated S11 shown in Figure 32: S11 for $6 \mathrm{~cm}$ leg length rhombic with a $600 \Omega$ terminating resistance with a similar shape. The differences between the two figures are likely caused by the slightly larger imperfect resistor used in the assembled rhombic. 


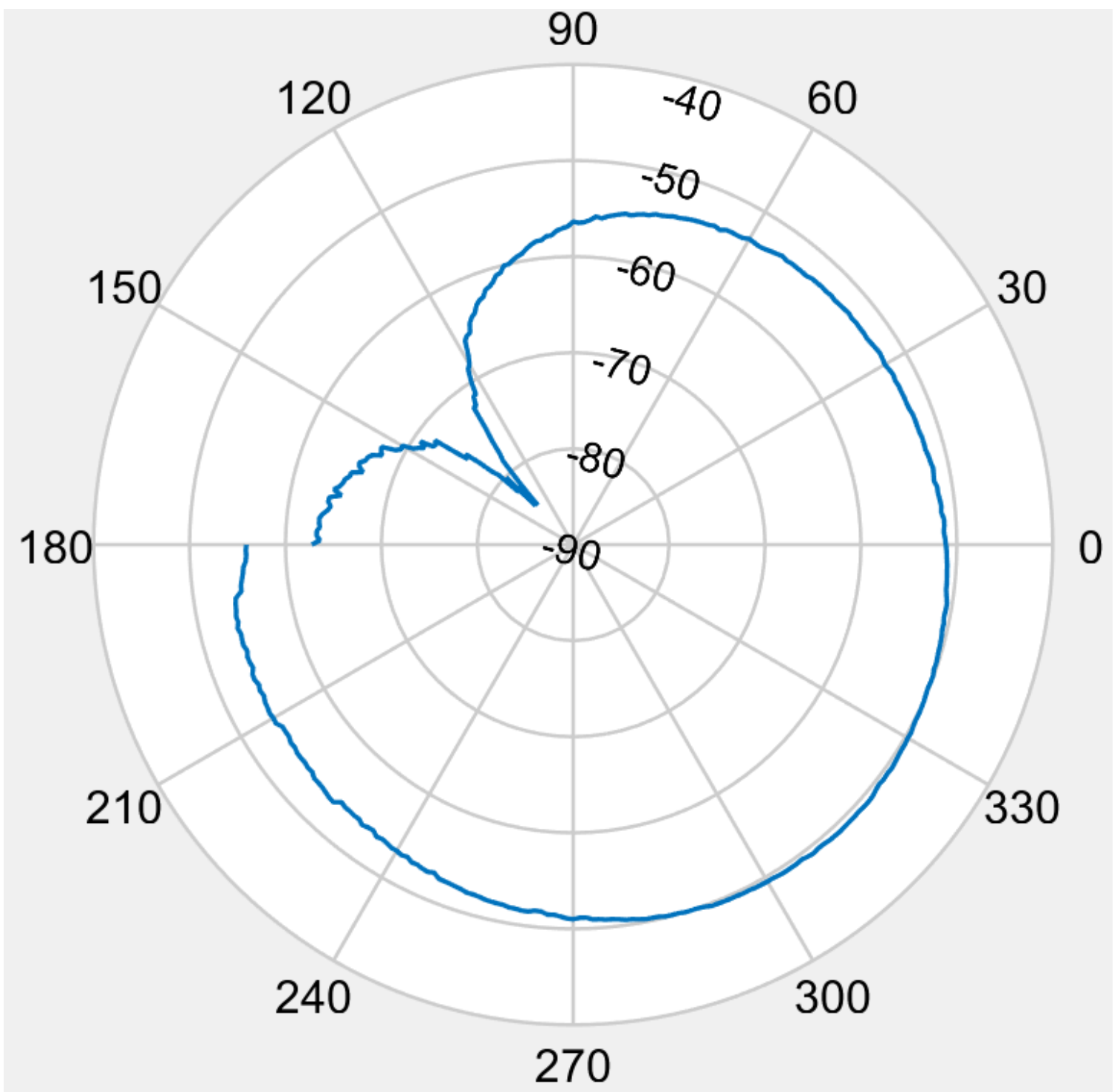

Figure 55: 0.6 GHz S21 $(d B)$ in azimuth plane for $6 \mathrm{~cm}$ leg length rhombic 


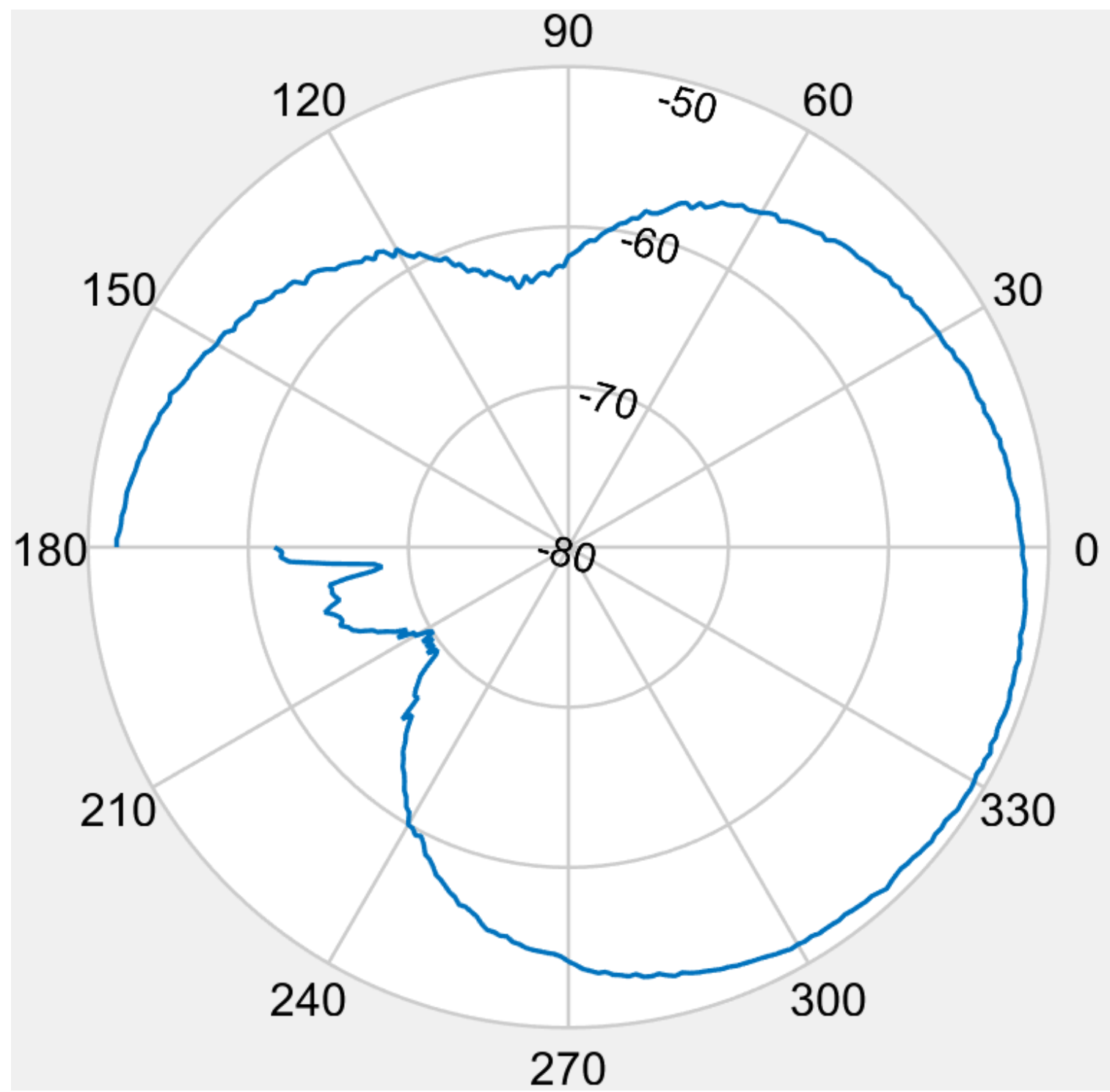

Figure 56: $0.8 \mathrm{GHz} S 21(d B)$ in azimuth plane for $6 \mathrm{~cm}$ leg length rhombic 


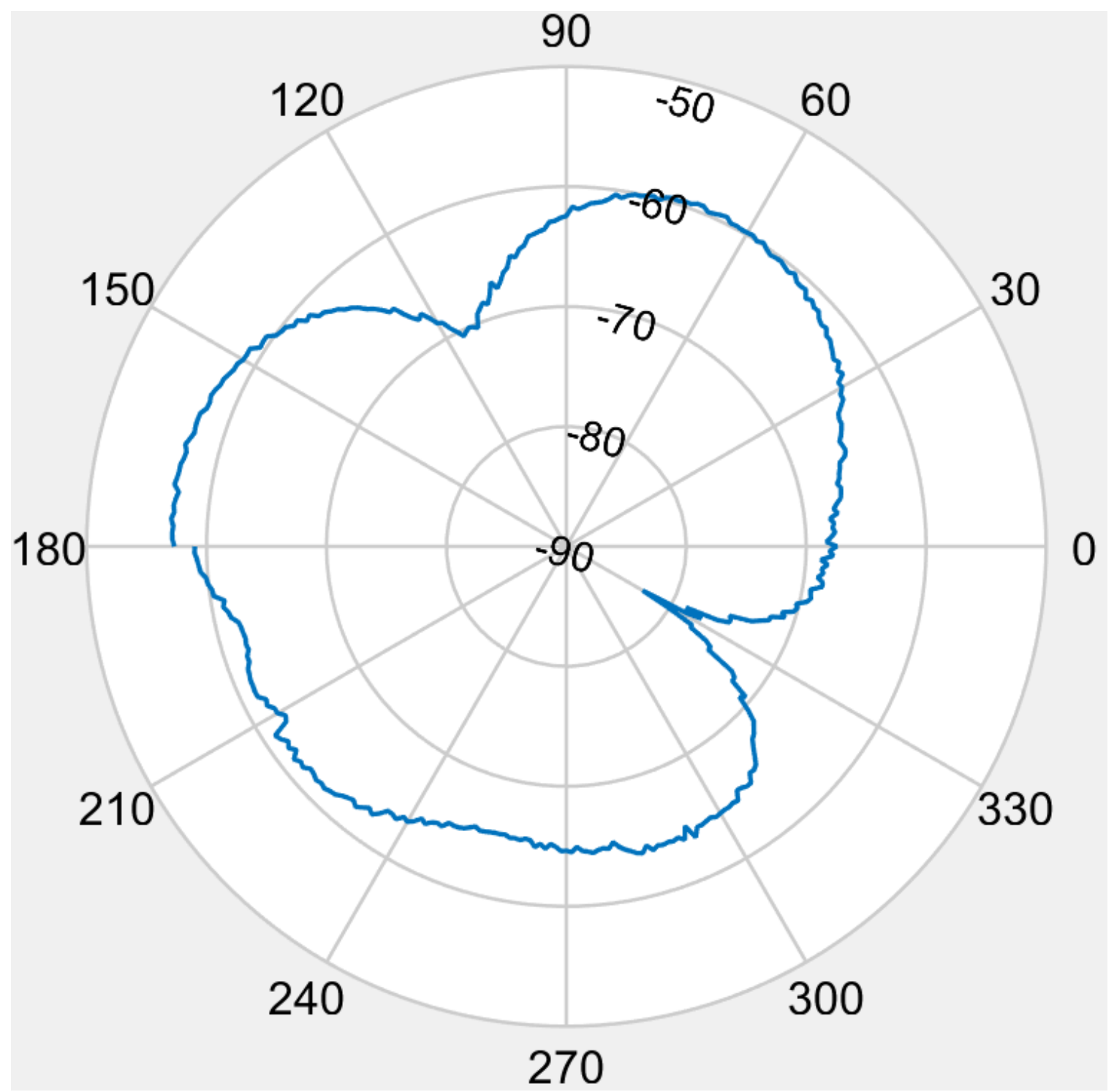

Figure 57: $1 \mathrm{GHz} S 21(d B)$ in azimuth plane for $6 \mathrm{~cm}$ leg length rhombic 


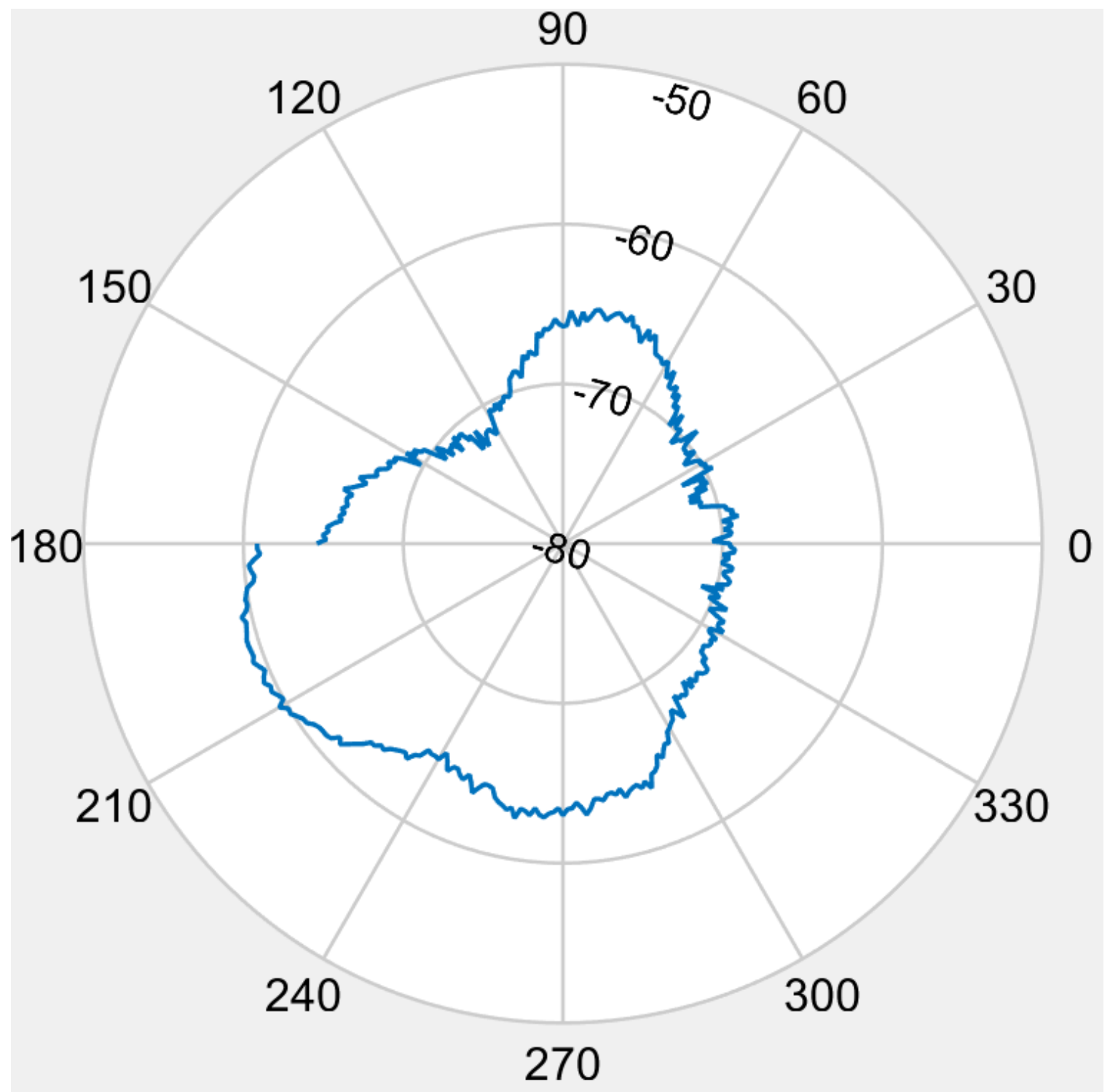

Figure 58: 1.2 GHz S21 $(d B)$ in azimuth plane for $6 \mathrm{~cm}$ leg length rhombic 


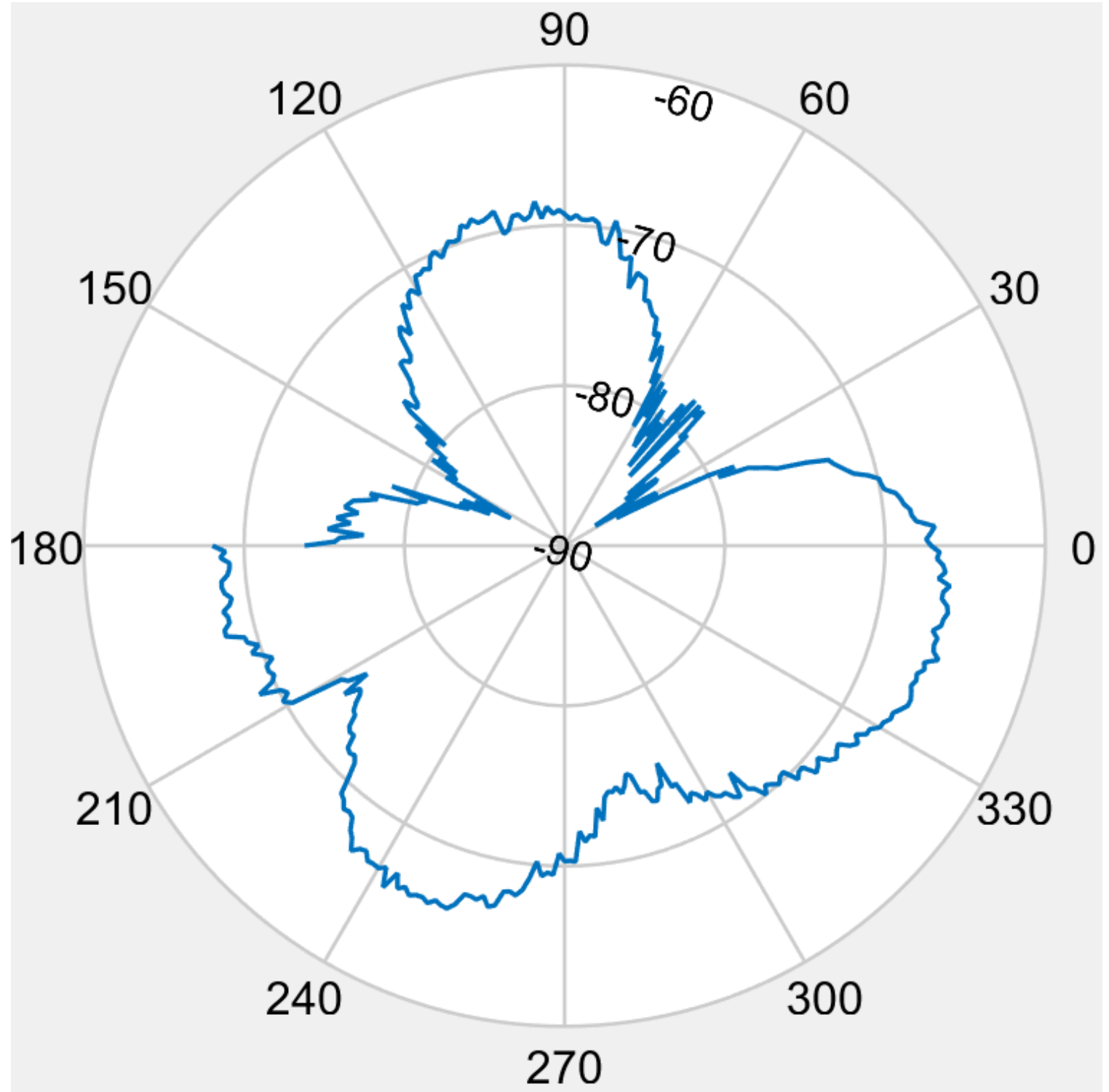

Figure 59: $1.4 \mathrm{GHz} S 21(d B)$ in azimuth plane for $6 \mathrm{~cm}$ leg length rhombic 


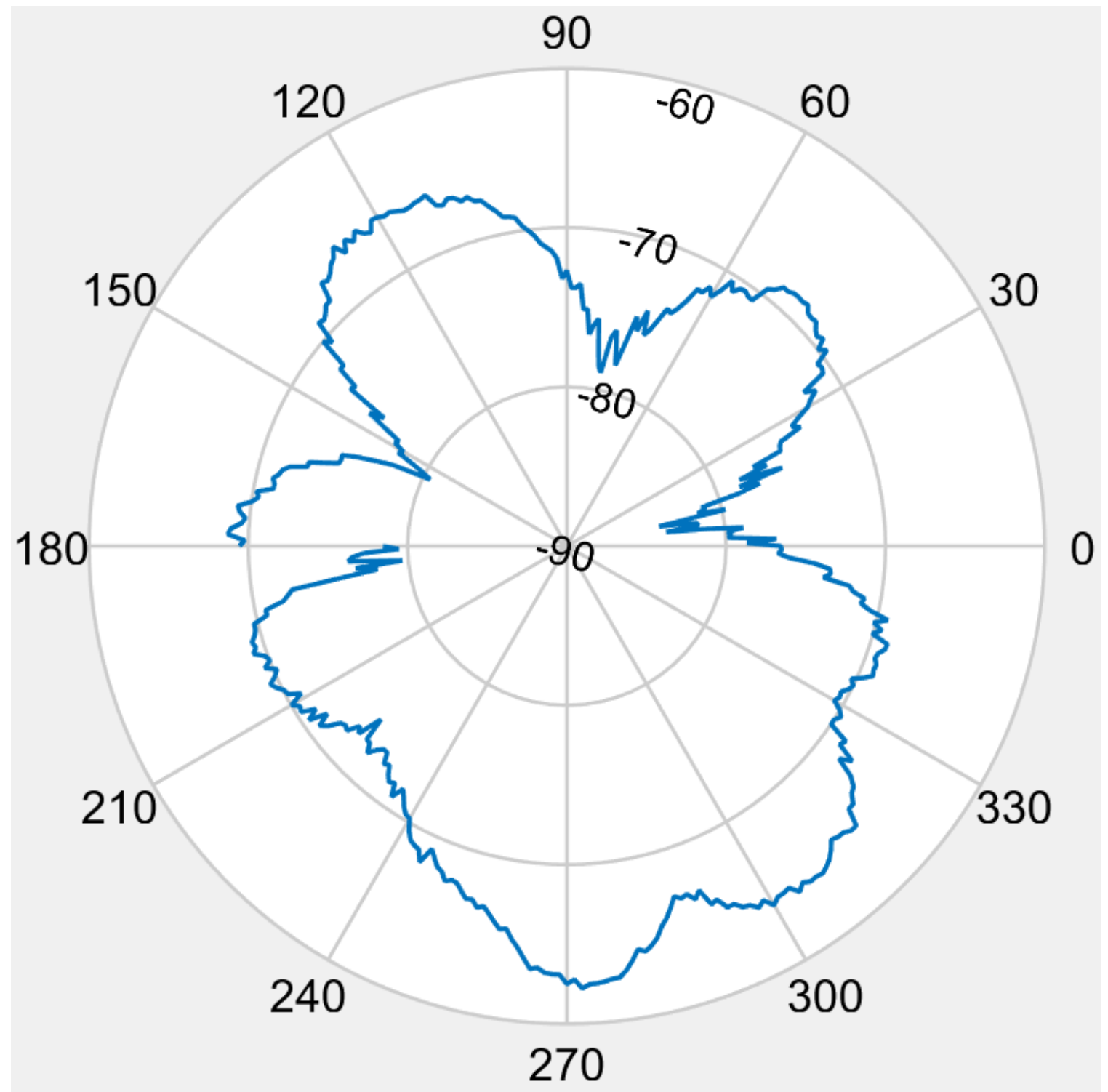

Figure 60: 1.6 GHz S21 (dB) in azimuth plane for $6 \mathrm{~cm}$ leg length rhombic 


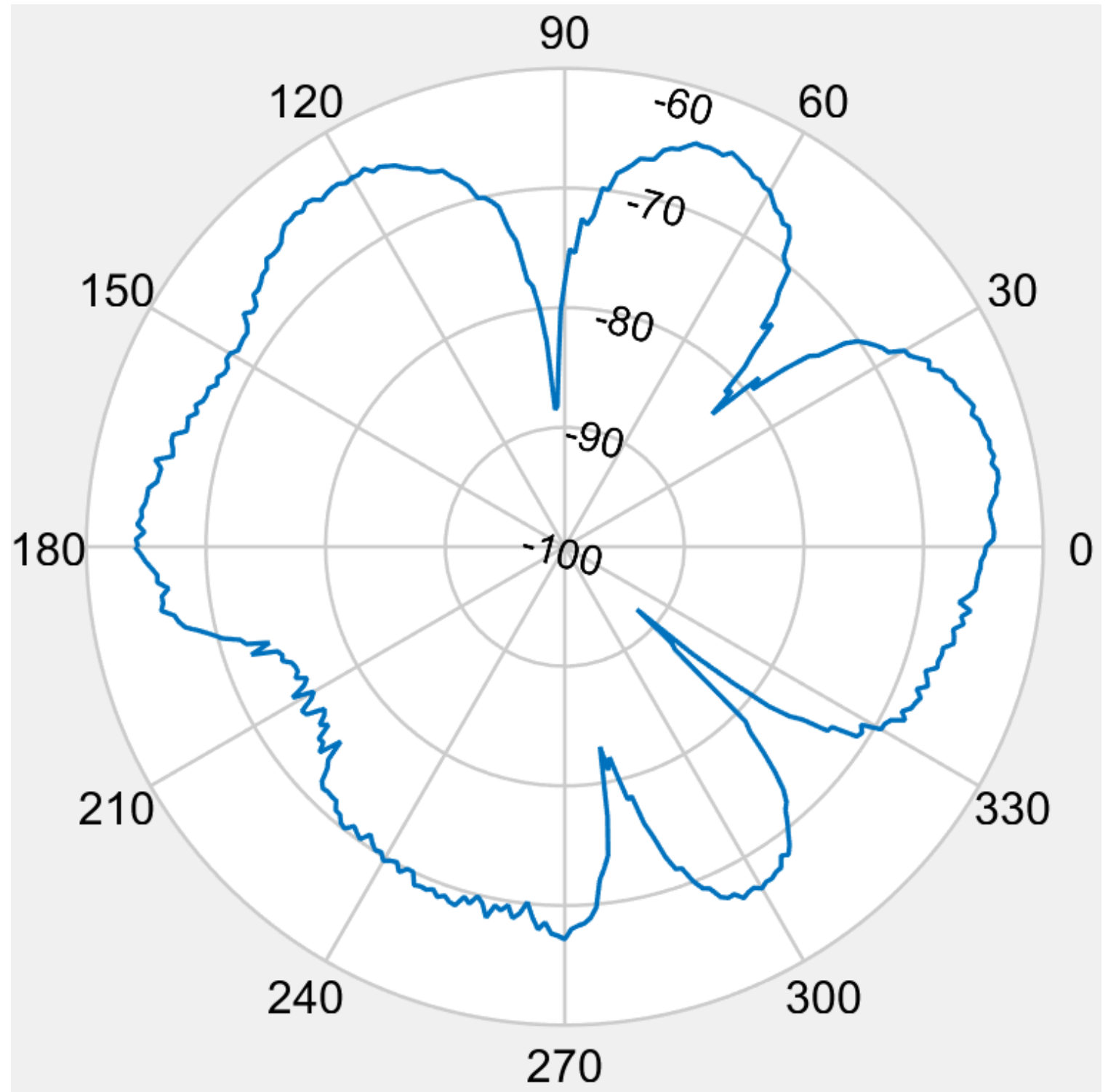

Figure 61: $1.8 \mathrm{GHz} S 21(d B)$ in azimuth plane for $6 \mathrm{~cm}$ leg length rhombic 


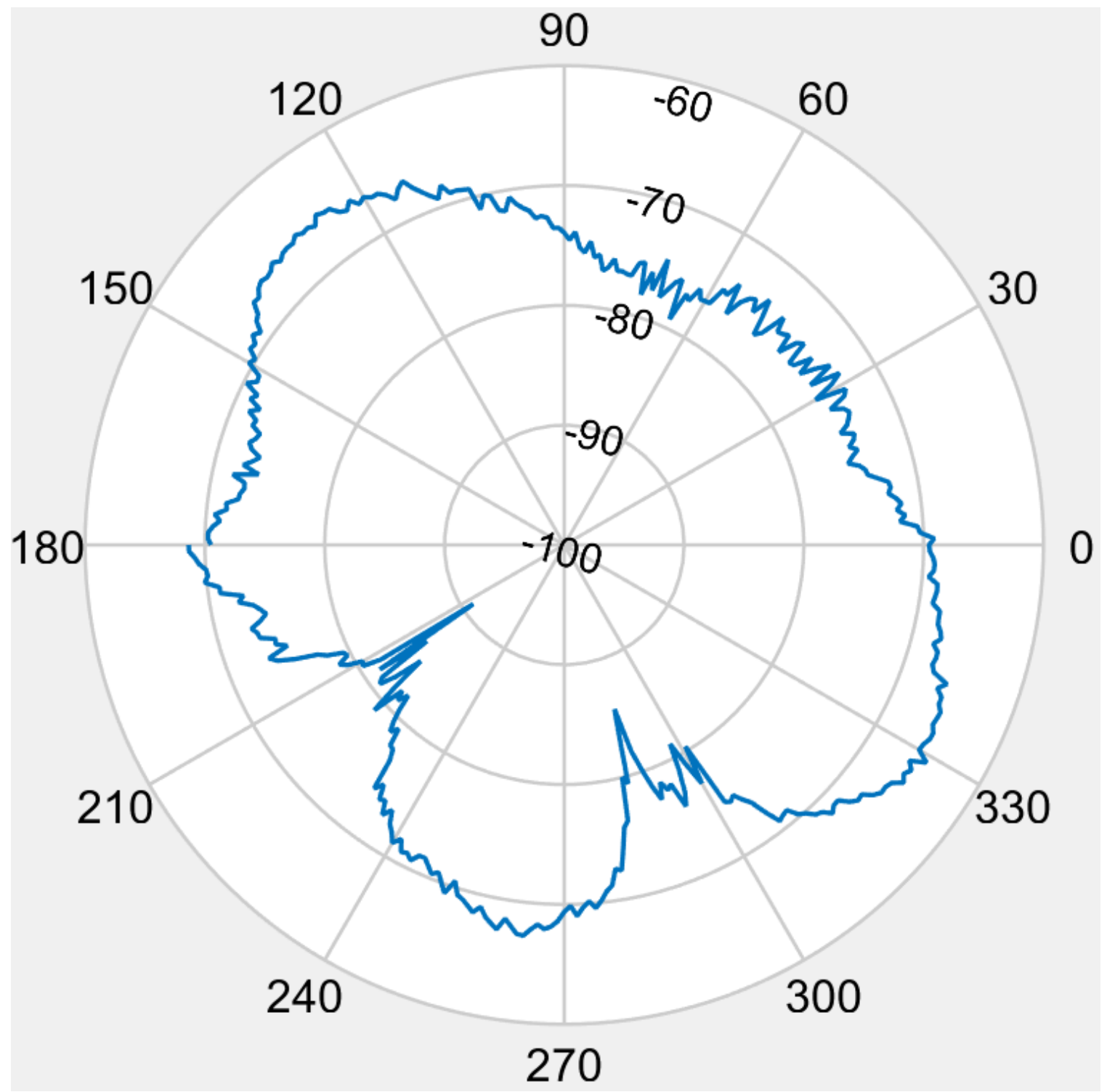

Figure 62: $2 \mathrm{GHz} S 21(d B)$ in azimuth plane for $6 \mathrm{~cm}$ leg length rhombic

When comparing these results to the radiation patterns in the simulation section shown in Appendix A, it can be seen that unwanted sidelobes are being introduced at lower frequencies than anticipated. This indicates that the rhombic leg length should be decreased further in order to remove the sidelobes appearing in the measured S21 patterns as low as $1.4 \mathrm{GHz}$. This will be left as future work as it is not necessary for the DF simulations, which are be performed at $0.8 \mathrm{GHz}$. 
A gain plot is not done for this band since gain standard measurements are not possible using Carleton's anechoic chamber for the majority of this frequency band.

\subsection{DF Simulations with Assembled Rhombic Measurements}

After the rhombic measurements were complete, MATLAB DF simulations were performed with the data. The simulations that were done in the HFSS simulation section are repeated with the measurements taken in this section (i.e. the same process in section 3.4). The DF simulations used measurements taken at $0.8 \mathrm{GHz}$ for the $6 \mathrm{~cm}$ leg length rhombic. Note that no noise or path loss is artificially added with MATLAB since the rhombic measurements were taken in Carleton's anechoic chamber with equipment and cables that inherently add error and noise to the measurements.

The curve shown in the following figure contains the error signal from the S21 data with one rhombic facing at a 22.5 degree offset in relation to the transmitting antenna in the chamber and the other shifted 45 degrees to match the design of the final UAS DF product. 


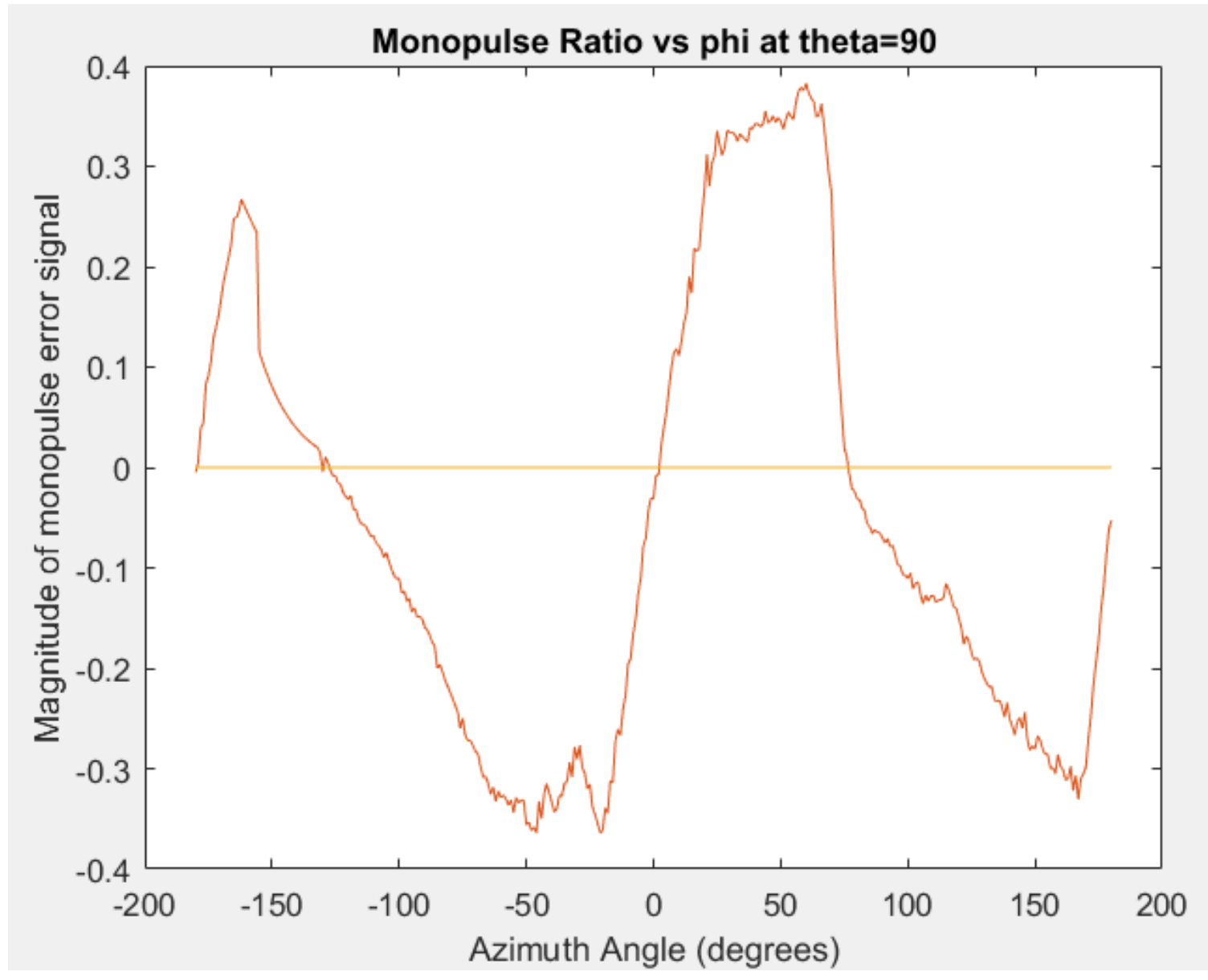

Figure 63: Error signal for $6 \mathrm{~cm}$ leg length rhombic vs phi at $0.8 \mathrm{GHz}$

Another plot showing a zoomed in version of the error signal is shown below: 


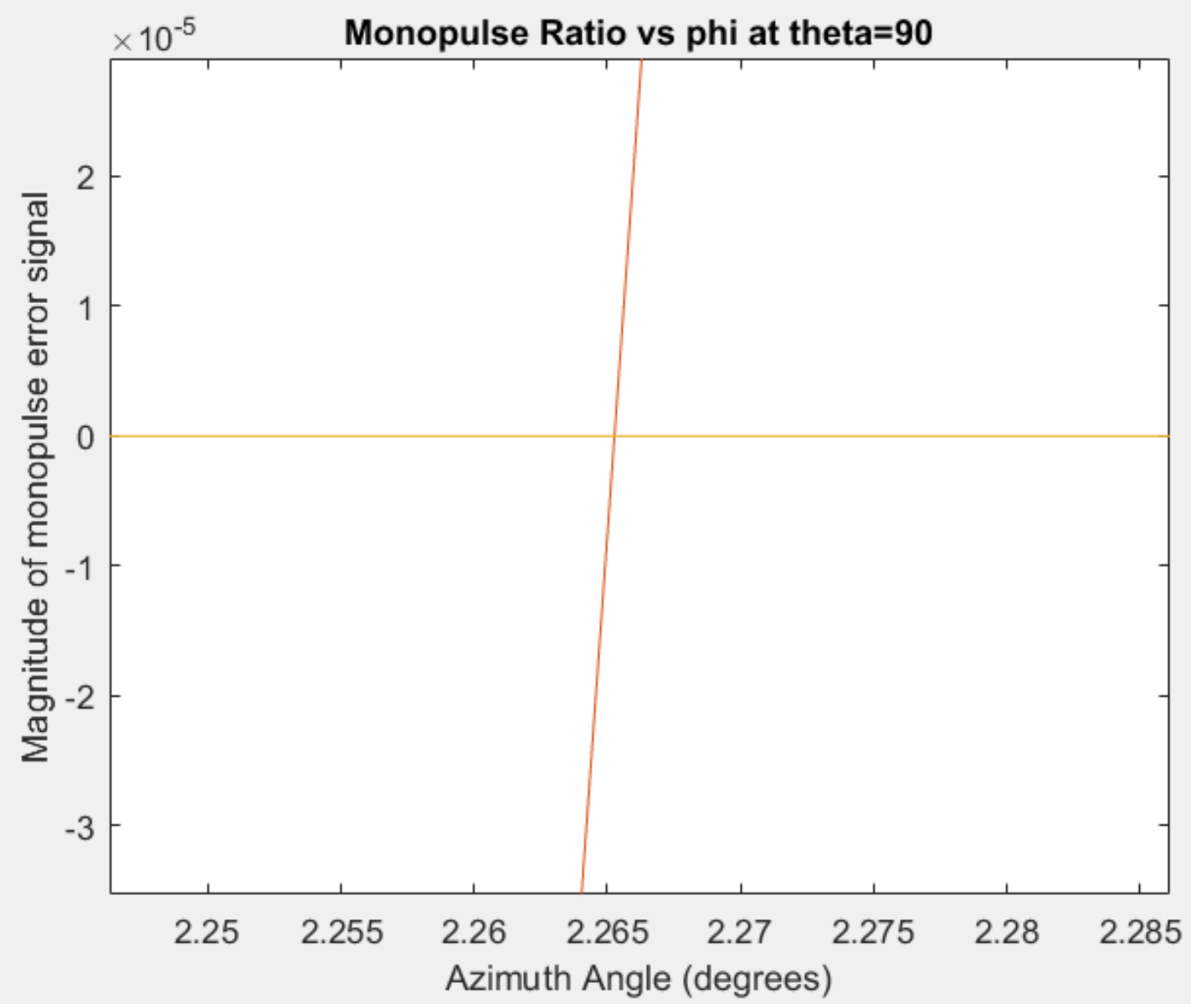

Figure 64: Zoomed in error signal of $6 \mathrm{~cm}$ leg length rhombic vs phi at $0.8 \mathrm{GHz}$

It can be seen from the plot above that the target (transmitter in the anechoic chamber in this case) is approximately 2.265 degrees in the azimuth direction relative to the rhombics. The transmitter was setup in the anechoic chamber along the tracking axis of the receiving rhombic, giving an error of approximately 2.265 degrees. A table of results is given below at 5 degree rhombic incremental azimuth offsets from -15 degrees to 15 degrees in the anechoic chamber. 
Table 4: Monopulse Error Results from -15 to $15^{\circ}$ rhombic offsets in the azimuth plane

\begin{tabular}{|c|c|c|}
\hline Receiver Bearing $\left({ }^{\circ} \mathrm{AZ}\right)$ & $\begin{array}{c}\text { Predicted Location by } \\
\text { Amplitude Comparison } \\
\text { Monopulse }\left({ }^{\circ} \mathrm{AZ}\right)\end{array}$ & $\begin{array}{c}\text { Error in Monopulse } \\
\text { Prediction }\left({ }^{\circ} \mathrm{AZ}\right)\end{array}$ \\
\hline-15 & -18.545 & 3.545 \\
\hline-10 & -12.369 & 2.369 \\
\hline-5 & -1.838 & 3.162 \\
\hline 0 & 2.265 & 2.265 \\
\hline 5 & 2.3957 & 2.6043 \\
\hline 10 & 6.726 & 3.274 \\
\hline 15 & 18.343 & 3.343 \\
\hline
\end{tabular}

This is slightly better bearing accuracy than expected. In the amplitude comparison monopulse section, errors for a similar system were around 6.12 degrees. While it was expected for the theoretical error to be significantly better than 6.12 degrees, it was not expected to be this much better across a spread of transmitter locations. This is likely due to a lack of a polarization mismatch and potentially some small experimental errors such as the mechanical turntable's turning error working in the favour of this method. The accuracy of this method could be improved slightly by further optimizing the rhombic antennas radiation patterns. As mentioned in Chapter 4, this can be done by using a larger UAV which can allow more sets of rhombics to be used in order to more closely follow the designed squint angle for the large $0.6-6 \mathrm{GHz}$ frequency range. Alternatively, the frequency range requirement could be reduced, which would accomplish the same effect. 


\section{6 - Conclusion and Future Work}

\subsection{Conclusion}

The open loop amplitude comparison monopulse DF technique using rhombic antennas yielded an experimental accuracy between 2.265 and 3.545 degrees. This is a notable improvement over current DF UAS' which can currently obtain an accuracy around 10 degrees using conventional modern DF techniques with a UAS. These results would also indicate that rhombic antennas are suitable to use with amplitude comparison monopulse as a DF technique from 0.6-6 GHz. It is expected that in practical cases with multiple rhombics mounted on a UAS the bearing error could be significantly worse than this. The bearing error should still be a significant improvement upon current DF techniques currently implemented with UAS' and should be well below the target maximum allowed bearing error of 6.12 degrees.

The rhombic antennas were simulated using HFSS and tested using Carleton University's anechoic chamber. HFSS simulation results and antenna test results were then imported into MATLAB and relevant monopulse error signals were plotted using MATLAB's antenna and phased array toolboxes. The rhombics were found to have a reasonable gain with HPBW's suitable for use with open loop amplitude comparison monopulse. The leg lengths used for these rhombics were $2 \mathrm{~cm}$ for a frequency range from 2-6 GHz and $6 \mathrm{~cm}$ for a frequency range from $0.6-2 \mathrm{GHz}$.

The advantages of this technology are not limited to higher DF accuracy. Since rhombic antennas are wire antennas, they are not greatly affected by changes in temperature. This can allow them to be used in different environments without needing calibration. This lowers maintenance and training costs for those who would choose to implement this 
technology when compared to other DF technologies. In addition to this, the UAS itself is inherently a low cost implementation which would be very attractive to a government or company looking to mass produce a mobile airborne DF system.

\subsection{Future Work}

From the results of this thesis there are a few things that a future researcher could investigate to improve the open loop amplitude comparison monopulse bearing error in a UAS. These include manufacturing and testing etched rhombic antennas in a two channel system, investigating the effects of changing the apex angle of the rhombic antennas, using larger UAV's which can carry larger rhombics, and test a DF UAS several thousand feet in the air.

Since this thesis was done as a proof of concept for the use of open loop amplitude comparison monopulse in a UAS with rhombic antennas, a future researcher could focus on building this system by integrating the required components and testing the system. To build and test this UAS, a future researcher could manufacture etched rhombic antennas and test them in an anechoic chamber with a two channel receiver to observe the effects of mutual coupling between the rhombics at different frequencies. Additionally, the rhombics could be integrated with a two channel receiver on a UAV and flown at different altitudes to confirm whether or not there is any noticeable difference in bearing error. It is important to note that if a future researcher were interested in building this system a method of switching between rhombics would be required such that the upper band rhombics are only used in the upper band, and the lower band rhombics are only used in the lower band. 
The rhombic antenna configuration could potentially be further optimized by a future researcher. As mentioned in Chapter 4, if a UAV larger than the UAV selected for this thesis were used, the S11 and gain of the rhombic antennas could be improved. These characteristics can be improved since more rhombics could be carried by the UAV and therefore the rhombics would operate in smaller bandwidths. The apex angle of the rhombics could potentially make a small impact on the bearing error as well since it correlates to their HPBW and optimum squint angle for amplitude comparison monopulse.

If these tasks were completed, this thesis research could result in a high-end DF UAS at a low cost. 


\section{References}

[1] UCAR Center for Science Education, "Layers of Earth's Atmosphere," 2015.

[Online]. Available: https://scied.ucar.edu/atmosphere-layers. [Accessed: Oct. 11 ${ }^{\text {th }}, 2017$ ]

[2] Tactical Imaging Systems. (ND). Exocet all weather low cost VTOL UAV UAS Unmanned drone with EOIR Gyro Stabilized Thermal FLIR HD Zoom Gimbal Turret Payload [Online]. Available: http://inkwebstudio.com/tactical/exocet-all-weather-lowcost-vtol-uav-uas-unmanned-drone-with-eoir-gyro-stabilized-thermal-flir-hd-zoomgimbal-turret-payload/

[3] E.A. Laport, "Horizontal Rhombic Antennas," in Radio Antenna Engineering, New York: Mcgraw Hill, 1952, ch. 3, sec 23, pp. 315-339

[4] Rohde \& Schwarz. (ND). Introduction into Theory of Direction Finding [Online]. Available: http://telekomunikacije.etf.bg.ac.rs/predmeti/ot3tm2/nastava/df.pdf

[5] M.I. Skolnik, "Monopulse Tracking", in Introduction to Radar Systems, $3^{\text {rd }}$ ed. New York, NY: McGraw Hill Inc., 2001, ch.4, sec.2, pp. 213-224

[6] S.M Sherman, "Monopulse Principles and Techniques," Dedham, MA, 1984, Artech House Inc., ch. 2, sec 4-6, pp. 29-33

[7] D.K. Barton, "Radar System Analysis and Modeling," Boston, MA, 2005, Artech House Inc., ch. 8, sec 2.4, pp.393-400

[8] M.I. Skolnik, "Monopulse Tracking Radar" in Introduction to Radar Systems, $2^{\text {nd }}$ ed. New York, NY: McGraw Hill Inc., 1980, ch.5, sec.4, pp.160-167.

[9] Y.X. Zhang. (2016, June 3). A Novel Monopulse Angle Estimation Method for Wideband LFM Radars [Online]. Available: http://www.mdpi.com/1424$\underline{8220 / 16 / 6 / 817 / \mathrm{htm}}$

[10] L.G. Bullock. (1970, Aug. 25). An Analysis of Wide-Band Microwave Monopulse Direction-Finding Techniques [Online]. Available: http://ieeexplore.ieee.org/document/4103675/

[11] Earth and Environmental Sciences Columbia University, "Lapse Rate, Moisture, Clouds, and Thunderstorms." Columbia University, 2007. [Online]. Available: http://eesc.columbia.edu/courses/ees/climate/lectures/atm_phys.html. [Accessed: Oct. $\left.11^{\text {th }}, 2017\right]$

[12] Hargrave, Frank. (01/01/2001). Rhombic antenna. Hargrave's Communications Dictionary: Basic Terms, Equations, Charts and Illustrations. 
[13] C.A. Balanis, "Travelling Wave and Broadband Antennas" in Antenna Theory Analysis and Design. New York, NY: Harper \& Row, 1982, sec.3, pp.383-385

[14] S.K. Singh, N. Singh, and G. Varshney, "Inductive Coupling Effect on The Radiation Pattern Of V-Shaped Antenna Array," International Journal of Emerging Technology and Advanced Engineering, vol. 4, no. 1, February 2014, [online document], Available:

https://www.researchgate.net/publication/275954753_Inductive_Coupling_Effect_on_Th e_Radiation_Pattern_Of_V-Shaped_Antenna_Array [Accessed November $8^{\text {th }}, 2017$ ].

[15] S.W. Kershner, "High Frequency Arrays," in Antenna Engineering Handbook, $1^{\text {st }}$ ed. New York, NY: McGraw-Hill Inc., 1961, ch.21, sec.4, pp.21-17 ->21-23.

[16] P.J. Bevelacqua, "S-Parameters," 2015. [Online]. Available: http://www.antennatheory.com/definitions/sparameters.php [Accessed: November $7^{\text {th }}, 2017$ ]

[17] Electro-Metrics, "6961-Performance-Data", N.D. [Online]. Available:

https://electro-metrics.com/wp-content/uploads/2017/04/6961-Performance-Data.pdf

[Accessed: July 30 ${ }^{\text {th }}, 2017$ ]

[18] C.H. Walter, "Practical Travelling Wave Antennas" in Travelling Wave Antennas. New York, NY: Dover Publications Inc., 1965, sec.2c, pp. 319-321 


\section{Appendices}

Appendix A: Lower Frequency Band Radiation Pattern Simulations

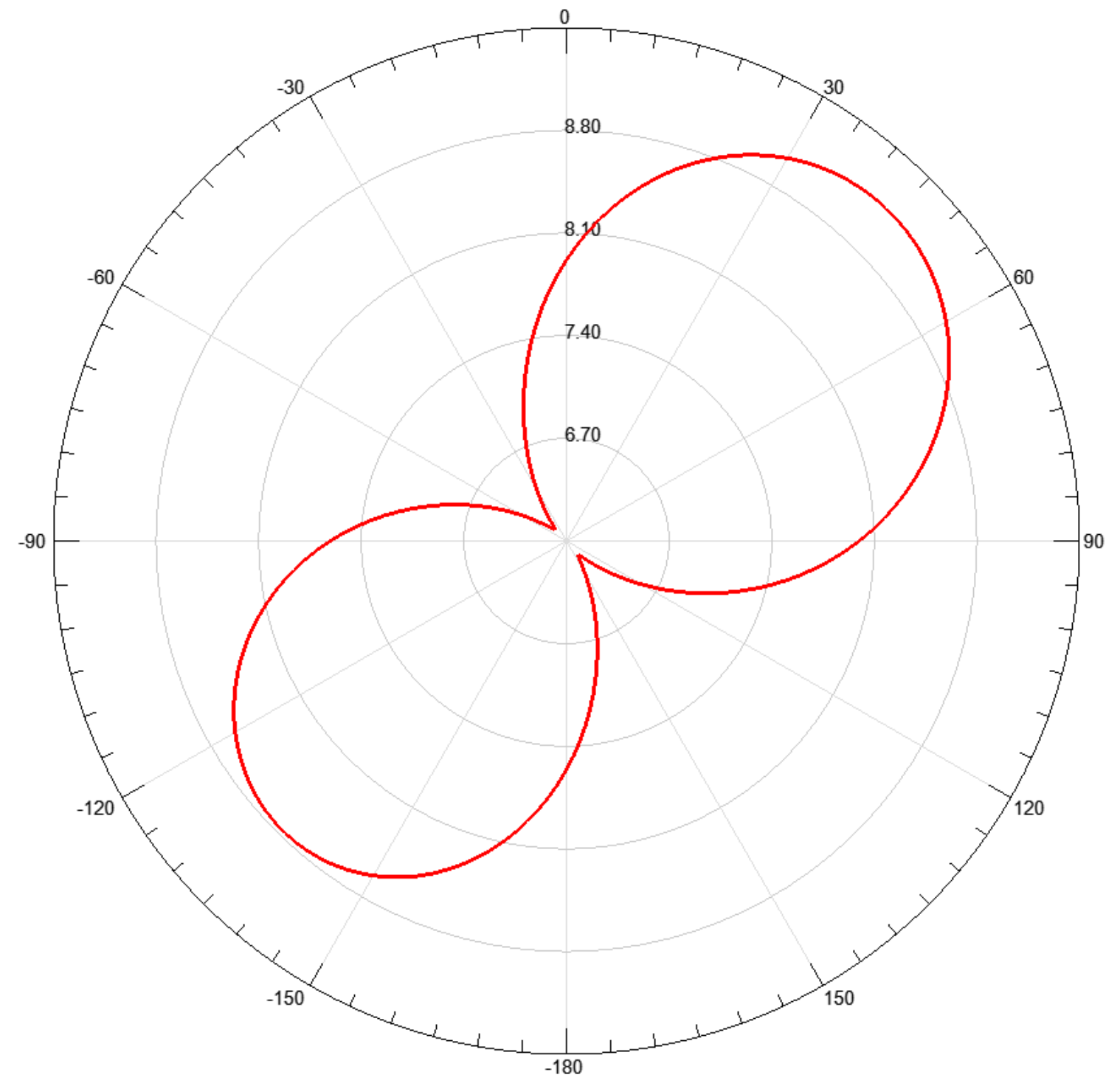

Figure 65: $6 \mathrm{~cm}$ leg length at $0.6 \mathrm{GHz}$ with $2 \mathrm{~mm}$ wire thickness and $1 \mathrm{~mm}$ separation 


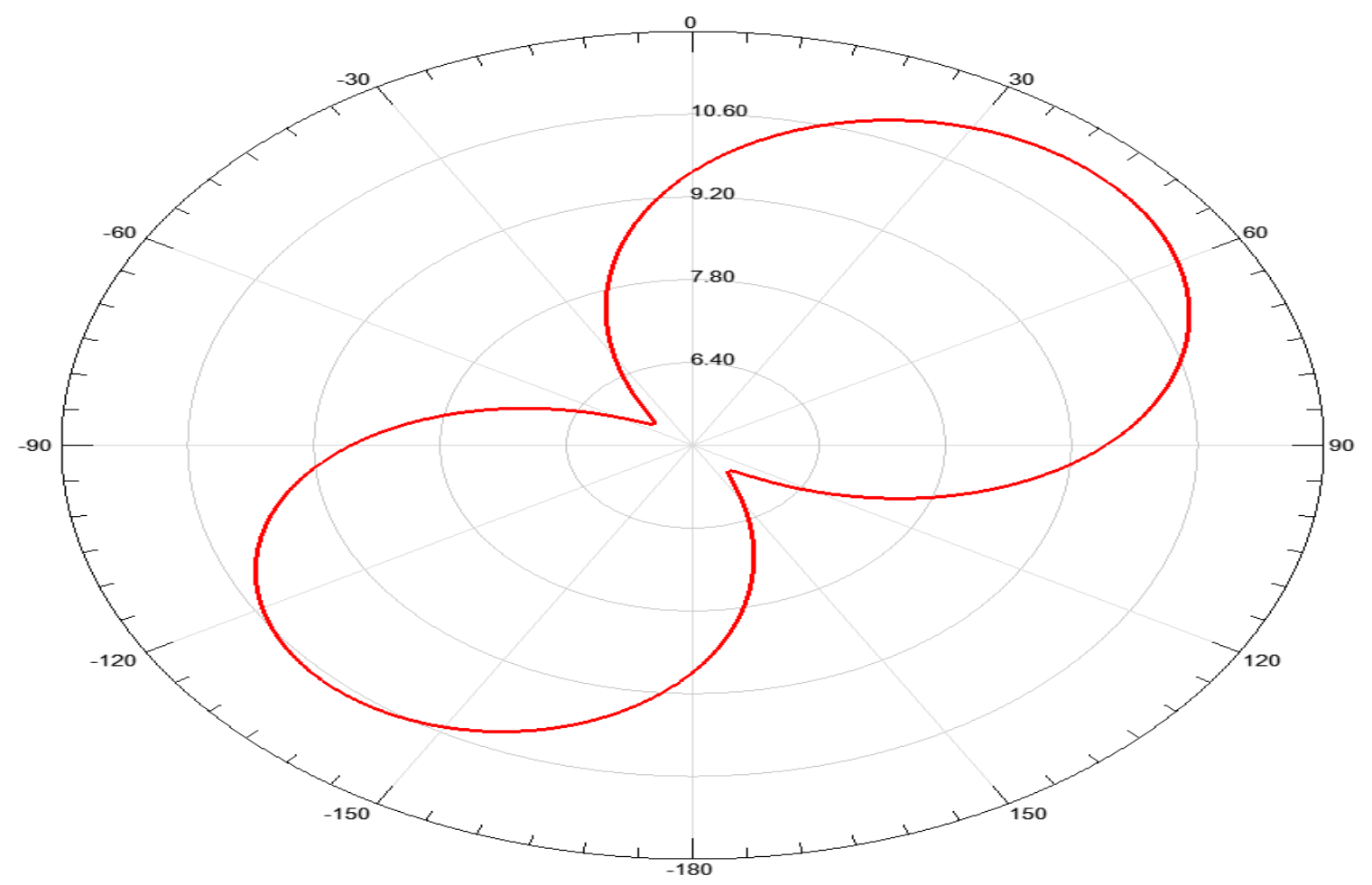

Figure 66: $6 \mathrm{~cm}$ leg length at $0.8 \mathrm{GHz}$ with $2 \mathrm{~mm}$ wire thickness and $1 \mathrm{~mm}$ separation

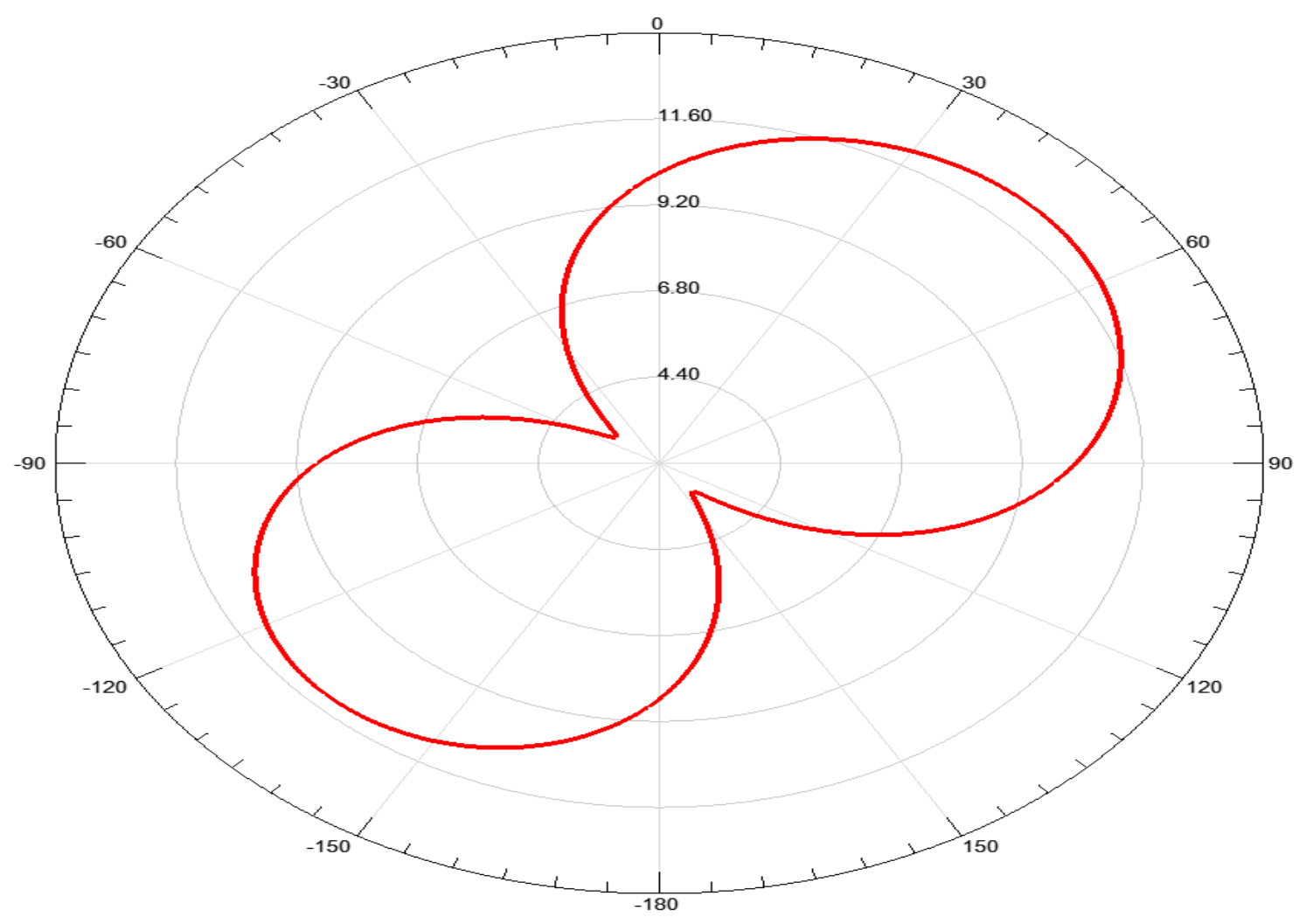

Figure 67: $6 \mathrm{~cm}$ leg length at $1 \mathrm{GHz}$ with $2 \mathrm{~mm}$ wire thickness and $1 \mathrm{~mm}$ separation 


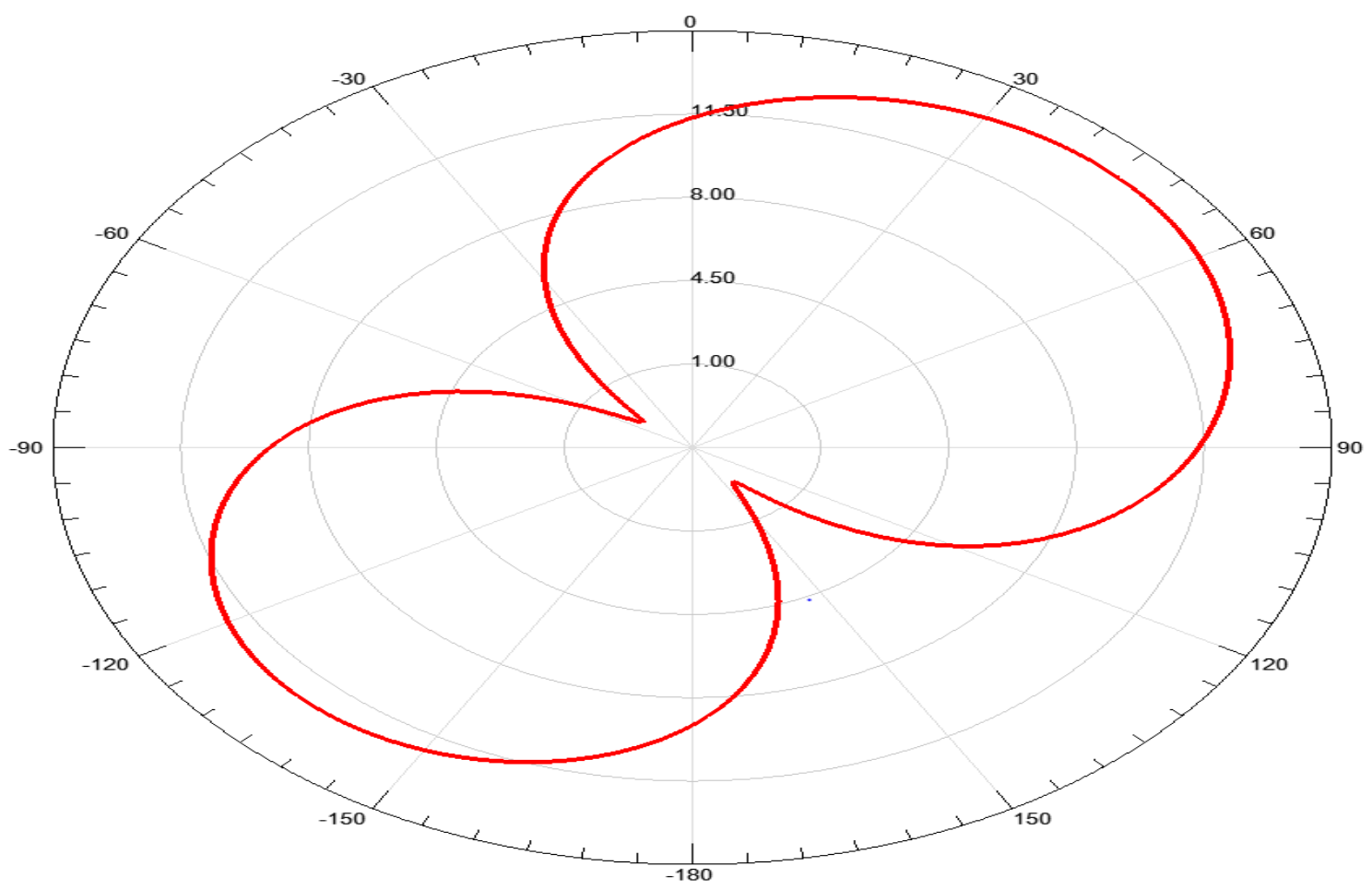

Figure 68: $6 \mathrm{~cm}$ leg length at $1.2 \mathrm{GHz}$ with $2 \mathrm{~mm}$ wire thickness and $1 \mathrm{~mm}$ separation

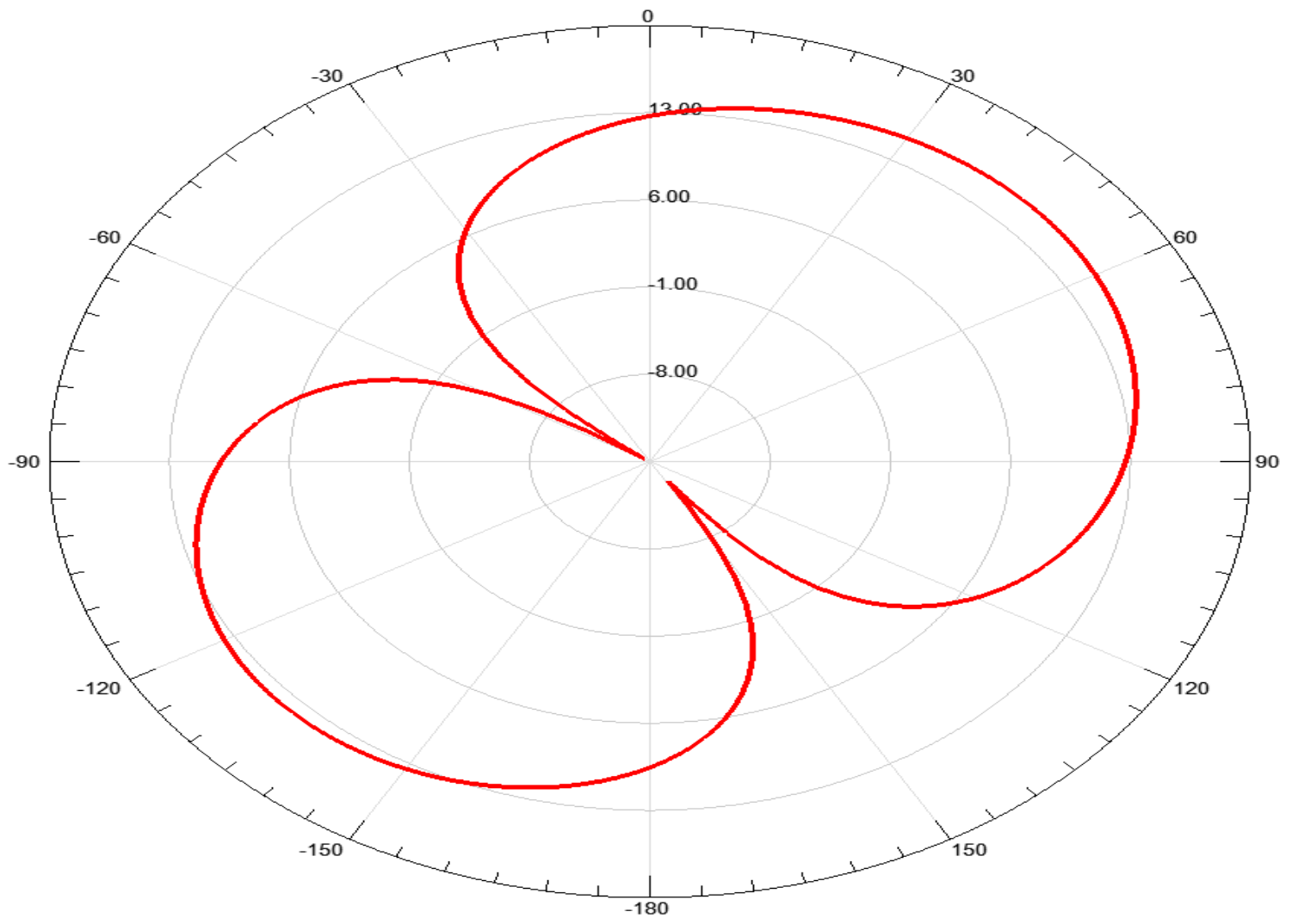

Figure 69: $6 \mathrm{~cm}$ leg length at $1.4 \mathrm{GHz}$ with $2 \mathrm{~mm}$ wire thickness and $1 \mathrm{~mm}$ separation 


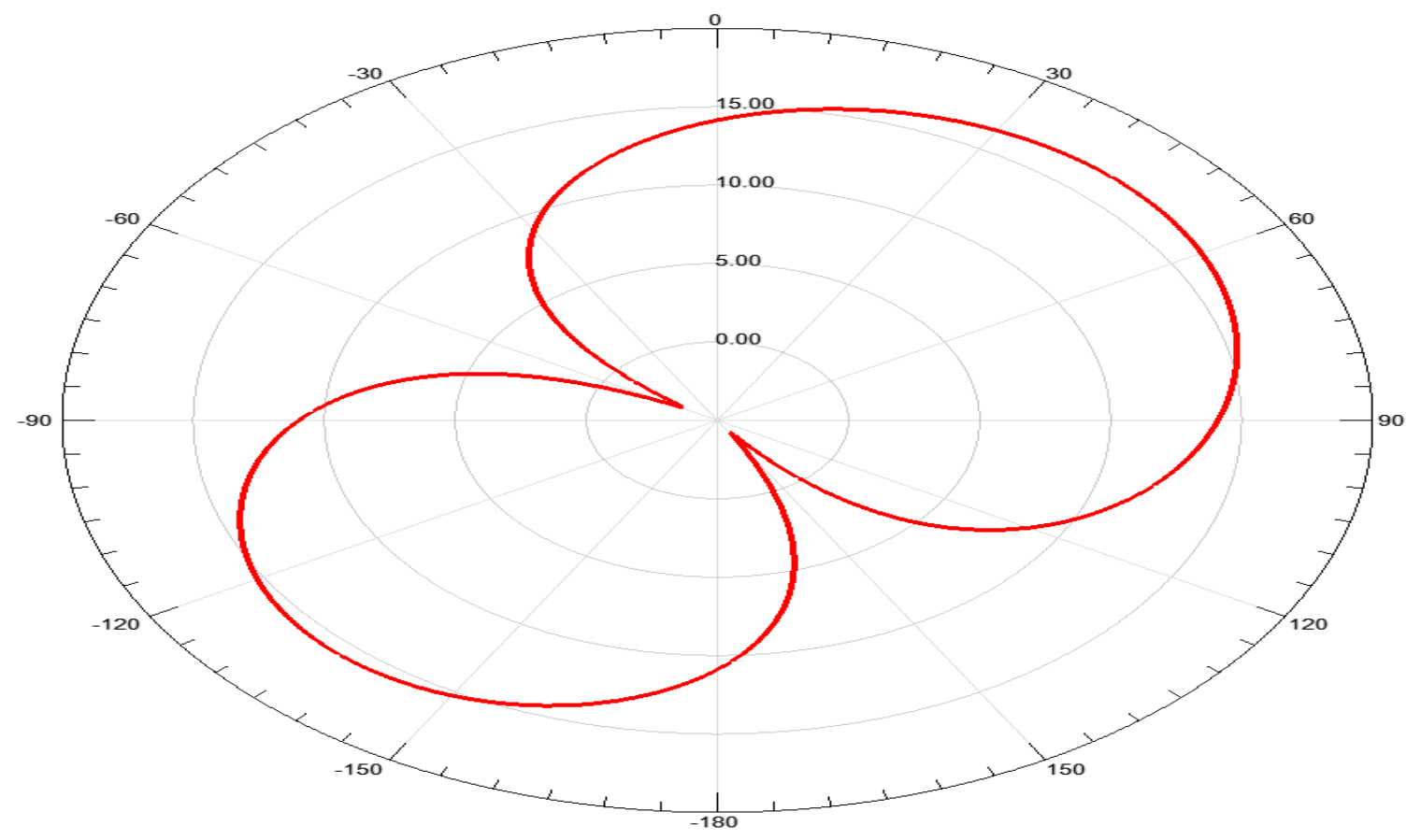

Figure 70: $6 \mathrm{~cm}$ leg length at $1.6 \mathrm{GHz}$ with $2 \mathrm{~mm}$ wire thickness and $1 \mathrm{~mm}$ separation

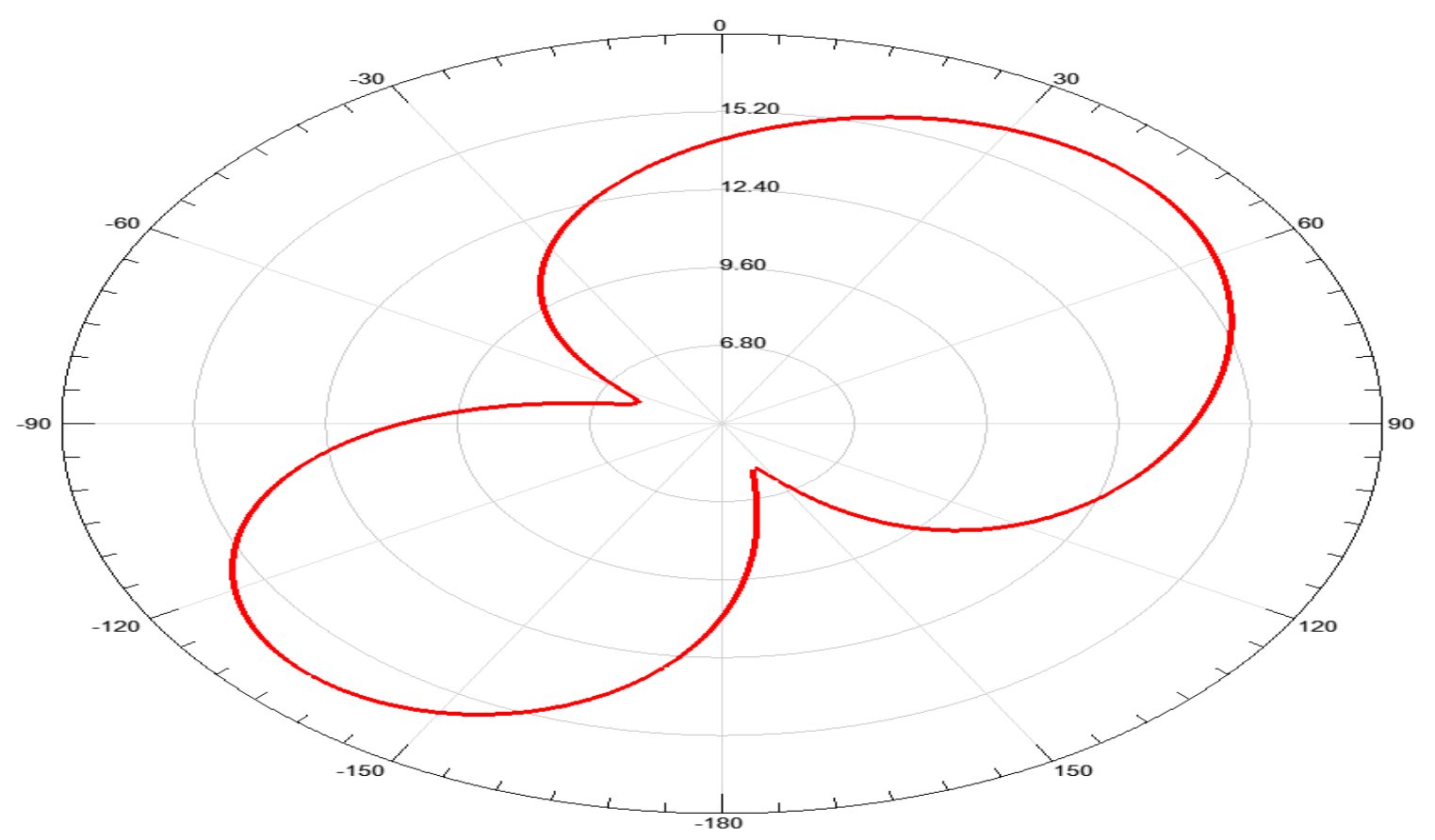

Figure 71: $6 \mathrm{~cm}$ leg length at $1.8 \mathrm{GHz}$ with $2 \mathrm{~mm}$ wire thickness and $1 \mathrm{~mm}$ separation 


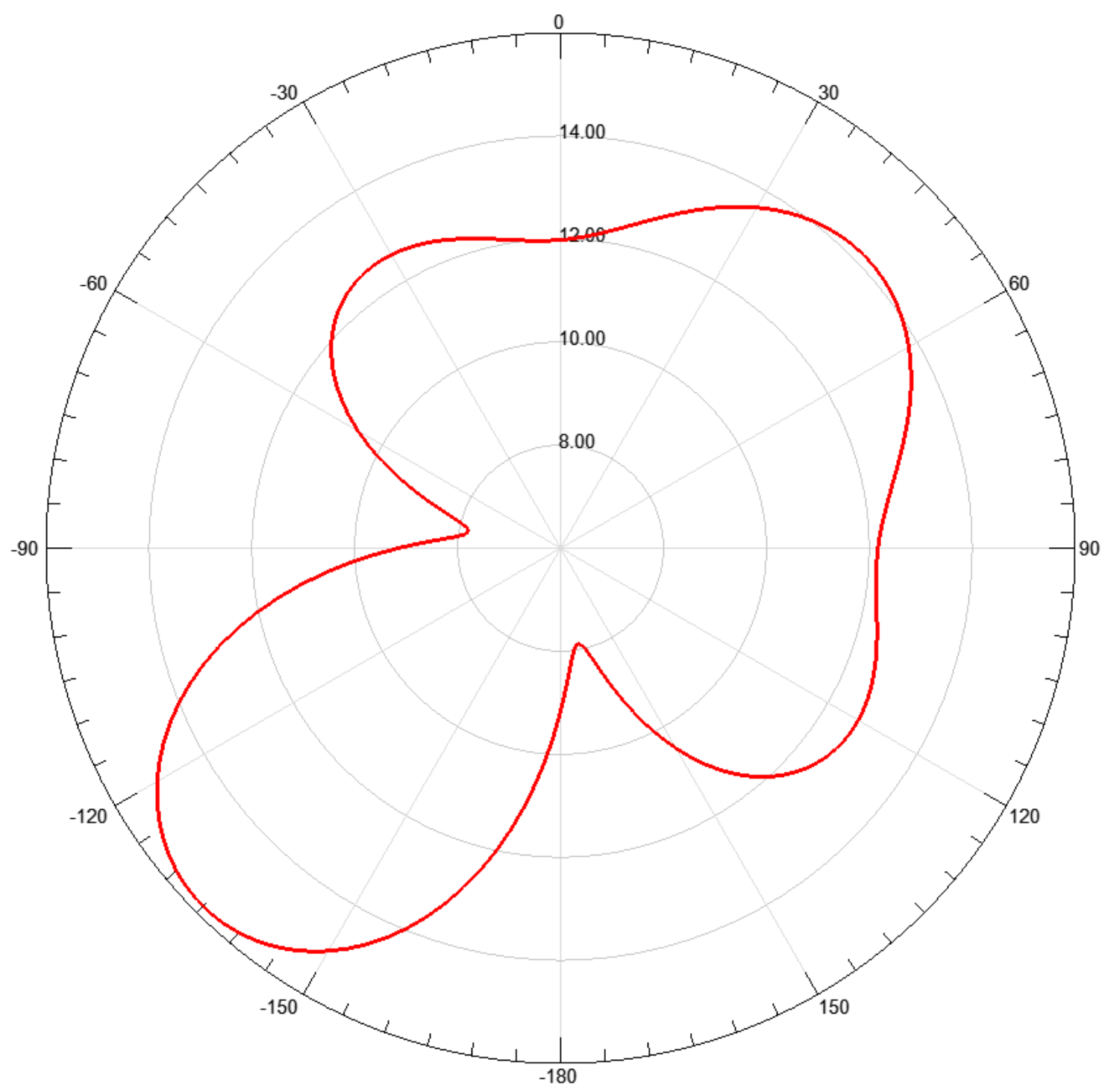

Figure 72: $6 \mathrm{~cm}$ leg length at $2 \mathrm{GHz}$ with $2 \mathrm{~mm}$ wire thickness and $1 \mathrm{~mm}$ separation 


\section{Appendix B: Upper Frequency Band Radiation Pattern Simulations}

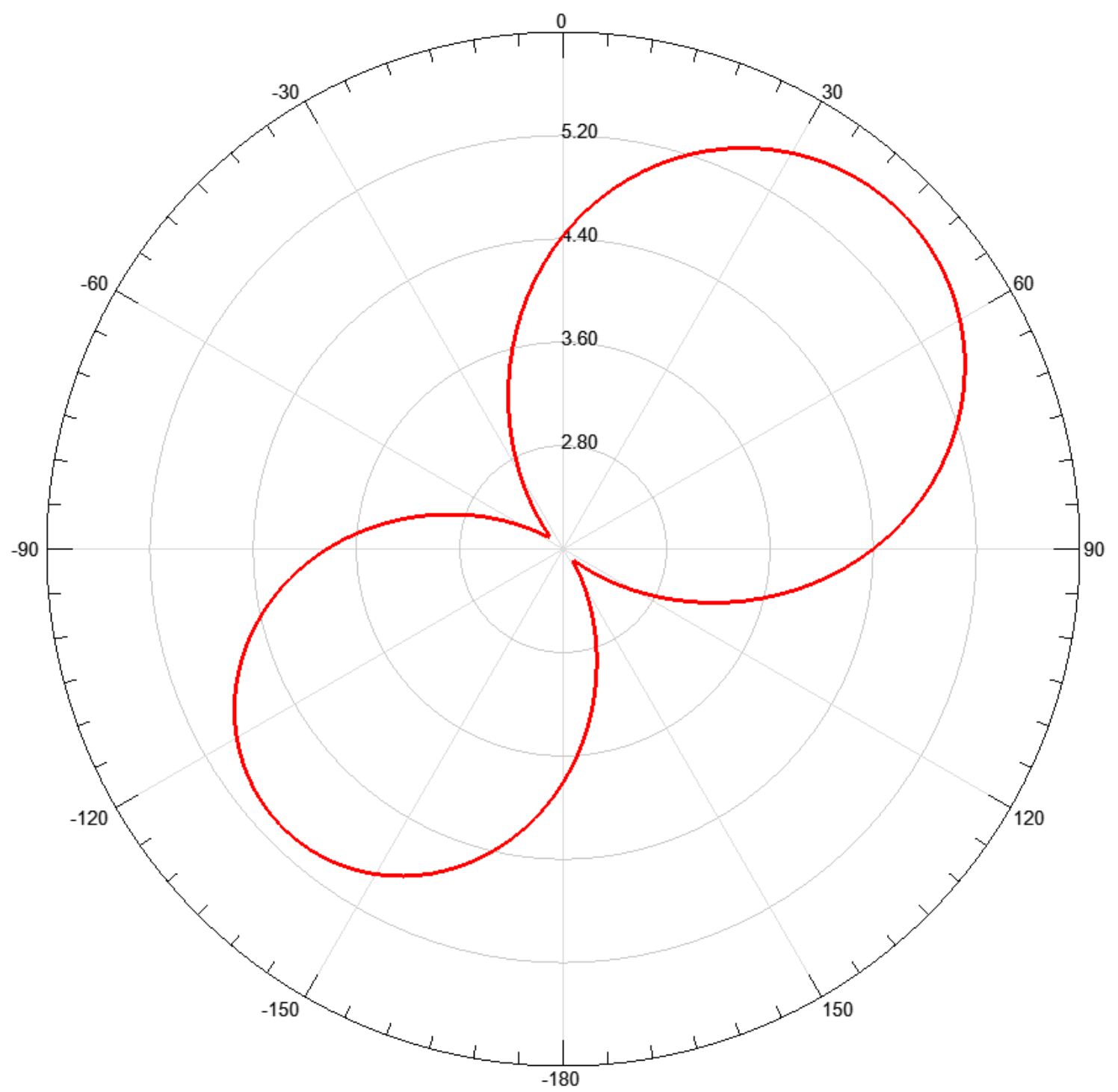

Figure 73: $2 \mathrm{~cm}$ leg length at $2 \mathrm{GHz}$ with $2 \mathrm{~mm}$ wire thickness and $1 \mathrm{~mm}$ separation 


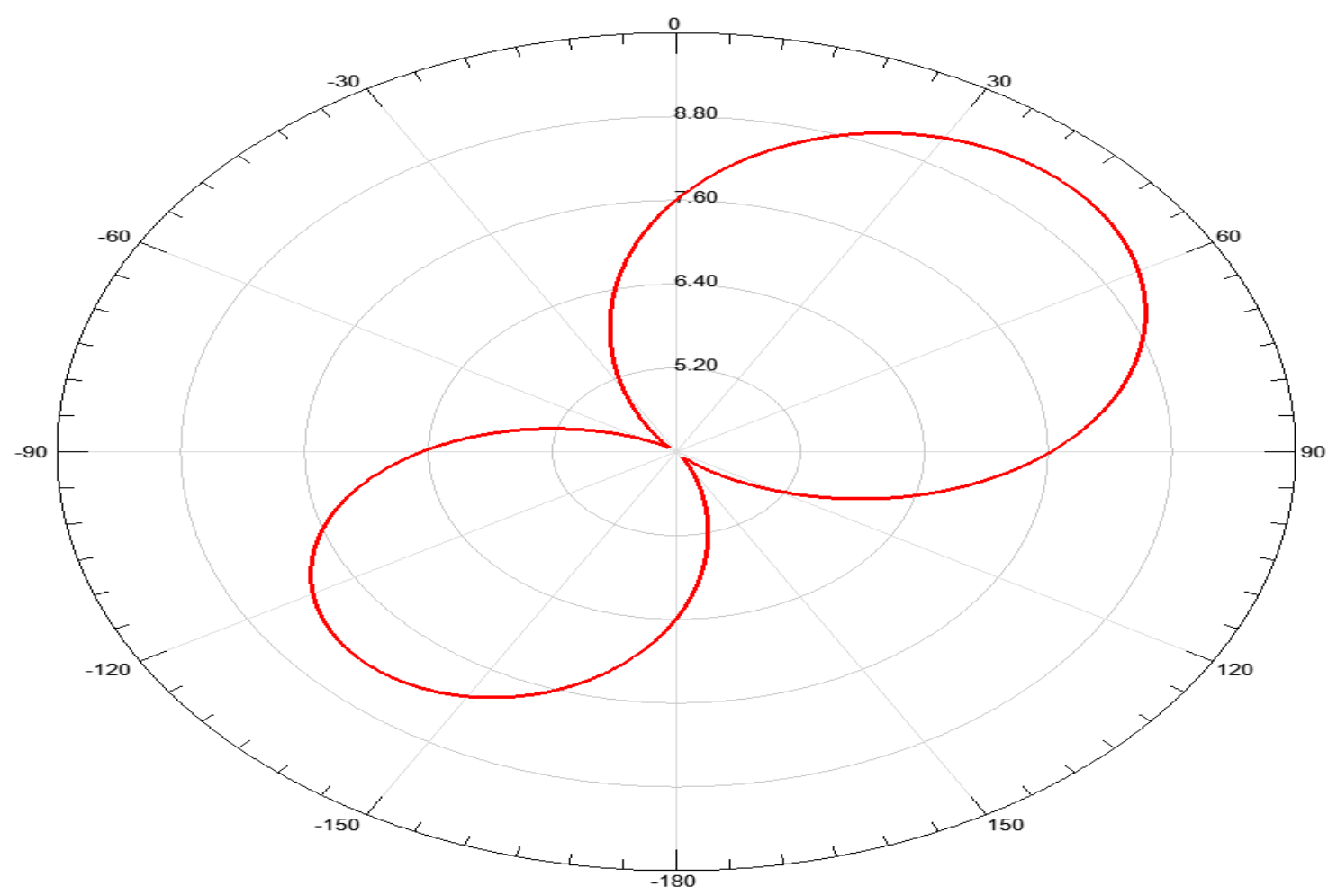

Figure 74: $2 \mathrm{~cm}$ leg length at $2.5 \mathrm{GHz}$ with $2 \mathrm{~mm}$ wire thickness and $1 \mathrm{~mm}$ separation

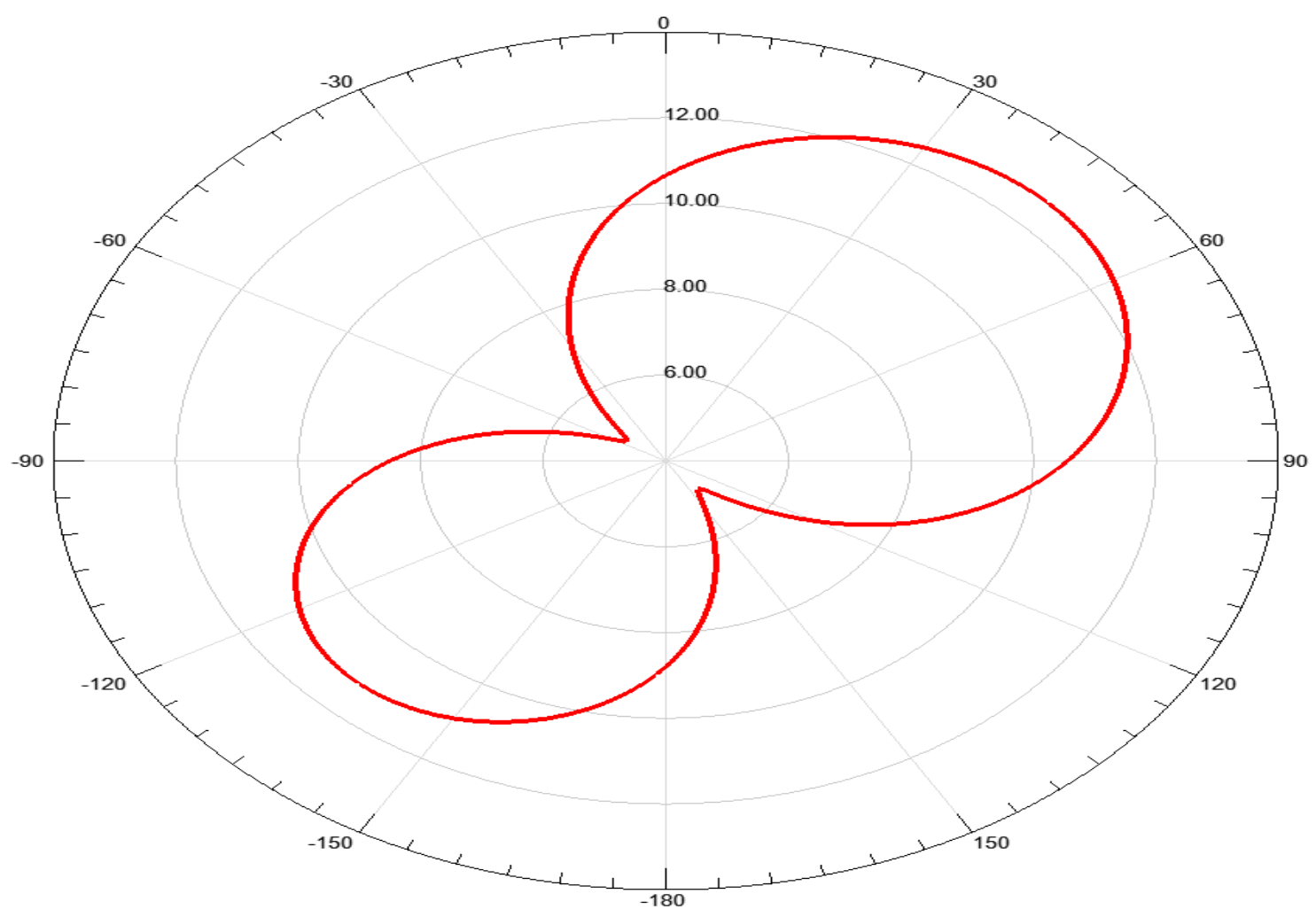

Figure 75: $2 \mathrm{~cm}$ leg length at $3 \mathrm{GHz}$ with $2 \mathrm{~mm}$ wire thickness and $1 \mathrm{~mm}$ separation 


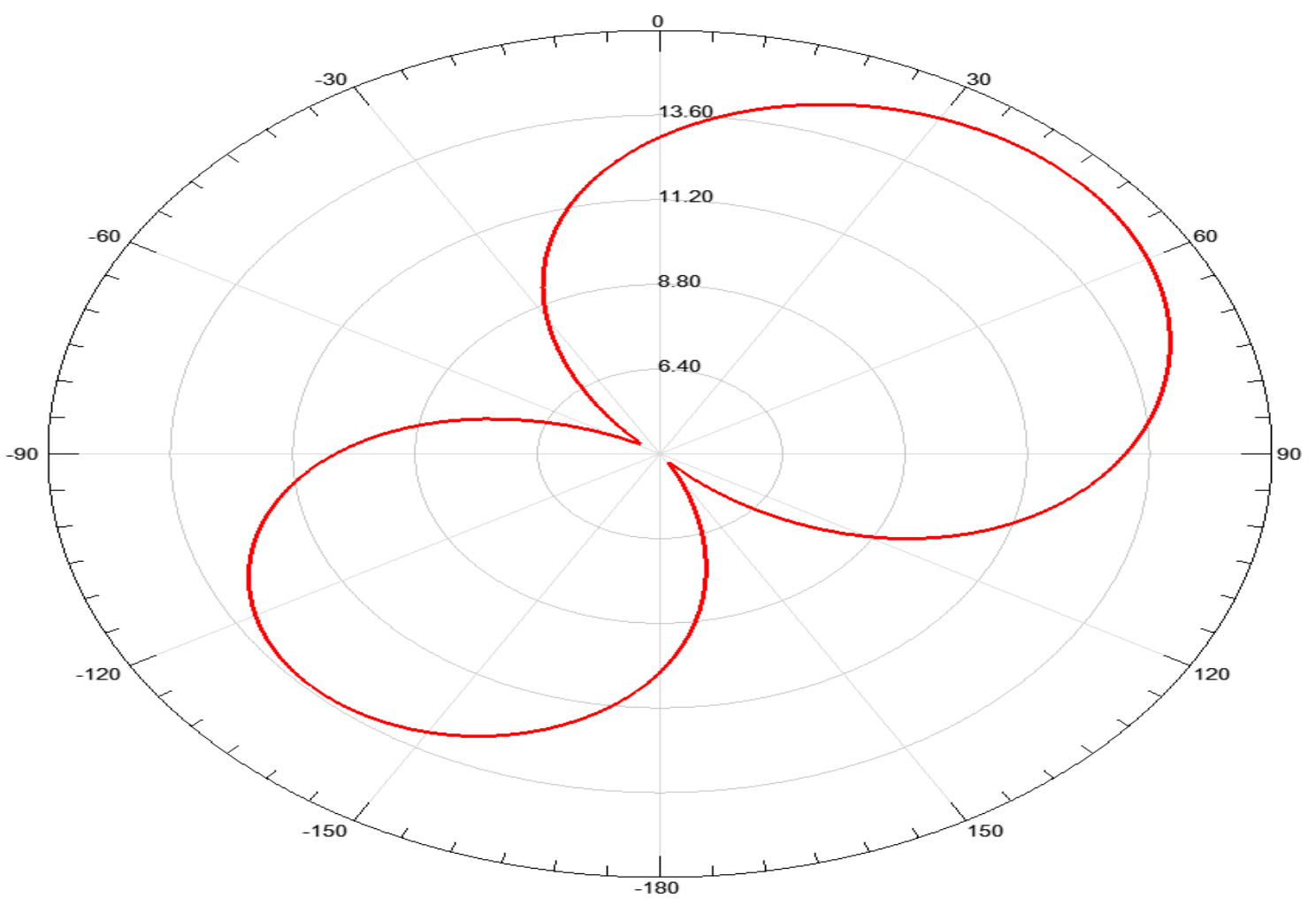

Figure 76: $2 \mathrm{~cm}$ leg length at $3.5 \mathrm{GHz}$ with $2 \mathrm{~mm}$ wire thickness and $1 \mathrm{~mm}$ separation

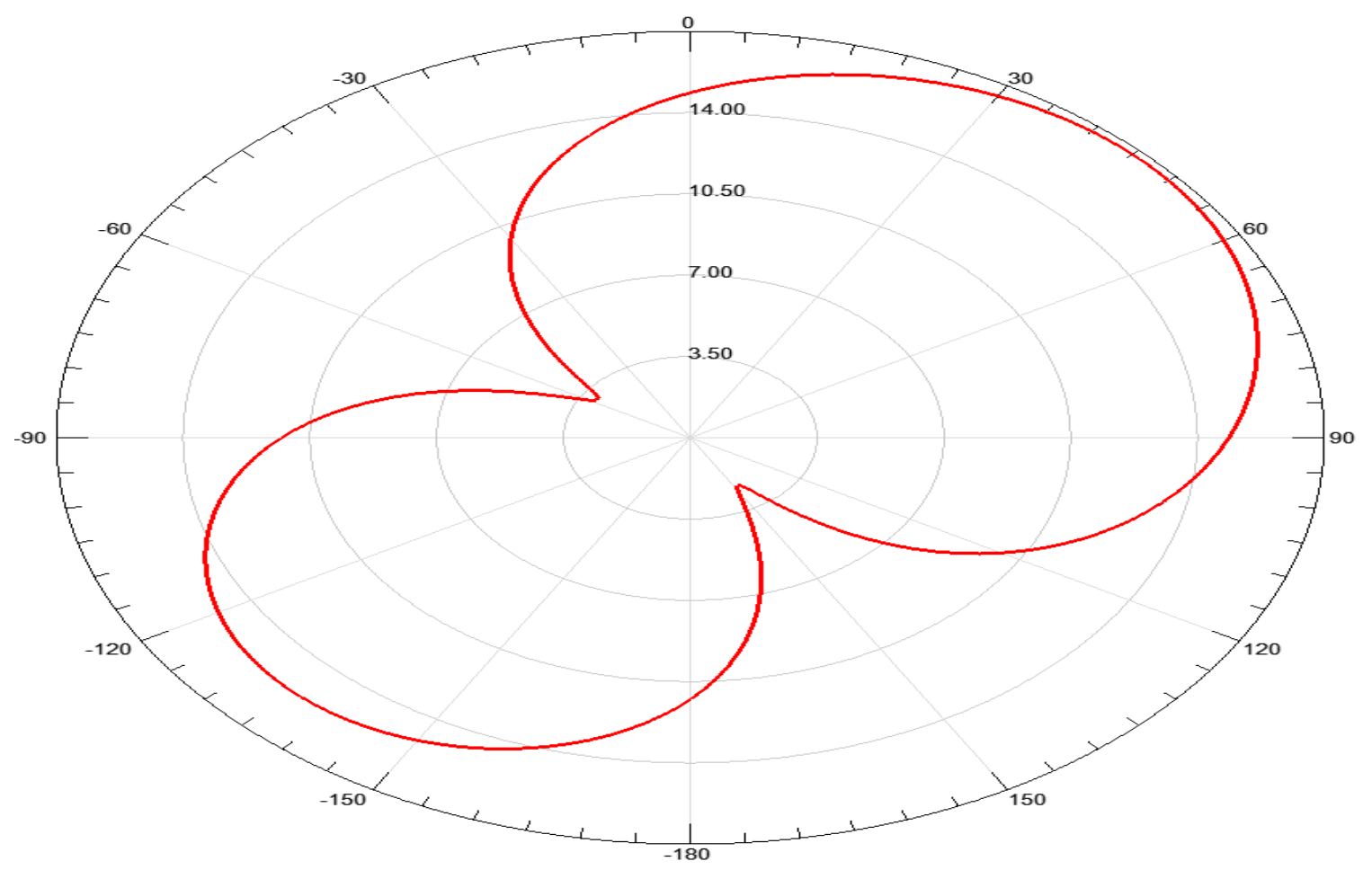

Figure 77: $2 \mathrm{~cm}$ leg length at $4 \mathrm{GHz}$ with $2 \mathrm{~mm}$ wire thickness and $1 \mathrm{~mm}$ separation 


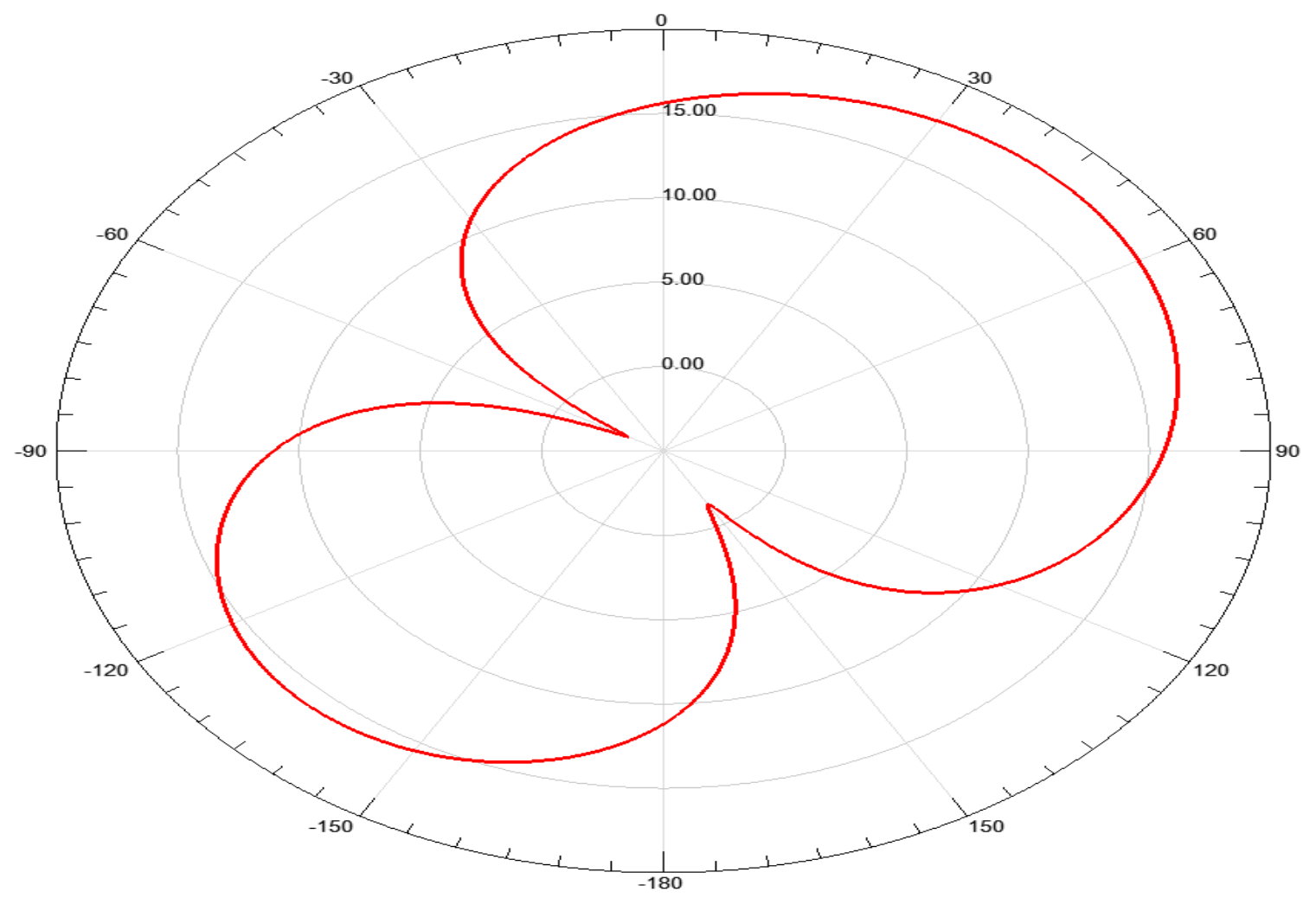

Figure 78: $2 \mathrm{~cm}$ leg length at $4.5 \mathrm{GHz}$ with $2 \mathrm{~mm}$ wire thickness and $1 \mathrm{~mm}$ separation

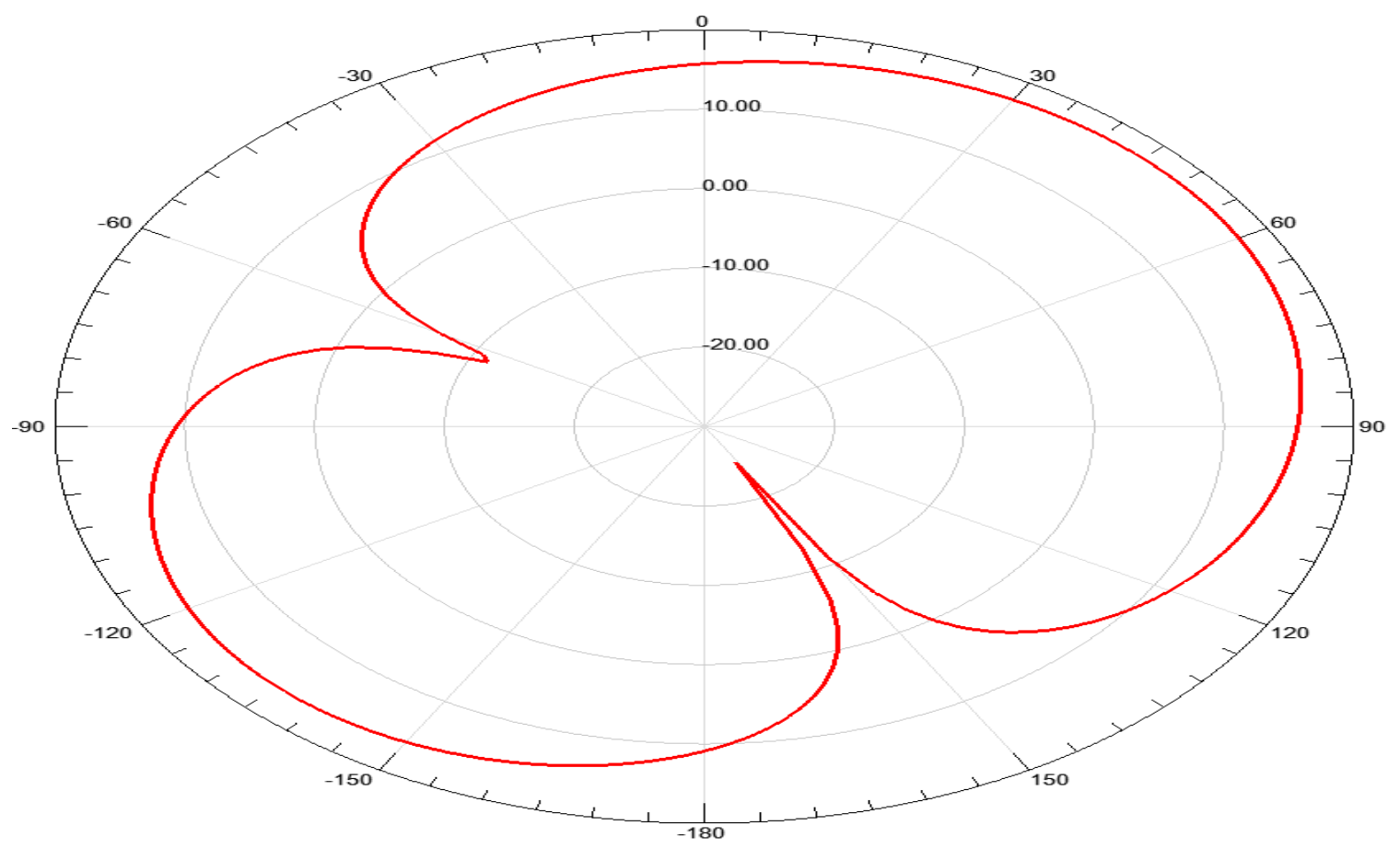

Figure 79: $2 \mathrm{~cm}$ leg length at $5 \mathrm{GHz}$ with $2 \mathrm{~mm}$ wire thickness and $1 \mathrm{~mm}$ separation 


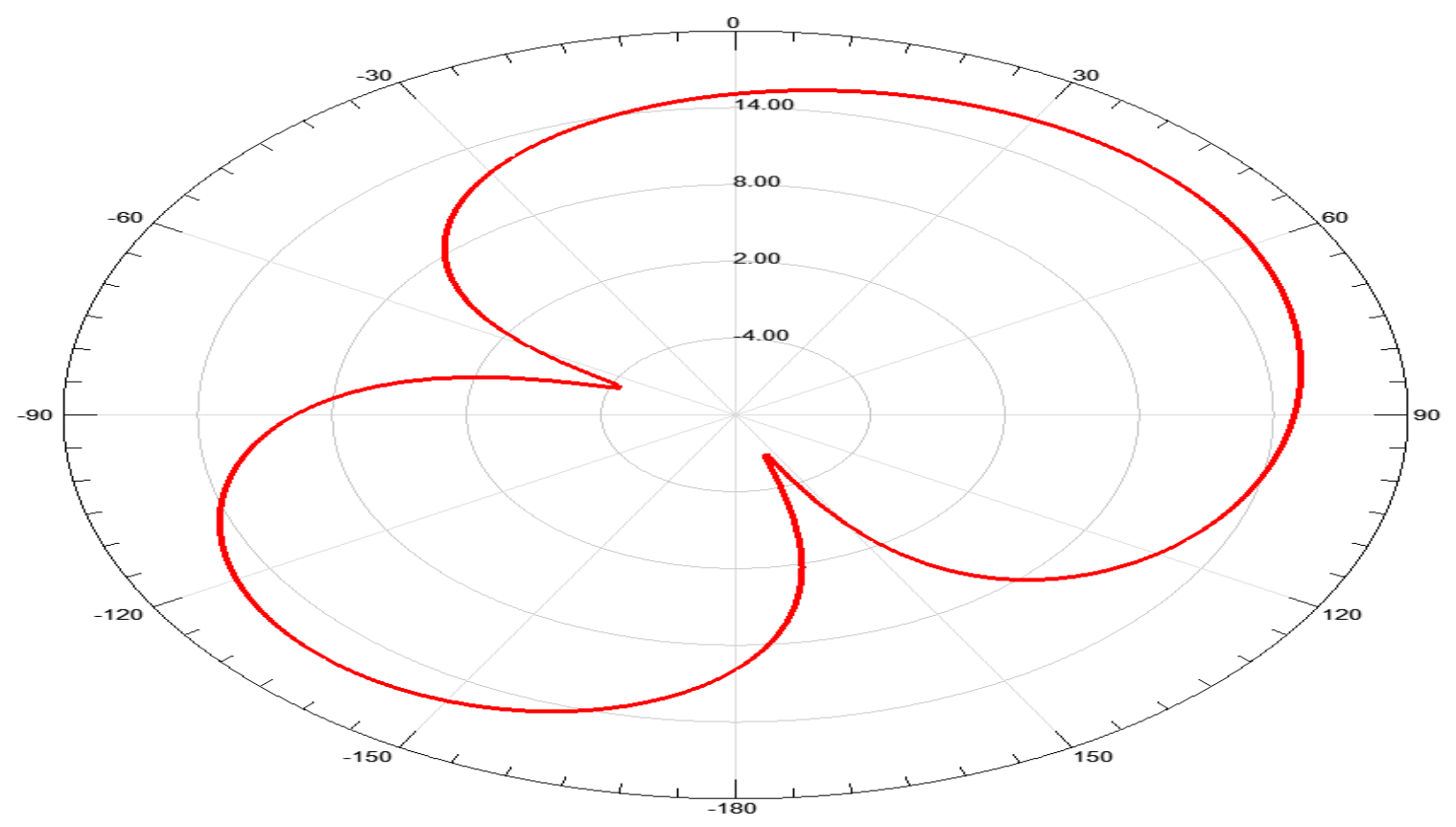

Figure 80: $2 \mathrm{~cm}$ leg length at $5.5 \mathrm{GHz}$ with $2 \mathrm{~mm}$ wire thickness and $1 \mathrm{~mm}$ separation

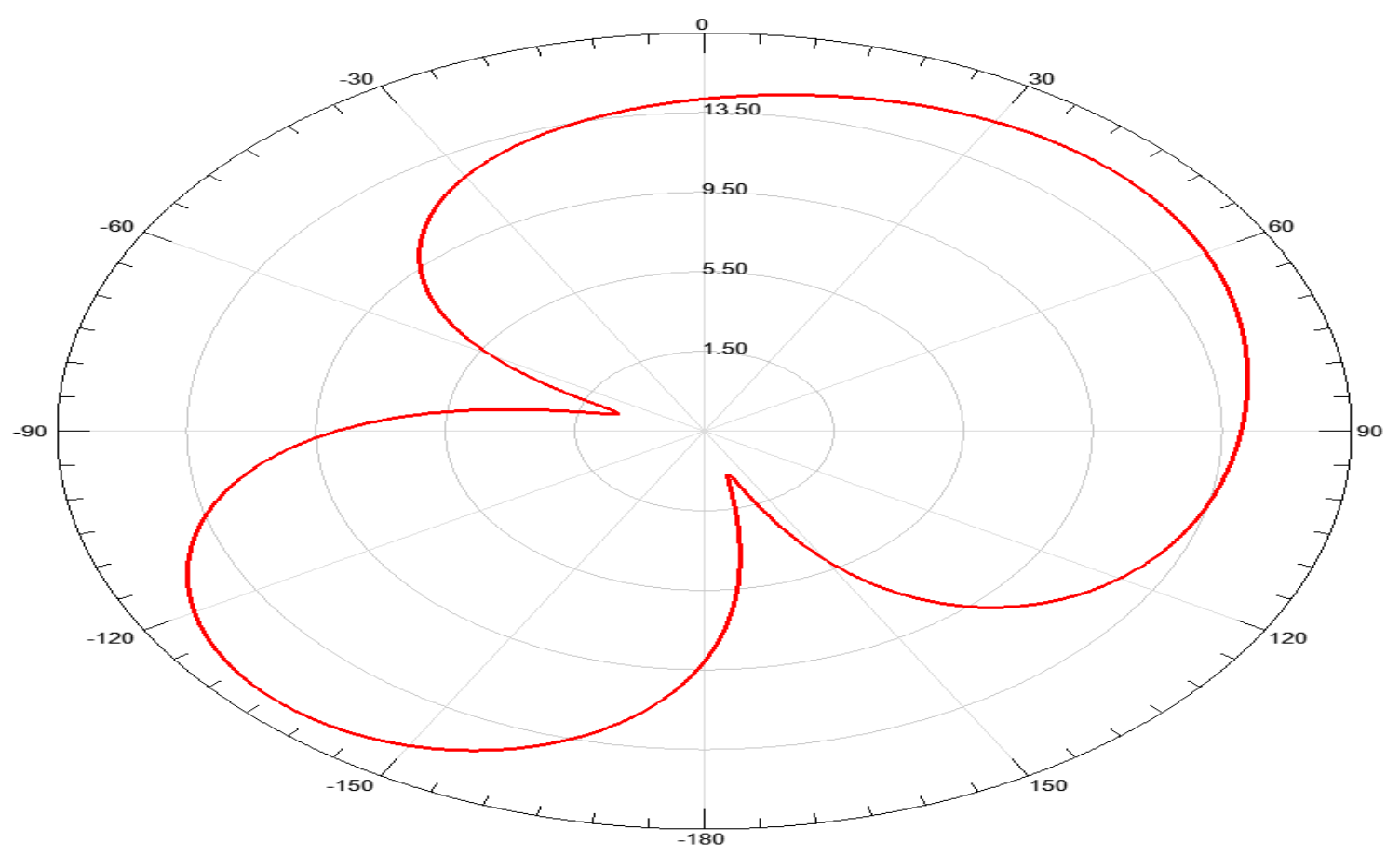

Figure 81: $2 \mathrm{~cm}$ leg length at $6 \mathrm{GHz}$ with $2 \mathrm{~mm}$ wire thickness and $1 \mathrm{~mm}$ separation 


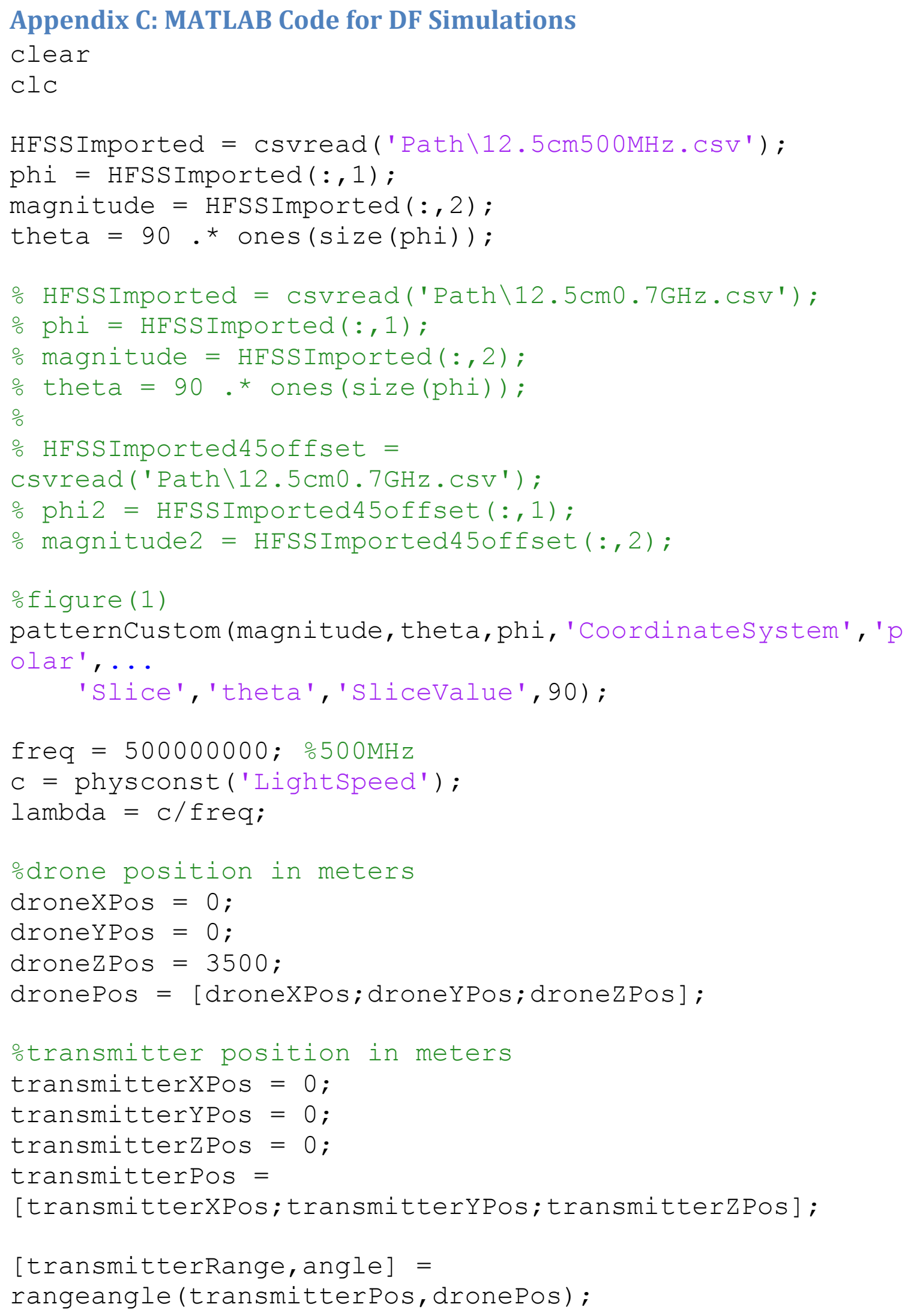




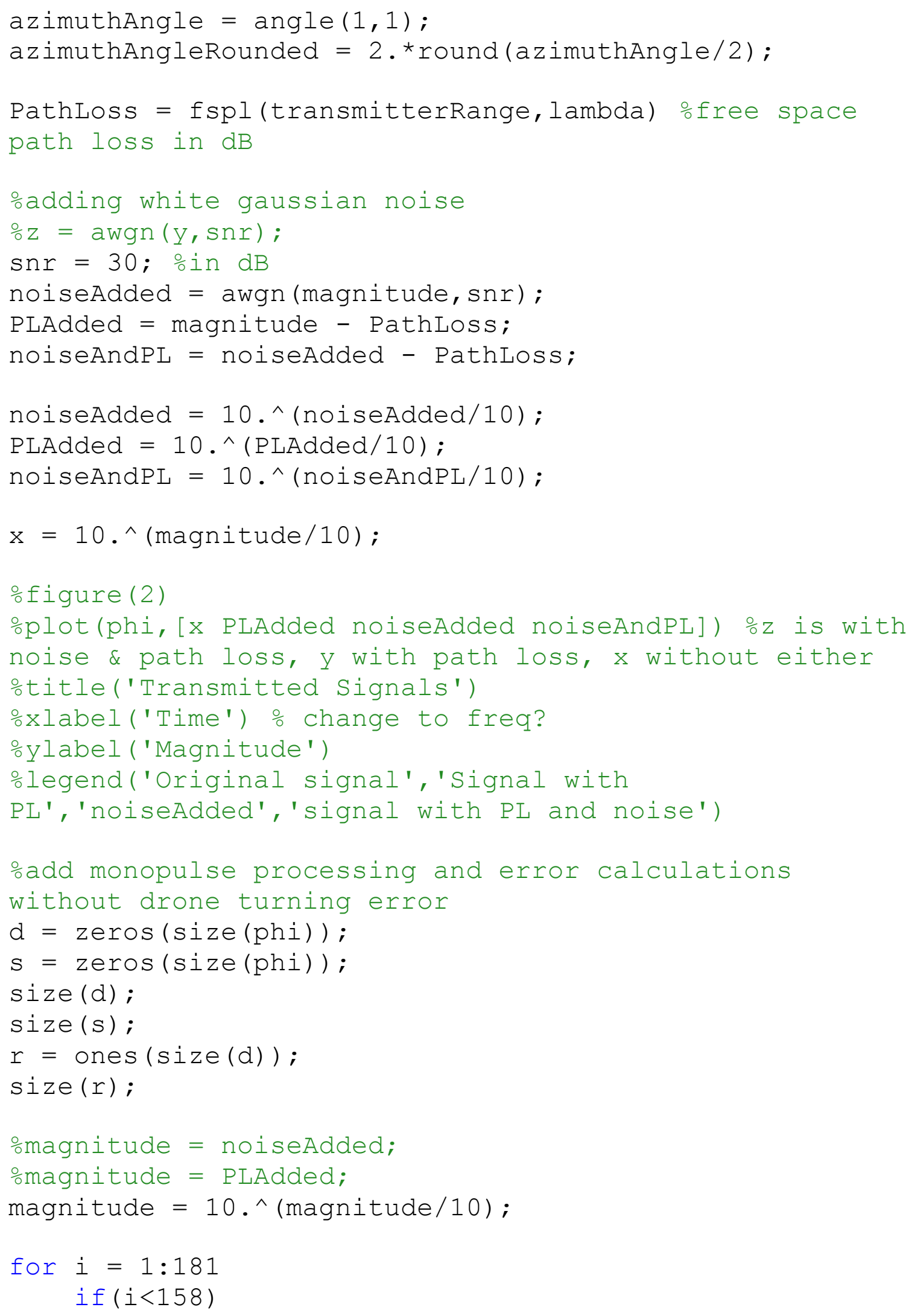




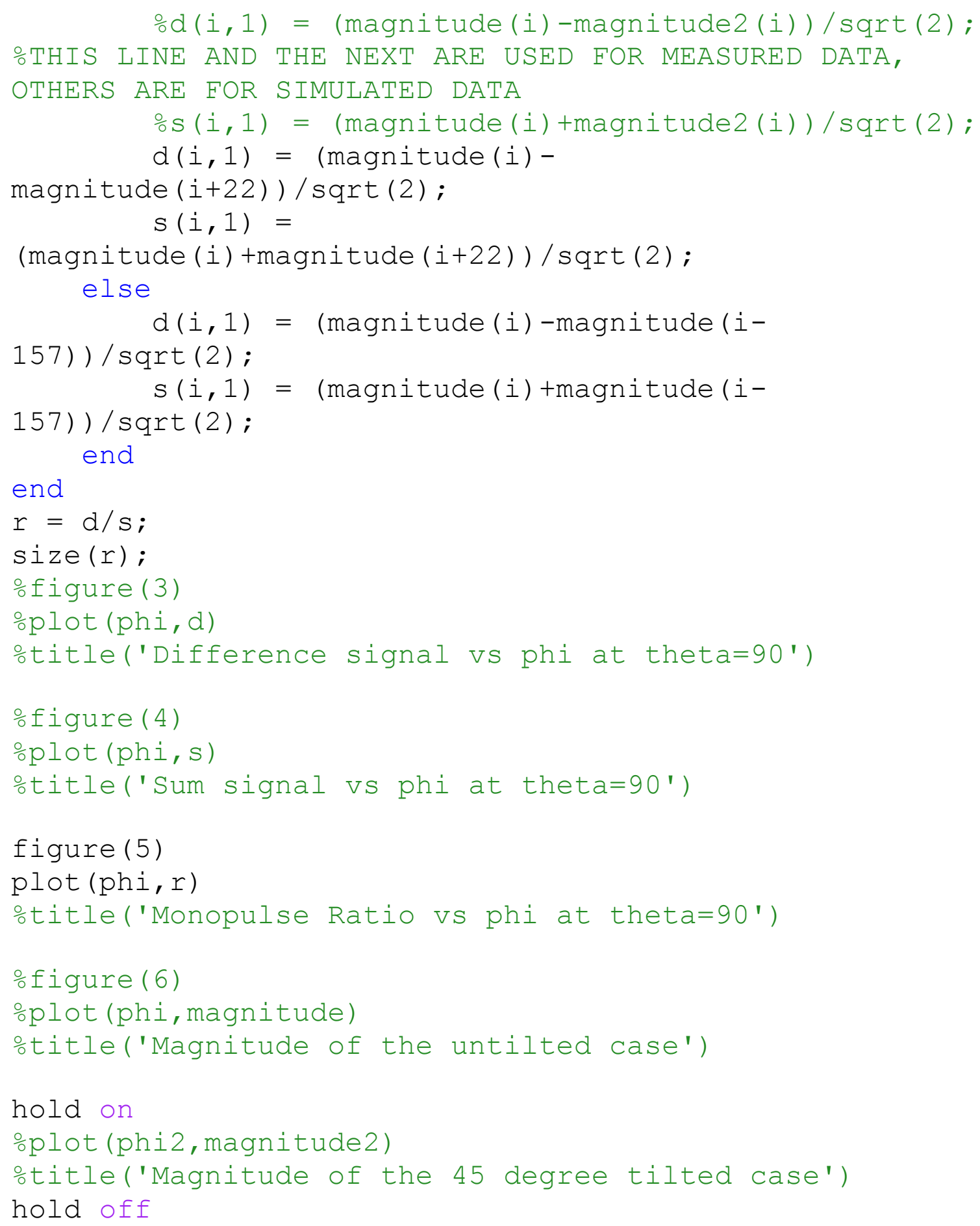

